Florida International University FIU Digital Commons

5-12-2015

\title{
The Microstructure and the Electrochemical Behavior of Cobalt Chromium Molybdenum Alloys from Retrieved Hip Implants
}

Christopher P. Emerson

cemer002@fiu.edu

DOI: $10.25148 /$ etd.FIDC000069

Follow this and additional works at: https:// digitalcommons.fiu.edu/etd

Part of the Biomaterials Commons, Manufacturing Commons, Metallurgy Commons, and the Orthopedics Commons

\section{Recommended Citation}

Emerson, Christopher P., "The Microstructure and the Electrochemical Behavior of Cobalt Chromium Molybdenum Alloys from Retrieved Hip Implants" (2015). FIU Electronic Theses and Dissertations. 2230.

https://digitalcommons.fiu.edu/etd/2230 


\title{
FLORIDA INTERNATIONAL UNIVERSITY
}

Miami, Florida

\section{THE MICROSTRUCTURE AND THE ELECTROCHEMICAL BEHAVIOR OF COBALT CHROMIUM MOLYBDENUM ALLOYS FROM RETRIEVED HIP IMPLANTS}

\author{
A thesis submitted in partial fulfillment of the \\ requirements for the degree of \\ MASTERS IN SCIENCE \\ in \\ MATERIAL SCIENCE AND ENGINEERING \\ by \\ Christopher Paul Emerson
}


To: Dean Amir Mirmiran

College of Engineering and Computing

This thesis, written by Christopher Paul Emerson, and entitled The Microstructure and the Electrochemical Behavior of Cobalt Chromium Molybdenum Alloys From Retrieved Hip Implants, having been approved in respect to style and intellectual content, is referred to you for judgment.

We have read this thesis and recommend that it be approved.

\author{
Anthony McGoron \\ Norman Munroe, Co-Major Professor \\ Kinzy Jones, Co-Major Professor
}

Date of Defense: May 12, 2015

The thesis of Christopher Paul Emerson is approved.

Dean Amir Mirmiran

College of Engineering and Computing

Dean Lakshmi N. Reddi

University Graduate School 


\section{DEDICATION}

I dedicate my master's thesis to my mother Alma Emerson, to my father Thomas Emerson, to my younger brother David Emerson and my Labrador Retriever, Luke. Their continued love and support has been priceless. 


\section{ACKNOWLEDGMENTS}

I wish to first thank the professors in the Mechanics and Materials Department who enriched my graduate education. Additionally, I am thankful for my major professor Dr. Kinzy Jones and co-major professor Dr. Norman Munroe for guiding me along the journey of my thesis dissertation. I would like to also thank committee member Dr. Anthony McGoron. There are three important individuals who have provided significant contribution in the laboratory aspect of my thesis. First and foremost, I am very thankful for the support of AMERI researcher, Dr. Alexander Franco. His training and guidance in metallurgical sample preparation and analytical analysis was valuable for the microstructural analysis of this project. Secondly, I am grateful for the support of current FIU PhD candidate Vishal Musaramthora. He provided valuable insight in conducting the corrosion experiments and analysis. Lastly, I am thankful for Mr. Jose L. Gonzalez for the creation of microstructure guide for hip prostheses that were given to the MME department at FIU for investigation. 
ABSTRACT OF THE THESIS

THE MICROSTRUCTURE AND THE ELECTROCHEMICAL BEHAVIOR OF

COBALT MOLYBENUM ALLOYS FROM RETRIEVED HIP IMPLANTS

\author{
by
}

Christopher Paul Emerson

Florida International University, 2015

Miami, Florida

\author{
Professor Kinzy Jones, Co-Major Professor \\ Norman Munroe, Co-Major Professor
}

Because of their excellent mechanical, tribological, and electrochemical properties, Cobalt Chromium Molybdenum alloys have been used as the material for both the stem and head of modular hip implants. Corrosion is one mechanism by which metal debris, from these implants, is generated, which can lead to adverse events that requires revision surgery. Manufacturing process such as wrought, as-cast, and powder metallurgy influences the microstructure, material properties, and performance of these implants

The current research focuses on analyzing the microstructure of CoCrMo alloys from retrieved hip implants with optical and scanning electron microscopy. Additionally, energy disperse spectroscopy was utilized to determine weight composition of cobalt, chromium, and molybdenum in solution. Potentiodynamic polarization was used as an accelerated corrosion testing method to determine the electrochemical behavior of the different microstructures. In agreement with prior literature, it was found that Low Carbon Wrought CoCrMo Alloys have the best corrosion resistance properties. 


\section{TABLE OF CONTENTS}

CHAPTER

PAGE

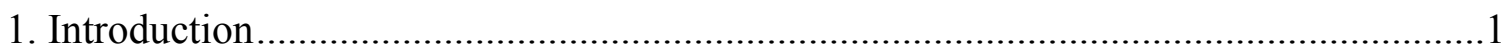

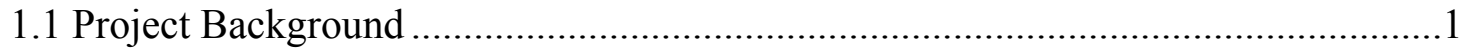

1.2 Summary of Research Goal..............................................................................

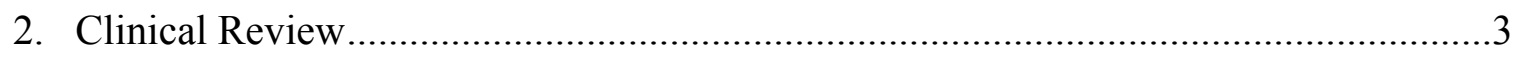

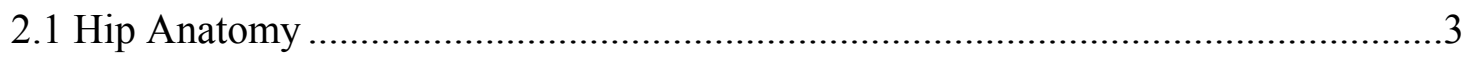

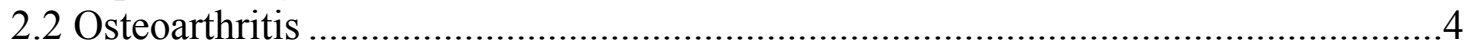

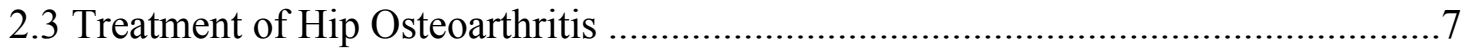

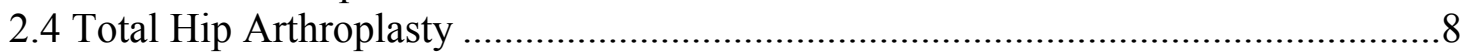

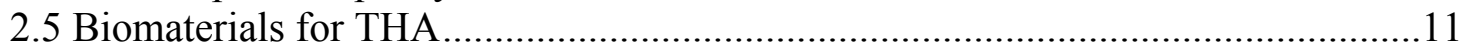

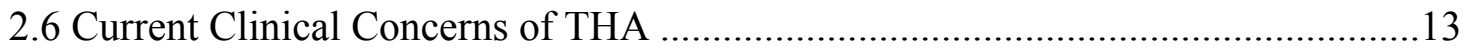

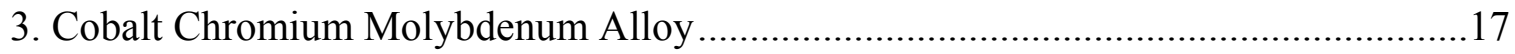

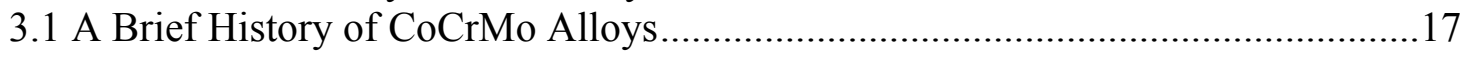

3.2 ASTM Requirements of CoCrMo Alloys ..........................................................18

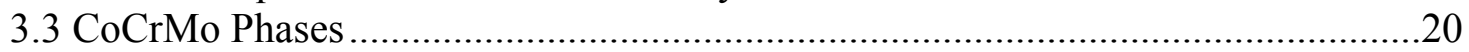

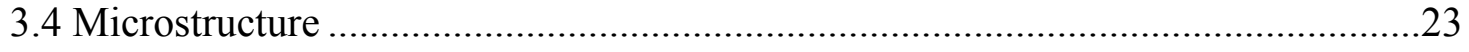

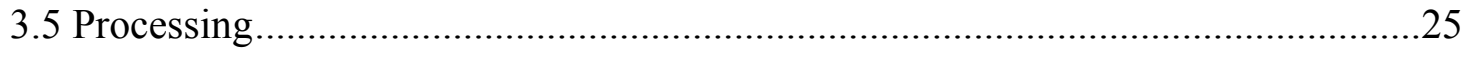

4. Electrochemical and Corrosion behavior of CoCrMo Alloys.........................................27

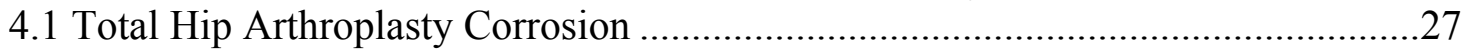

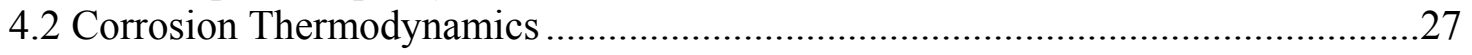

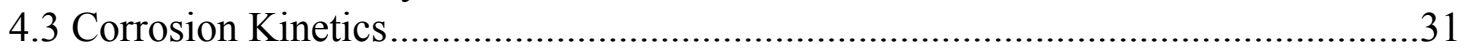

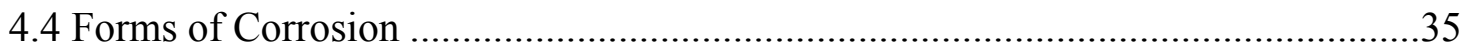

4.5 Corrosion of CoCrMo Implants …………………...........................................38

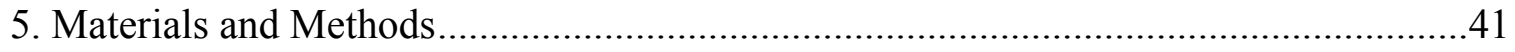

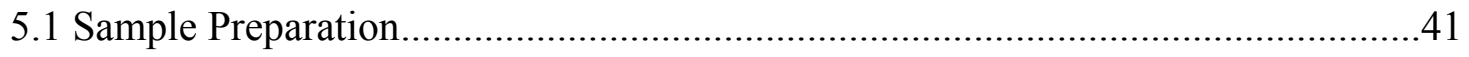

5.2 Corrosion Test Set-up..................................................................................43

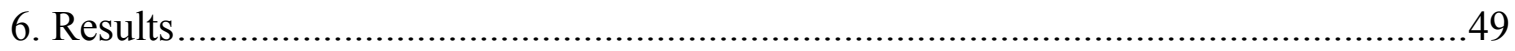

6.1 Microstructure Images Before and After Corrosion...............................................49

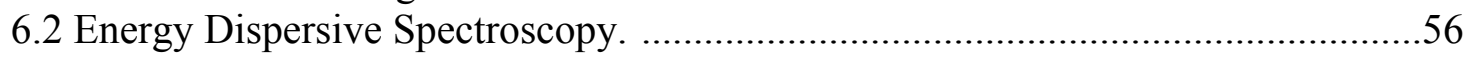

6.3 Potentiodynamic Polarization Graphs ...................................................................66

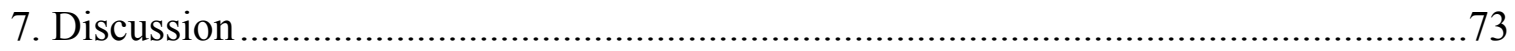

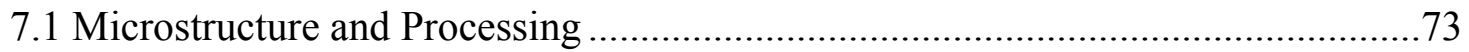

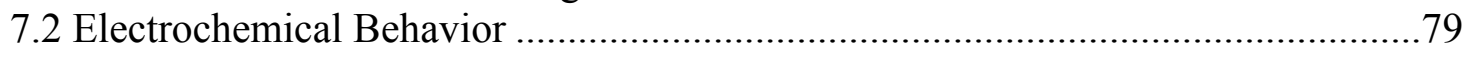

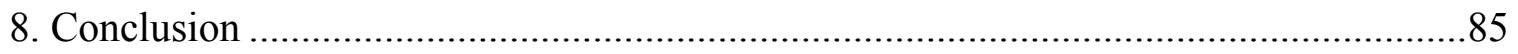




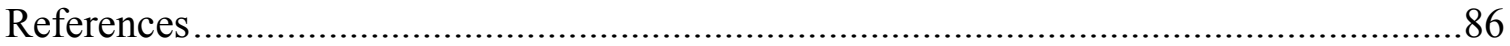




\section{LIST OF TABLES}

TABLE

PAGE

3.1 Chemical Composition of ASTM F75 and ASTM F-1537....................19

3.2 Mechanical Properties as reported in ASTM F75, F1537, and F799............19

5.1 Chemical Composition of Hanks Solution...................................45

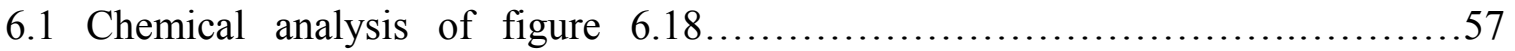

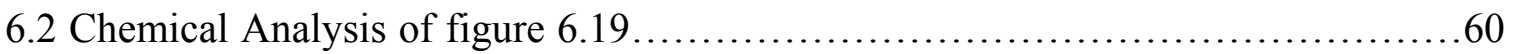

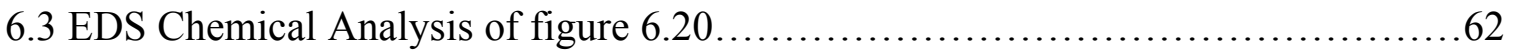

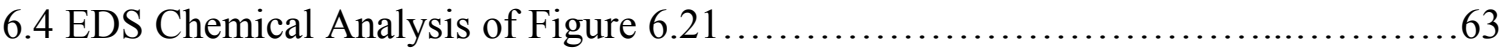

6.5 Chemical Composition of Samples.......................................65

6.6 in-Vitro Corrosion data of three different CoCrMo alloys........................71

7.1 Summary of in-Vitro electrochemical behavior of CoCrMo alloys..................84 


\section{LIST OF FIGURES}

FIGURE

PAGE

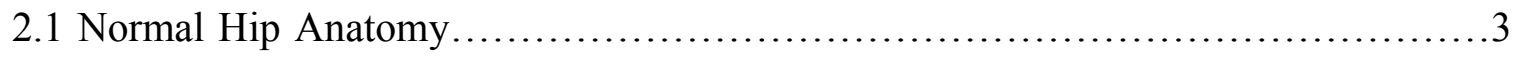

2.2 Radiograph of Hip Joint. ................................................

2.3 Hip Anatomy with Osteoarthritis.......................................6

2.4 Radiographic Image of Osteoarthritis Hip.................................6

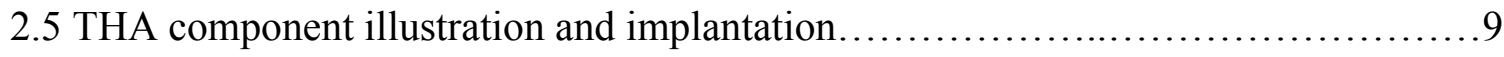

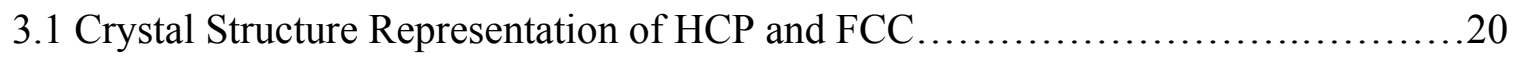

3.2 Co-Cr Binary Phase Diagram.......................................... 21

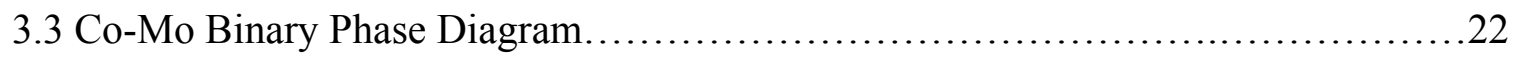

3.4 Typical As-Cast Microstructure..............................................23

3.5 Typical Wrought Microstructure..........................................23

3.6 Carbides and Phases in CoCrMo Microstructure................................25

5.1 Sample Sectioned from Explanted Hip Prosthesis............................42

5.2 Metal Sample Mounted in Epoxy...........................................43

5.3 Manual Grinding of Metal Sample........................................43

5.4 Assembly of Micro-corrosion Cell........................................44

5.5 Chemical Composition of Body Fluids....................................45

5.6 GAMRY Electrode Color Code..........................................46

5.7 Micro-corrosion cell, inside incubator, connected to the Potentiostat..............47

6.1 Polished Optical Micrograph of a Low Carbon Wrought Sample...................49

6.2 Corrosion Optical Micrograph of a Low Carbon Wrought Sample.................49 
6.3 Polished SEM Micrograph of a Low Carbon Wrought Sample...

6.4 Corrosion SEM Micrograph of a Low Carbon Wrought Sample......................50

6.5 Polished Optical Micrograph of an As-Cast Sample..............................51

6.6 Corrosion Optical Micrograph of an As-Cast Sample...........................51

6.7 Polished SEM Micrograph of an As-Cast Sample..................................52

6.8 Corrosion SEM Micrograph of an As-Cast Sample..............................52

6.9 Polished Optical Micrograph of a High Carbon Wrought Sample............................52

6.10 Corrosion Optical Micrograph of a High Carbon Wrought Sample........................52

6.11 Polished SEM Micrograph of a High Carbon Wrought Sample..............................53

6.12 Corrosion SEM Micrograph of a High Carbon Wrought Sample..........................53

6.13 Polished Optical Micrograph of a High Carbon Wrought

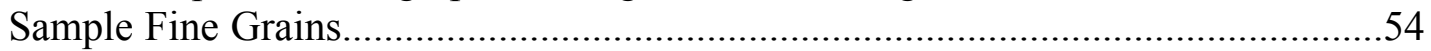

6.14 Corrosion Optical Micrograph of a High Carbon Wrought

Sample Fine Grains

6.15 Polished SEM Micrograph of a High Carbon Wrought

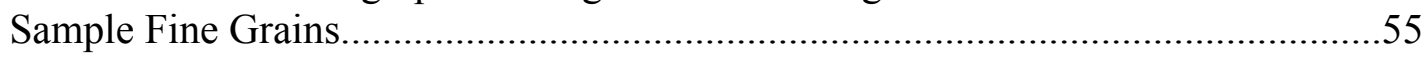

6.16 Corrosion SEM Micrograph of a High Carbon Wrought

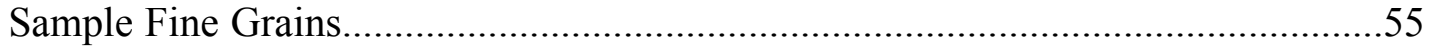

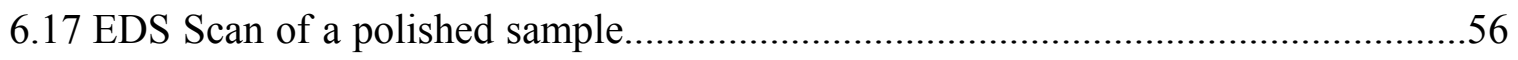

6.18 Representative EDS analysis of a polished sample.............................................57

6.19 EDS point scans of an As-Cast CoCrMo Alloy after corrosion.............................59

6.20 EDS Point Scans of High Carbon Wrought (fine grains) CoCrMo alloy after corrosion....................................................... 61

6.21 EDS Point Scans of a high carbon wrought (course grains) of a CoCrMo alloy after corrosion. 
6.22 EDS Analysis of White Secondary Phase.

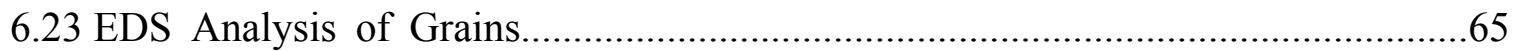

6.24 Typical potentiodynamic polarization graph for CoCrMo alloy................................66

6.25 Potentiodynamic Polarization Curve for Low Carbon Wrought

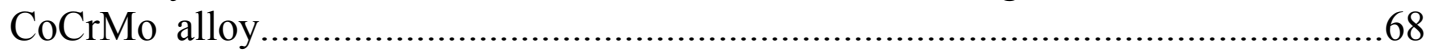

6.26 Potentiodynamic Polarization Curve for As-Cast CoCrMo Alloy............................69

6.27 Potentiodynamic Polarization Curve for High Carbon Wrought

CoCrMo Alloy..................................................................................................

6.28 Overlay of the electrochemical behavior of the low carbon wrought,

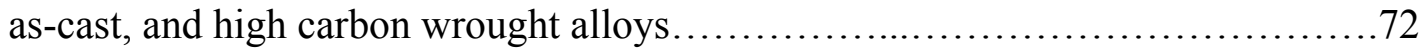

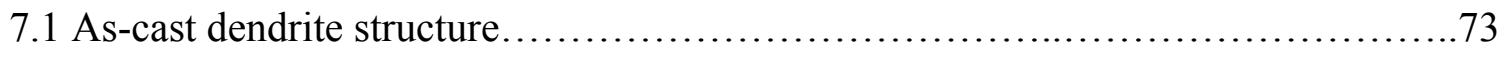

7.2 As-Cast CoCrMo microstructure unknown processing parameters....................74

7.3 As-Cast CoCrMo microstructure with 0.35 wt $\%$ carbon...........................74

7.4 Typical as-cast microstructure low magnification and

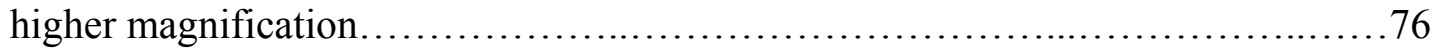

7.5 As-cast microstructure low magnification and higher magnification..................76

7.6 EDS analysis of as-received microstructure: Powder Metallurgy + Forging..........78

7.7 EDS analysis of as-received microstructure from hip prosthesis.....................79

$7.8 \mathrm{CoCrMo}$ electrochemical behavior........................................... 81

7.9 Inverted Overlay of potentiodynamic graphs from result section...................83 


\section{Chapter 1: Introduction}

\subsection{Project Background}

Orthopedic Surgeon, Carlos J. Lavernia MD, Medical Director and Chief of Orthopedics at the Center for Advanced Orthopedics at Larkin Community Hospital was granted an IRB to properly handle and sterilize hip implants that were removed from patients. Implants were properly sterilized, packaged, and ship to the Department of Mechanics and Materials at Florida International University to initiate a South Florida Adult Joint Reconstruction Retrieval Program. Furthermore, principle investigators Norman Munroe $\mathrm{PhD}$, and Kinzy Jones $\mathrm{PhD}$, developed an implant analysis proposal titled, "Evaluation of Explanted Prosthesis for Development of a Failure Analysis Protocol.” It was submitted to CEC Research Seed Grant 2011-2012. Beginning in 2013, master's degree candidate Jose L. Gonzalez began developing a metallurgical protocol to analyze mechanical and metallurgical variables of these failed implants that may have stimulated the need for revision surgery. His recently published dissertation "In-Vivo Corrosion and Fretting of Modular Ti-6Al-4V / Co-Cr-Mo Hip Prostheses: The Influence of Microstructure and Design Parameters" analyzed how different implant variables, both clinical and engineering, influenced the degradation of the Morse Taper between the femoral head and femoral stem of 48 hip implants. Mr. Gonzalez work is the first joint retrieval study conducted at Florida International University.

\subsection{Summary of Research Goals}

Corrosion and wear are two degradation methods of materials. For total hip arthroplasty one clinical concern is the issue surrounding tribocorrosion of loading bearing artificial joints. The goal of this thesis was to investigate the electrochemical behavior of cobalt 
chromium molybdenum hip implants utilizing in-Vitro accelerated potentiodynamic polarization tests. The processing parameters were unknown and there by metallurgical techniques were implanted to characterize the microstructures of the samples used in this investigation. Findings were compared to previously published studies. The investigations herein are part of the hip implant retrieval study. If the reader is left with one outcome it should be a clear understanding that different processing parameters can result different microstructures, which in turn, influence the material properties of an alloy - corrosion resistance. It is equally important to understand both the basic science and clinical science surrounding total hip arthroplasty. Thereby, an important aspect of this project was to provide a comprehensive review of the clinical aspects of total hip prostheses, a metallurgical review of CoCrMo alloys, and a review of electrochemistry/corrosion of this alloy in lieu of the clinical context. A final goal is to develop an in-Vitro corrosion analysis protocol to provide complimentary data to the hip retrieval protocol. Thereby future investigators will be able to use this study as a guide to conduct corrosion analysis on CoCrMo implants. 


\section{Chapter 2: Clinical Review}

\subsection{Hip Anatomy}

The hip joint provides excellent stability and range of motion between the lower extremities and the trunk. This joint can be viewed as a ball-in-socket rotating mechanism. The acetabulum, located within the pelvis, is a cup shaped cavity where the head of the femur sits. Additionally, the acetabulum is the junction of three pelvic bones: the illium, the ischium, and the pubis [1]. The head of the femur (femoral head) is at about $130^{\circ}$ to the axis of the femoral shaft in the coronal plane [2]. A cartilage layer protects the joints, and the synovial fluid acts as a natural lubricant to minimize wear between the rotating features. All humans have the same anatomic features on the hip joint; however, the size and morphology can greatly vary [3]. Figure 2.1 is a simplified illustration of the anatomy of the hip, while figure 2.2 is a radiograph of the hip joint.

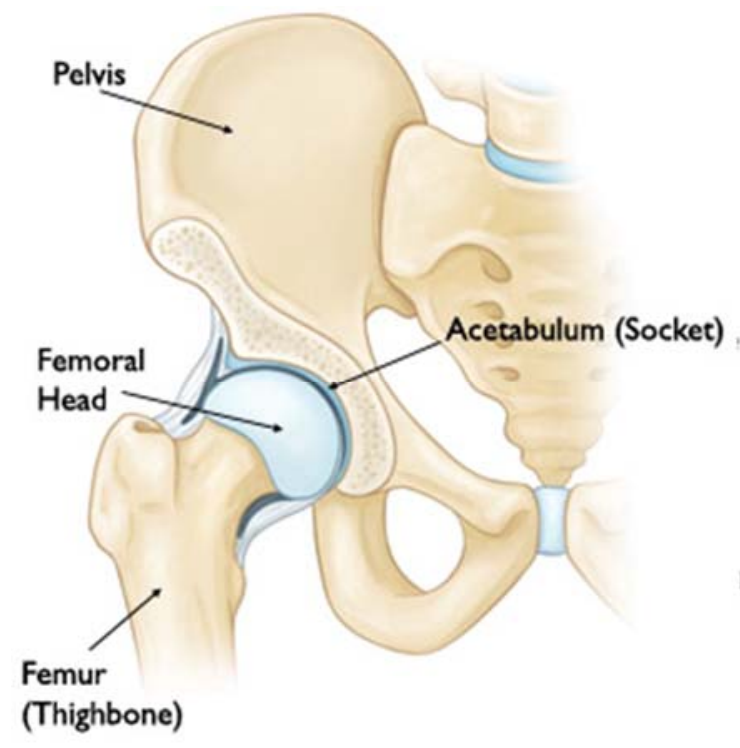

Figure 2.1: Normal Hip Anatomy [4]

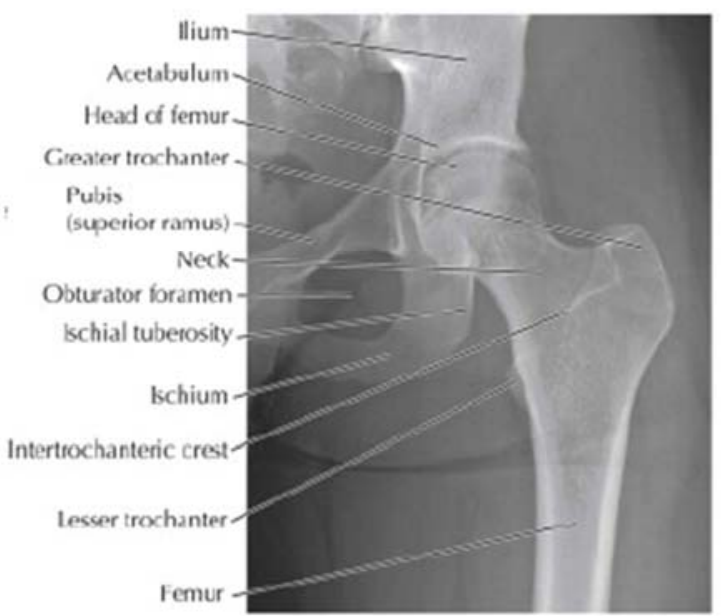

Figure 2.2: Radiograph of Hip Joint [5] 
In order to maintain healthy load-bearing joints, such as the hip, mechanical loading plays an important role in stimulating osteocytes (bone cells) to constantly maintain healthy bone tissue. Osteoblasts produce the proteins and transport bone mineral (hydroxyapatite) to form the tissue matrix known as bone. Meanwhile, osteoclasts break down bone. Thus old bone is replace with new bone. In joints, cartilage helps protect the underlying bone from wearing out during gait cycles. Cartilage is composed of chondrocytes (cartilage cells). Studies have investigated how these cells respond to forces and mechanical stresses [6]. Chondrocytes are composed of type II collagen and aggrecan (proteoglycan aggregates). Collagen provides the tensile strength, while aggrecan provides the stiffness of the cartilage [7]. Moderate cyclic stimuli are necessary to regulate the metabolic process of chondrocytes and play an important role in cartilage homeostasis. Excessive mechanical loading can lead to microdamage in the cartilage matrix leading to chondrocyte fatigue failure and induced tissue damage [8]. It is the damage of the cartilage that leads to the progression of joint diseases such as osteoarthritis.

\subsection{Osteoarthritis}

Osteoarthritis (OA), a cartilage degrading joint disease, is a primary source of physical disability amongst the elderly population. This disease imparts over $\$ 60$ billion in healthcare costs and it is estimated that $25 \%$ of the US population with be effected by this disease. The risk factors associated with OA include genetic predisposition and aging, among others; however, the exact pathogenesis of this disease remains unknown [9]. Currently, it is estimated that some 27 million individuals in the United States are afflicted with OA [10]. Investigations are advancing to better understand the molecular 
mechanisms of this disease in addition to the development of novel biological therapies to impede or reverse cartilage degradation [11]. Cartilage is composed of chondrocytes, which are responsible for maintaining healthy synthesis and degradation cycles of the extracellular matrix (ECM) components (collagens and proteoglycans) of the articular cartilage. A disruption between the dynamic equilibrium of these two processes can led to high oxidative states leading to apoptosis of cells and cartilage loss. Degradation of the cartilage ECM manifests itself in clinical settings as arthritic pain and physical disability [12]. A lack of preventative measures leads to worsen functional disability. Cartilage and synovial injury influences the peripheral receptors surrounding the joint to produce the sensation of pain [13]. Figure 2.3 is a simplified illustration of cartilage degraded at the hip joint, while figure 2.4 provides a radiograph. The clear difference between figure 2.3 and 2.4 is that the joint cavity between the pelvis and head of the femur is narrowed in an osteoarthritic hip joint. 

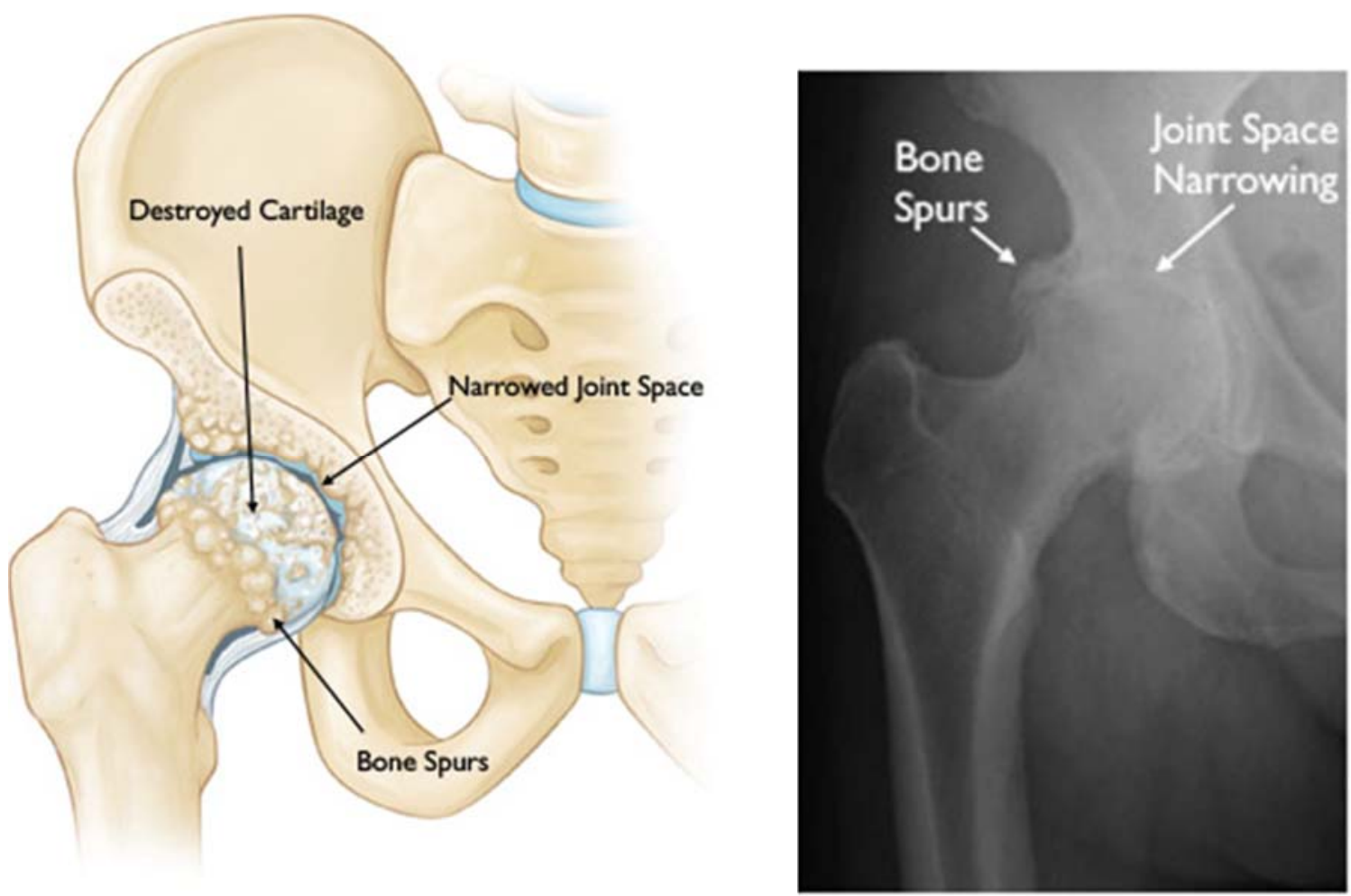

Figure 2.3: Hip Anatomy with Osteoarthritis (left) [4]

Figure 2.4: Radiographic Image of Osteoarthritis Hip (right) [4]

Inflammatory stimuli from cytokines (interleukin-1, interleukin-6, and interleukin-17), tumor necrosis factor- $\alpha$ (TNF- $\alpha$ ), and prostaglandins (PGE2) play a role in stimulating cartilage-degrading proteases to initial ECM degradation, while also contributing to neurological pain pathways associated with OA. A few interesting biological compounds have been well established to inhibit these catabolic mediators. The inflammatory inhibitors include resveratrol (RSV) and bovine lactoferricin (LfcinB). Resveratrol is a natural polyphenol with anti-inflammatory, anti-oxidant, cardio-protective, and antitumor properties. It is responsible for the health benefits of moderate red wine consumption [14]. Lactoferricin is a 25 amino acid peptide found in cow's milk with anti: 
inflammatory, viral, bacterial, oxidant, pain, and tumor properties. [15]. Studies of both RSV and LfcinB for the treatment of OA are underway [11]. Although progress has been made in better understand the molecular pathways of osteoarthritis - along with mechanisms found to disrupt the degradation process, treatment options for individuals suffering from severe osteoarthritis are quite limited.

\subsection{Treatment of Hip Osteoarthritis}

Many clinical guidelines for the treatment of OA have been established and are generally accepted; however, excellent clinical implementation is lacking [16]. Currently, there are five available treatment options aside from pharmacological interventions, as reported by Nelson et. al., namely: 1) education and self management, 2) exercise and weight lose, 3) assistive devices, 4) alternative and complementary approaches, and 5) surgical intervention [17]. In regards to individuals with hip OA, a consensus amongst guidelines includes individualized treatment plans for self care, low-impact aerobic exercises (land or water based), and walking aids (canes and walkers) [18]. Because of the slow deterioration of the joint, which will cause an increase in pain and loss of function, the duration of pain relief and improvement in function varies. Pharmacologic agents include oral administration of acetaminophen, non-steroidal anti-inflammatory agents, neutraceuticals, and corticosteroid injections [19]. Injections of viscosupplements such as hyaluronic acid has been demonstrated for efficacy and is used in both Europe and the United States for treating knee OA; however, there is no FDA approval for utilizing hyaluronic acid to treat hip OA. A meta-analysis by Lieberman et al., has indicated that the use of hyaluronic acid to treat hip OA showed a slight improvement over controls with statistical significance; however, multicenter retrieval studies are needed for true 
efficacy of hyaluronic injections to help decrease pain in patients with hip OA [19]. Additionally, for patients who qualify, hip joint replacement is recommended. Joint replacement for osteoarthritis of the hip is the only intervention that has been successful for many years; yet it is only suitable for patients with advance osteoarthritis or when other treatment options to mitigate pain have been unsuccessful [20].

\subsection{Total Hip Arthroplasty}

Total hip arthroplasty (THA) is a joint replacement technique whereby the head of the femoral stem is sectioned off, the femoral canal is reamed, and the acetabulum is shaved to make way for an artificial hip prosthesis. THA devices can be categorized in three groups: stem fixation, taper modularity, and bearing interfaces. Stem fixation was originally achieved through the bone cement known as polymethylmethacrylate (PMMA). Improvements in stem technology began to utilize osteointegration into the implant by using a press-fit stem, with porous proximal surface and possibly coated with hydroxyapatite to stimulate bone in-growth. Original THA devices were monoblock, meaning the head and stem were one solid metal component. Modularity was successfully incorporated into the 1980 s to allow the stem and head to be made of separate materials. The rationale was that the femoral head needs excellent wear resistance, while the stem should have a modulus of elasticity similar to bone. By the 1990s, implants were being developed with dual modular tapers meaning a middle component, known as the neck, separated the stem from the head. Furthermore, THA prostheses can be divided into Hard-on-Soft (HoS) and Hard-on-Hard (HoH) constructs. HoS bearing groups can be either Metal-on-Polyethylene (MoP) or Ceramic-onPolyethylene (MoP), while $\mathrm{HoH}$ can be Metal-on-Metal (MoM), Metal-on-Ceramic 
$(\mathrm{MoP})$, or Ceramic-on-Ceramic $(\mathrm{CoC})$. Figure 2.5 provides an illustration of each component of the THA device (left), a fully assembled device (center), and a representation of the THA device inside the femur and connected to the pelvis (right).

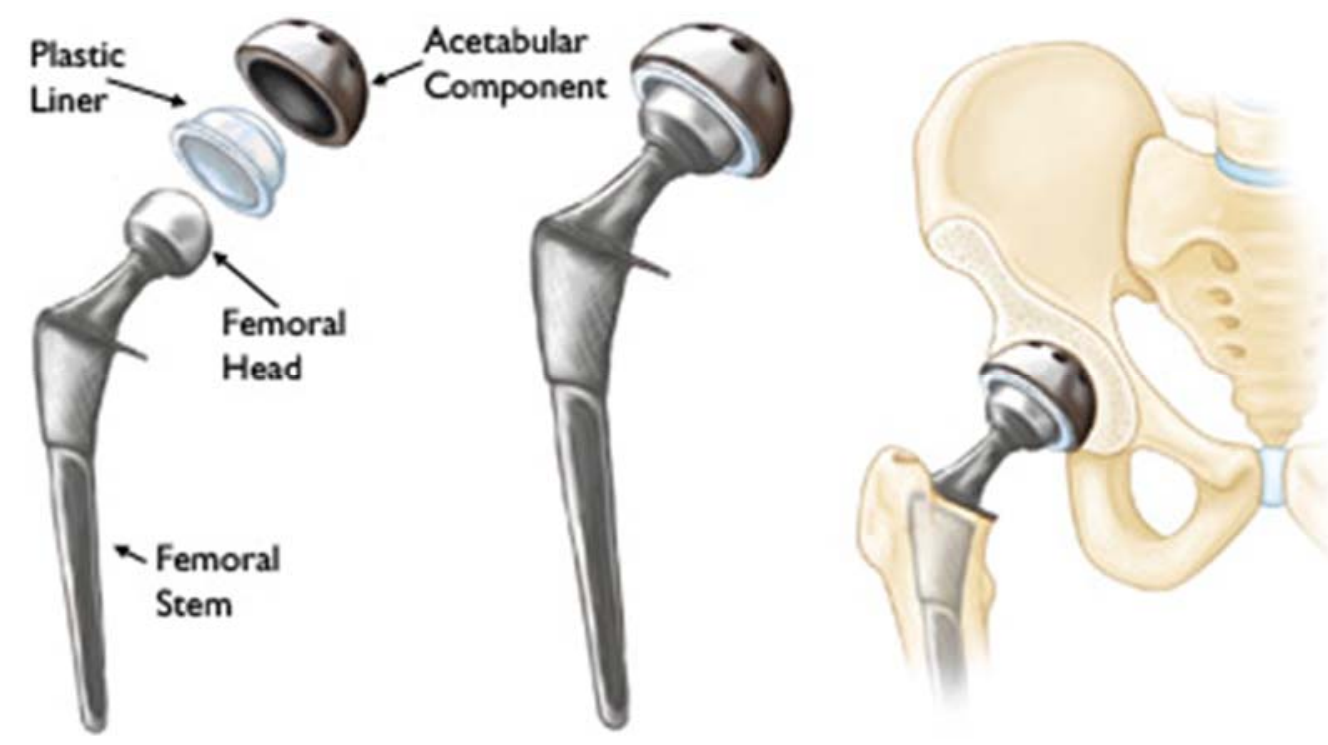

Figure 2.5: THA component illustration and implantation [4]

In the early 1960s, the two main monoblock implants for THA were the Charnley Stem (DePuy, Leeds, UK) and Exeter Hip (Howmedica International, London, UK). Both systems provided excellent long-term clinical outcomes [21]. The early 1960s, began the launch of cemented stem fixation in hip prosthesis [22]. In the 1960s, Charnley chose PMMA bone cement to provide mechanical fixation of the acetabulum in his low-friction THA design and it remained the standard for 20 years. Ten-year follow-ups provided excellent results; however increasing radiographic signs of loosening began emerging at 20-year follow-ups. Investigators believed that elimination of PMMA bone cement would 
eliminate asceptic loosening and increased implant longevity [23]. In 1977, the introduction of cementless fixation became available in the United States, with the hope to provide better long-term fixation. In the early 1980s, femoral head-neck modularity became increasing popular because intraoperative adjustments of limb length and offset, and different combination of head and stem became available [24]. Implant design and processing are highly varied from each manufacturer. Modularity allowed combination of a less rigid titanium alloy stems to mate with a more wear resistant cobalt alloy heads through the use of a Morse taper. The Morse taper has a male component on the femoral stem known as the trunnion and a female component on the femoral head known as the bore. Tapers for each construct may very in regards to their proximal diameter, distal diameter, taper length, included angle, manufacturing tolerances, and surface finish, and surface treatment. These factors may influence the degradation of the taper upon retrieval analysis [25]. Impingement may occur when two nonarticular surfaces come into contact during joint range of motion (ROM), creating torque that can lead to dislocation. The advantages of using larger diameter heads includes: a higher head to neck ratio that reduces component impingement, increasing range of motion, and since larger heads sit deeper within the acetabular liner, the risk of dislocation is reduced [25]. More recently, Langton et al., identified three design changes of large head MoM hips: altered trunnion dimensions, altered trunnion surfaces, and trend of increase head diameter [23]. The trunnion is the male taper at the proximal end of the femoral stem that connects to the femoral head. In general, the trunnion has been slimmed down from a 1416 to the 12/14 (proximal/distal diameter of taper measured in millimeters). Trunnions are being manufactured with ridged surfaces to create one stem for multiple bearing surfaces, such 
as ceramic heads. Furthermore, femoral head size has increased from the original $22 \mathrm{~mm}$ head to heads as large as $52 \mathrm{~mm}$ [23]. All these variables have been areas of investigation in regards to clinical concerns that may cause adverse effects in patients with hip prostheses.

\subsection{Biomaterials for THA}

Materials fall into three broad categories: metals, polymers, and ceramics. Biomaterial is the classification of the successful extension of a material that is used to replace and/or restore physiological function. Biomaterials are implantable materials in biological systems that have a synergistic response with the host environment. The chemical and physical properties, as well as the surrounding environment will determine the in vivo biological stability of implantable materials [26]. Metallic biomaterials fall into three groups of metallic elements, which include cobalt based, iron based, and titanium based alloys. They have been used as orthopedic implants (screws, pins, and plates), bone fixators (internal and external), and joint replacement devices. Such materials can be used to help accelerate fracture healing and replace function of hard tissues because of their rigidity, fatigue strength and fracture toughness [27]. One of the most important material properties of orthopedic implant metals, such as cobalt, titanium, and stainless steel based alloys, is their corrosion resistance. These materials form a thin passive oxide surface that drastically reduces the rate of metal ion dissolution by many orders of magnitude. The clinical use of ceramic materials as a bearing surface began in the early 1970s. In 1982, Smith and Nephew's Mittelmeier Autophor began the first FDA approved ceramic material, aluminum oxide (alumina or $\mathrm{Al}_{2} \mathrm{O}_{3}$ ), for clinical use in joint replacement. Ceramic materials provide excellent biocompatibility and surface properties for bearing 
applications, when compared to metals, because they are significantly harder and form smoother surfaces [28]. This leads to better tribological properties. Additionally ceramics, such as alumina oxide, are bio-inert. Early reports of failure associated with ceramic materials included lack of fracture toughness, leading to ceramic implant bursting. The longevity of ceramic material was questioned [29]. The quality of alumina ceramic heads has significantly improved over the last 40 years. Improvements in manufacturing processes have been able to enhance mechanical strength and fracture toughness of these materials through solidification of denser ceramics and grain boundary reduction of the material [30]. Although there have been studies with ceramic-on-ceramic as articulating surfaces, issues with squeaking has limited their intended use; however, newer alumina material has been successfully used as the material of choice for the femoral head component. Ceramtec markets their alumina heads under the registered name Biolox. It currently represents $100 \%$ market share for ceramic femoral heads and is designed to be compatible with the stems of the other manufacturers. Ceramic femoral heads present a new alternative to the more traditional cobalt chromium alloyed heads. Ceramic heads offer excellent mechanical integrity in compression and bioinert particle debris without any reported adverse effect. Polymers have been used as a bearing surface for the liner of the hip replacement devices. British orthopedic surgeon, Sir John Charnley, was the first to incorporate ultrahigh molecular weight polyethylene (UHMWPE) in hip replacement devices. Compared to other polymers, UHMWPE has excellent impact resistance and tensile strength. It is also one of the more wear resistant plastics. UHMWPE has undergone several manufacturing and sterilization updates since the 1990s [31]. Gamma irradiation can cause polymer chain excision and oxidation, 
leading to reduction in mechanical properties. By the late 1990 s, other sterilization techniques, such as ethylene oxide, have been utilized because of the reduced amount of polymer degradation during the sterilization process. Careful dosage of radiation can cause UHMWPE to form crosslinks. If formed properly crosslinking the fibers of UHMWPE will greatly improve the polymer's wear resistance [32]. More recently, vitamin $\mathrm{E}$ has been added to the crosslinked UHMWPE as an antioxidant to reduce the formation of free radicals during processing and to decrease in-Vivo oxidation. This second generation of crosslinked UHMWPE infused with Vitamin E has been successful implanted as the lining material in hip since 2007. The incorporation of metal, ceramic, and plastic as part of the hip prosthesis has been successful.

\subsection{Current Clinical Concerns of THA}

Although THA has had over 40 years of clinical success, risks of adverse effects remain. One of the first clinical concerns results from the wear of UHMWPE. Polymer debris generated from the wear of UHMWPE (from HoS constructs) has shown to cause osteolysis. To mitigate this issue, $\mathrm{HoH}$ constructs were developed. The FDA approved second generations MoM constructs in 2002, first generation CoC constructs in 2003, and first generation MoC constructs in 2011. By the mid-2000s, nearly 50\% of primary THA patients received $\mathrm{HoH}$ hip prostheses [33]. Berry et al., identified four general mechanisms of degradation of the hip implant that are of current clinical importance, which include polyethylene wear, ceramic-on-ceramic $(\mathrm{CoC})$ bearing wear, metal-onmetal (MoM) bearing wear, and taper tribocorrosion [34]. Concerns with polyethylene wear originate from the result of polymers generally having lower wear resistances as compared to metals and ceramics. Polyethylene wear would result in polymer debris that 
could lead to adverse local tissue reactions. The creation of highly crosslinked polyethylene (HXLPE) has significantly reduced the wear rates of the plastic liner [35]. Hard-on-hard bearings $(\mathrm{HoH})$ such as $\mathrm{CoC}$ and $\mathrm{MoM}$, reported significant better tribology performance and less wear debris in hip simulator studies, as compared to Metal-on-Polyethylene (MoP). Their performance in the laboratory promise, yet clinical results were far from ideal. Ceramics are the hardest and, generally speaking, the material class with some of the best tribology performance. Scratches and generated during the installation process can lead to bursting of the material and the generation of noise (squeaking), chipping, and impingement [36]. MoM constructs reported significant increase in metal ion concentration. For example, normal blood concentrations of cobalt and chromium are less than $0.25 \mu \mathrm{g} / \mathrm{L}(0.005 \mu \mathrm{M})$. The use of large diameter MoM THA results in elevated cobalt and chromium ion levelss in the blood $(4.6 \mu \mathrm{M}$ and $2.3 \mu \mathrm{M})$ and in the local hip synovial fluid $(30 \mu \mathrm{M}$ and $25 \mu \mathrm{M})$ [37]. The lack of a national joint registry in the United States, has limited the ability of researchers to monitor adverse effects of elevated metal ion levels in large populations and causes of THA failure. The British National Joint Registry has reported four main reasons for revision surgery of Total Hip Arthroplasty. These reasons include 44\% implant loosening, 15\% for adverse local tissue reactions, $14 \%$ for osteolysis, and $13 \%$ for wear [38]. Reports from the Australian Joint Registry have found that MoM constructs have a much higher revision rate than MoP [39]. Subsequent clinical data in both Europe and United States provided additional support. Within the United States, the use of MoM for primary total hip arthroplasty (pTHA) decreased from $31 \%$ in 2007 to $1 \%$ in 2012. MoM constructs are virtually nonexistent do to the concerns of local and systemic problems of metal ions 
[40]. Within the past 4 years, reports have identified modularity in femoral components as an additional source of debris and ALTR. Esposito et al., conducted a systematic review that identified four failure mechanism involving taper junctions in THA devices. These include tribocorrosion-associated ALTR, dissociation of taper junction, stem fracture, mismatch of femoral head to a stem with a different trunnion size. Furthermore they emphasized that regardless of material coupling, the principal solid tribocorrosion product is chromium-orthophosphate hydrate [41]; however, retrieval studies dating back to the early 1990s reported clinical concerns at taper interfaces. Collier et al., found that $56.6 \%$ of the mixed alloy implants $(n=30)$ presented corrosion after retrieval (40 months of implantation), while single alloy components presented no evidence of corrosion ( $\mathrm{n}=49$ for Co head on Co Stem and $\mathrm{n}=9$ for Ti head on Ti stem) [42]. In a 2002 multicenter retrieval study of 231 modular implants, mixed head-stem pairs had higher amount of corrosion relative to single alloy components ( $42 \%$ vs $28 \%$ ) [43]. In light of these concerns modular implants have been praised for their intraoperative installation flexibility, fine tuning of patient anatomical features, and restoration of muscle tension [21]. Providing operative flexibility was further pushed in the 1990s that some implants had two modular features connecting the stem to the head with a middle component known as the neck. This second modular interface allows the surgeon to accommodate clinical cases where standard hip design with a fixed neck geometry and head may not give optimum stability and soft tissue balance [21]. Gill et al., suggests that corrosion is higher at the neck-stem taper than at the head-neck taper [44]. In a recent finding of two cohort study, 284 patients with nonmodular neck and 595 patients with a modular neck, found that the use of dual modular stems did not improve hip scores nor reduce the 
likelihood of complications when compared to the monolithic group. The mean follow up time of the study was 2.4 years [45]. The 2011 Australian joint registry reported that overall revision rate for modular femoral stems are double that of fixed neck THA, 8.9\% and $4.2 \%$, respectively [39]. The general consensus, amongst joint replacement surgeons, is that dual-modular tapers should not be used because supposed benefits do not overcome the addition wear and corrosion at a second interface. A matched cohort design of 100 head-stem monolithic pairs (50 ceramic heads and $50 \mathrm{CoCr}$ heads) found that fretting and corrosion was lower ceramic cohort $(\mathrm{p}=0.03)$ [46]. This study further suggests that using a ceramic head may reduce the corrosion at the head/stem taper. Revision surgery can be more challenging. Surgeons have faced difficult situations such as how to revise a THA device without removed a well-fixed stem with a corroded or damaged trunnion. The general consensus amongst total hip replacement surgeons is to it is best to remove the femoral head and remove surrounding necrotic tissue, while leaving a well-fixed yet corroded taper. The alternative is to remove the femoral stem entire and risk shattering the femur. Additionally if possible, re-clean the trunnion and add a ceramic head with a titanium sleeve. Furthermore, the polyethylene liner should be replaced [47]. Nevertheless, in 2013, the orthopedic network news reports that there was approximately 470,000 primary THA surgeries performed in the United States. The hip implant market is projected to grew year over year by over $2 \%$. 


\section{Chapter 3: Cobalt Chromium Molybdenum Alloy}

\subsection{A Brief History of CoCrMo Alloys}

In 1907, Elwood Haynes patented the development of cobalt-chromium (CoCr) alloys known as Stellite. He also discovered that adding tungsten (W) and molybdenum (Mo) could improve the mechanical properties of $\mathrm{CoCr}$ alloys [48]. This alloy is obtained first by reducing cobalt and chromium ores through extraction metallurgy. The pure metals are combined under vacuum by electric arc or induction melting. The chemical reactivity towards oxygen requires the process at high temperature and pressures to occur under a vacuum with inert atmosphere. Combining the two elements, to form $\mathrm{CoCr}$ alloys, became the first example of a superalloy. Superalloy is the classification for highperformance alloys with excellent combination of mechanical strength, corrosion/oxidation resistance, and resistance to thermal creep deformation. The initial development of these CoCr alloys, for engines in the automotive and aviation industry, was hindered by the development of nickel-based superalloys. Nevertheless, CoCr alloys found a niche in biomedical applications [49]. Cobalt-chromium-molybdenum (CoCrMo) alloys were first introduced into orthopedics and dentistry back in the 1930s under the trade name Vitallium. CoCrMo alloys have been utilized in artificial heart valves, dental prosthesis, orthopedic fixation plats, artificial joint components, and vascular stents [50]. Investment cast CoCrMo alloys similar to the 1976 ASTM specification F-75 include H.S. 21, Vinertia, Vitallium, and Protasul [51]; however, these alloys were far from perfect, as was evident in the early 1970s when clinical case studies reported weight bearing failure of these prostheses [52]. Nevertheless, CoCrMo alloy has been one of the 
load-bearing materials of choice for joint replacement surgery as indicated by its clinical usage for over the last forty years as hip and knee replacement devices [53].

\subsection{ASTM Requirements of CoCrMo Alloys}

The American Society for Testing and Materials (ASTM) has standardized the chemical, mechanical, and metallurgical requirements of CoCrMo related materials for surgical implants as part of their ASTM Section 13 - Medical and Surgical Materials and Devices. The chemical composition of the CoCrMo alloy has a balanced weight percentage cobalt, 28-weight percentage chromium, and 6-weight percentage molybdenum (Co-28Cr-6Mo). ASTM F75 is the standard for casting production. Casting is a manufacturing technique that can be used to form products with complex shapes in a cost-efficient manner. The basis of casting begins with the use an expendable material to form a mold/cavity around a ceramic like slurry. When the slurry hardens, the mold (generally made from a plastic or wax) is removed and molten (liquid) metal is poured into the ceramic mold [54]. ASTM F1573 is the standard for wrought product. Wrought production implies that a metal is worked (hammered/pressed) into a desired shaped [55]. Additionally, F799 is the standard for forge production. The forged alloy has a chemical composition following the ASTM F1573 standard. Forging is a thermomechanical processing to improve mechanical properties [56]. The chemical composition of ASTM F75 and F1537 is reproduced in table 2.1. The mechanical properties are reported in table 2.2 . 


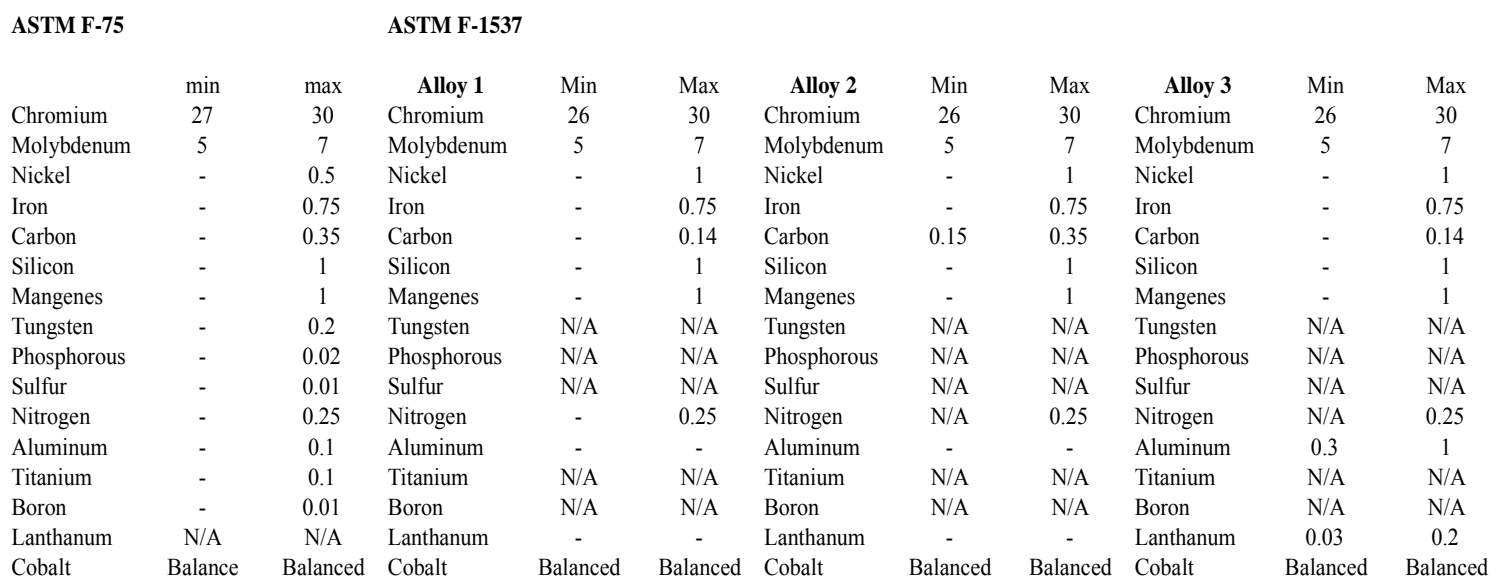

Table 2.1: Chemical Composition of ASTM F75 and ASTM F-1537

$\begin{array}{llccccc}\text { ASTM Standard Processing Method } & \text { Tensile Strength MPa } & \text { Yield Strength MPa } & \text { Elongation \% } & \text { Reductio of Area \% Hardness HV } \\ \text { F-75 } & \text { As-Cast } & 655 & 450 & 8 & 8 & \text { N/A } \\ \text { F-1537 } & \text { Wrought/Annealed } & 897 & 517 & 20 & 20 & 260 \\ & \text { Wrought/Hot Worked } & 1000 & 700 & 12 & 12 & 280 \\ & \text { Wrought/Warm Worked } & 1192 & 827 & 12 & 12 & 340 \\ \text { F-799 } & \text { Wrought/Forged } & 1172 & 827 & 12 & 12 & 340\end{array}$

Table 2.2: Mechanical Properties as reported in ASTM F75, F1537, and F799

ASTM F75 accepts various thermal treatments such as hot isostatic pressing (HIP), solution annealing, and sintering during the manufacturing of this casting alloy; however, how these treatments influence the mechanical properties of this standard is not reported in the astm standard. Furthermore ASTM F75 is similar to ISO 5832-4 [54]. ASTM F1537 has three sub-alloy chemical compositions. Alloy 1 is classified as a low-carbon alloy system. Alloy 2 is classified as a high-carbon alloy system. Alloy 3 is classified as a dispersion-strengthened alloy containing fine aluminum-lanthanum oxides. It should be further noted that the minimum mechanical properties of wrought production ASTM F1537 is superior to the minimum mechanical properties of cast ASTM F75 [55]. Forging is a thermomechanical method to develop finer microstructure grain-size, improve the alloy's homogeneity, and impart strain-induced hardening. Thus the mechanical 
properties of forging ASTM F799 are drastically improved compared to casting ASTM F75 [56].

\subsection{CoCrMo Phases}

Pure cobalt undergoes allotropic transformation from the metastable face center cubic (FCC) crystal structure to the thermodynamically stable hexagon closed packed (HCP) crystal structure at around $417^{\circ} \mathrm{C}$, as illustrated in figure 3.1 . The FCC phase, also known as $\gamma$-Co is stable above $417^{\circ} \mathrm{C}$, while the HCP phase, also known as $\varepsilon$-Co, is stable below $417^{\circ} \mathrm{C}$.

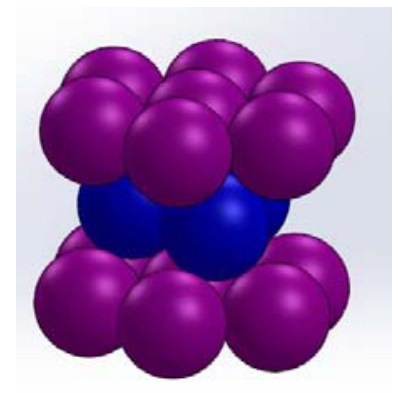

Hexagonal Closed Packed (HCP)

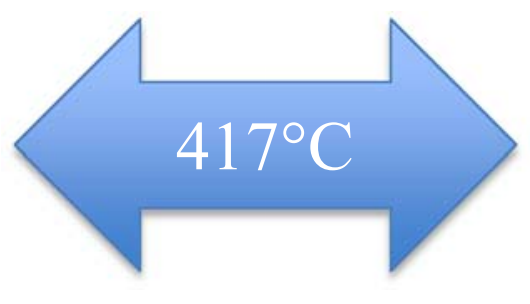

Face Center Cubic (FCC)

Figure 3.1: Crystal Structure Representation of HCP and FCC

Pure Co enters the liquidus phase at $1495^{\circ} \mathrm{C}$. During normal cooling rates, after processing, the phase transformation is quite sluggish has been suggested to occur by martensite mechanism [57]. For the Co-27Cr-5Mo-0.05C alloy (similar to the ASTM alloys), the transformation temperature shits to $970^{\circ} \mathrm{C}$. Figures 3.2 and 3.3 are the binary phase diagrams for $\mathrm{Co}-\mathrm{Cr}$ and $\mathrm{Co}-\mathrm{Mo}$, respectively. Both diagrams highlight the relationship between temperature and concentration as a function of phase stability. For the purposes of this study, it is only necessary to analyze these binary phase diagrams of 
Co-Cr around (26-30 wt\% of $\mathrm{Cr}$ ) and Co-Mo around 5-7\% wt of Mo). Both diagrams show that chromium and molybdenum are $\varepsilon$-Co stabilizers. Increasing concentration of either element will raise the transition temperature from HCP to FCC.

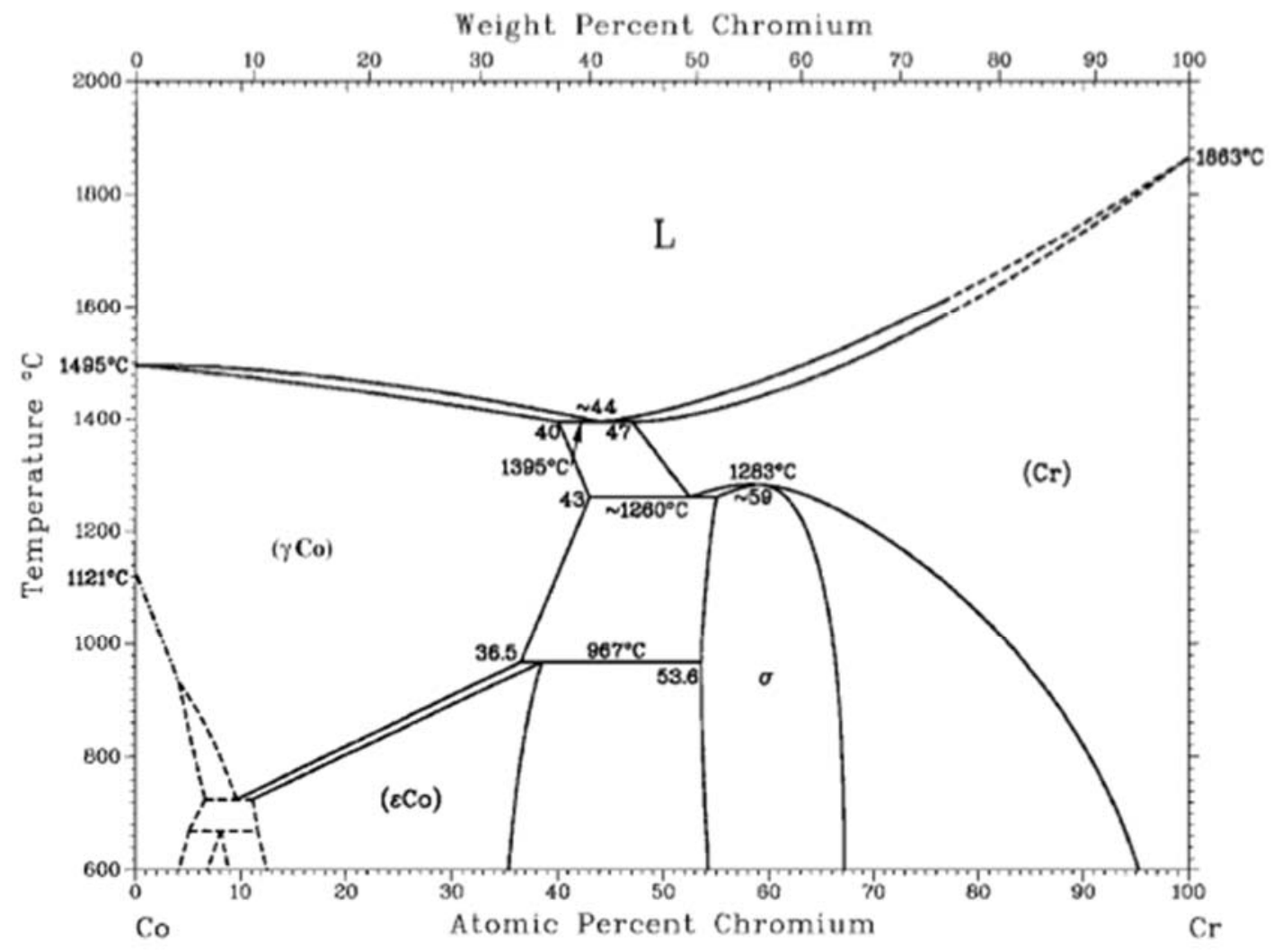

Figure 3.2: Co-Cr Binary Phase Diagram [58] 


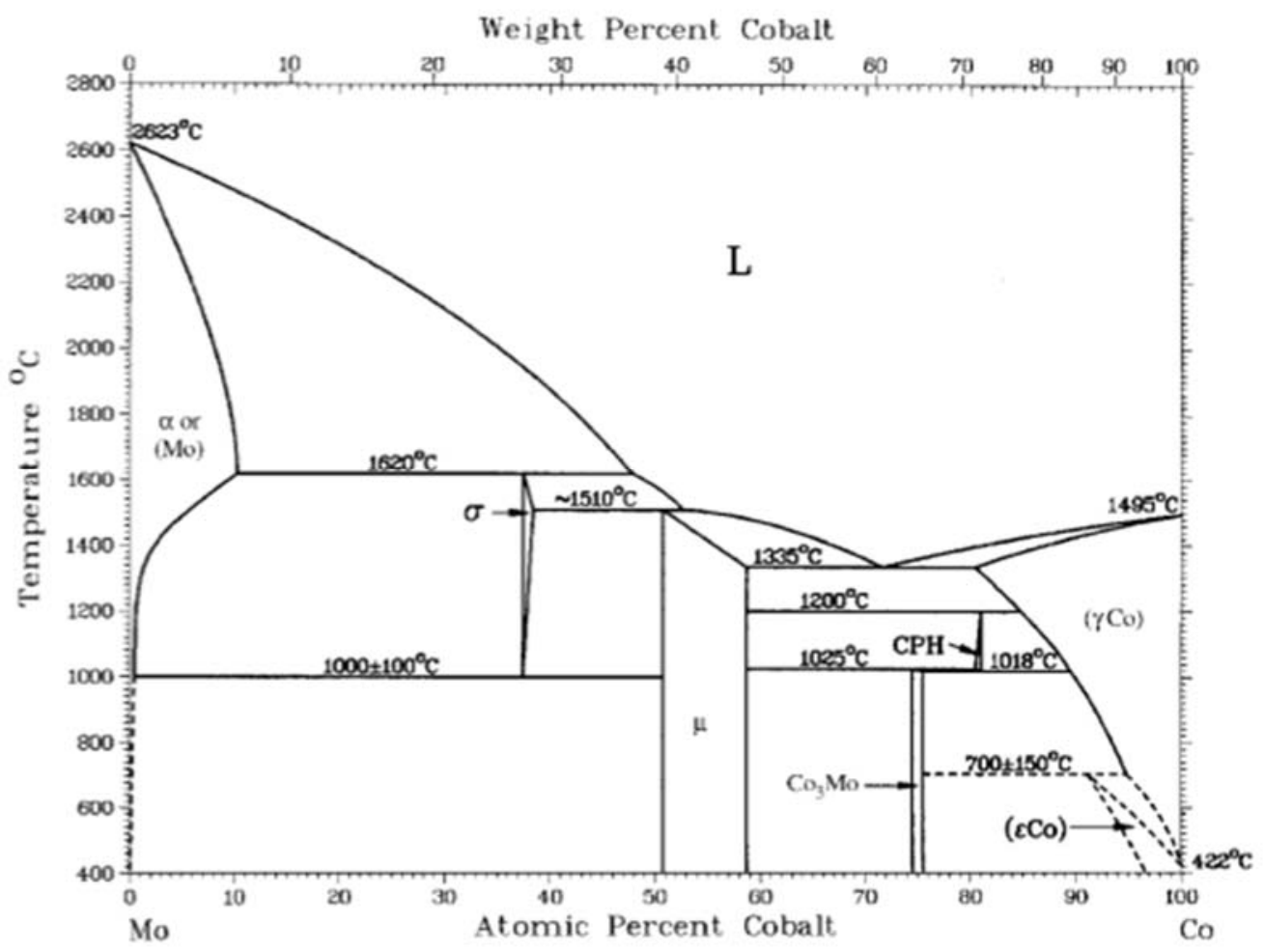

Figure 3.3: Co-Mo Binary Phase Diagram [58]

Chromium is one of the most important alloying elements because it provides the alloy with excellent corrosion resistance by forming the $\mathrm{Cr}_{2} \mathrm{O}_{3}$ passive oxide film on the surface. Molybdenum is added because in solid solution, it can refine the crystal grains and improve the tensile strength [59]. Carbon is an equally important element in CoCrMo systems. Carbon content is an important distinguishing point because low carbon versions of this alloy have a soluble carbon content of less than $0.15 \mathrm{wt} \%$ carbon; however, high carbon alloys are allowed to have up to $0.35 \mathrm{wt} \%$ carbon. From table 2.1, ASTM F75 has a maximum carbon content of $0.35 \mathrm{wt} \%$. ASTM F1535 separates low carbon (alloy 1) from high carbon (alloy 2) by carbon weight percentage. Carbon is solution forms metalcarbides (MC). Where $\mathrm{M}$ tends to be chromium and molybdenum. The increased in carbon content results in a significantly higher volume fraction of carbides. Carbides in 
CoCrMo alloy have the formula of $\mathrm{M}_{23} \mathrm{C}_{7}$. Carbide precipitation has been one of the main strengthening mechanisms for the ASTM F75 alloy [60]. In general, carbides increase strength, hardness, while reducing ductility of CoCrMo alloys. High carbon alloys tend to display excellent wear resistant as attributed to their higher hardness. Furthermore, it has also been reported that increasing the nitrogen $(0.04$ to $0.14 \mathrm{wt} \%)$ content in solution for CoCrMo alloys increases the tensile and fatigue strength, slightly increases the hardness, while decreasing ductility [61].

\subsection{Microstructure}

Processing techniques used to fabricate a final product influences the mechanical properties of metals. Thermal and mechanical strains can influence the microstructure of CoCrMo materials and ultimately the material properties. The microstructure can provide an insight into the processing history of the metallic sample of interest. One important relationship is between microstructure and mechanical properties. The microstructure of an alloy is a detailed view of grains and (orderly array of crystal lattices in space) and grain boundaries (higher energy sites with great atomic distortions). Grain boundaries act as impeding points to further dislocation propagation. The hall-petch equation is an inverse approximation between grain size and yield strength. Smaller grains result in a higher yield strength for the material. Grain size and second phase precipitates can affect strength, ductility, and wear resistance. Metallurgical methods can be used to adjust these properties [62]. Another mechanism to improve mechanical properties of homogenous alloys (single phase solid solutions) is through solid solution hardening. Heterogeneous alloys (multiphase alloys) mechanical strength is determined by the size and distribution of the different phases. The fabrication of implants casting of Co-Cr based alloys is not a 
preferred technique, as solidification during casting results in large dendritic grains [63]. The large dendritic grains decrease the yield strength of the alloy. Additionally casting defects such as inclusions and micropores cannot be avoided and may act as stress risers. The CoCrMo as-cast F75 alloy has been in alloy used in orthopedic implants since the 1950s. Hot-isostatic pressing (HIP) and homogenization heat treatments have been routinely used to improve the mechanical properties by reducing the porosity in castings. Hot isostatic pressing can sinter fine powders that are then forged to a final shape [64]. Typical microstructures after forging is 8 microns - significantly smaller than the as cast alloy [65]. Figures 3.4 and 3.5 shows typical as-cast (F75) and wrought (F1537) Co28Cr-6Mo microstructures, respectively. As-cast microstructures tend to display a lamellar or dendritic microstructure, whereas wrought alloys to show an equiaxial structure.
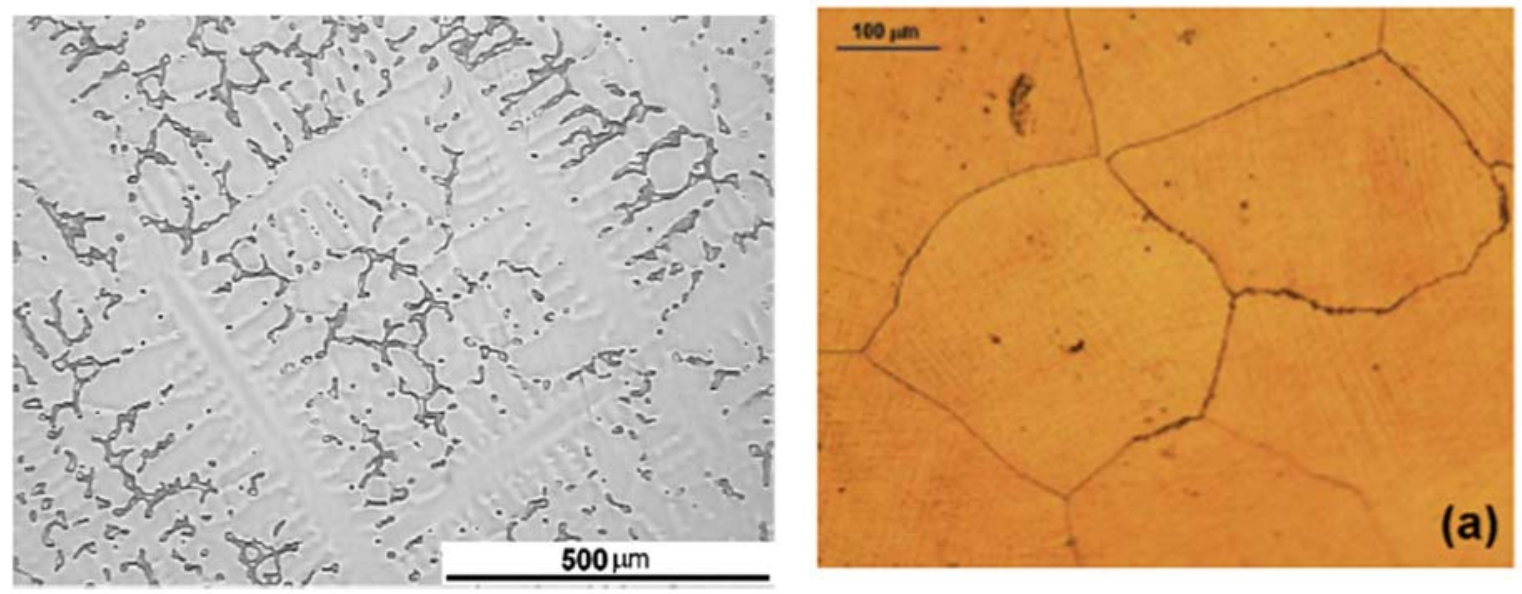

Figure 3.4: Typical As-Cast Microstructure (left) [66];

Figure 3.5: Typical Wrought Microstructure (right) [67] 


\subsection{Processing}

The microstructure of F-75 is composed of a cobalt-based faced-center cubic (FCC) matrix with interdendritic and grain boundary precipitates [60]. It has been established that the precipitates can be a mixture of metal carbides $\left(\mathrm{M}_{23} \mathrm{C}_{6}\right)$, sigma $(\sigma)$ intermetallic phase, and FCC phase [51]. Carbide precipitation is generated to strengthen the as-cast F75 alloy; however, this is also known to lower ductility. Lamellar carbide precipitation at the grain boundaries, also "perlite type" precipitates, can result from slow cooling rates during the solidification process or posterior heat treatments [68]. Short (1-4hours) thermal treatments after carbide formation at around $1200^{\circ} \mathrm{C}(1473 \mathrm{~K})$ can help partially dissolve the carbides to improve ductility, yield strength, and ultimate tensile strength. Extend heat treatment period's lead to excess ductility with loss of other mechanical properties [69]. The chemical composition of the alloy along with the temperature and duration of the heat treatment has created discrepancies and variances as to reported improvements of mechanical properties. Interdendritic precipitates solidify around $1223^{\circ} \mathrm{C},(1496 \mathrm{~K})$ and that $\mathrm{M}_{23} \mathrm{C}_{6}$ precipitates can dissolve directly into the matrix as shown in figure $3.6[60]$.

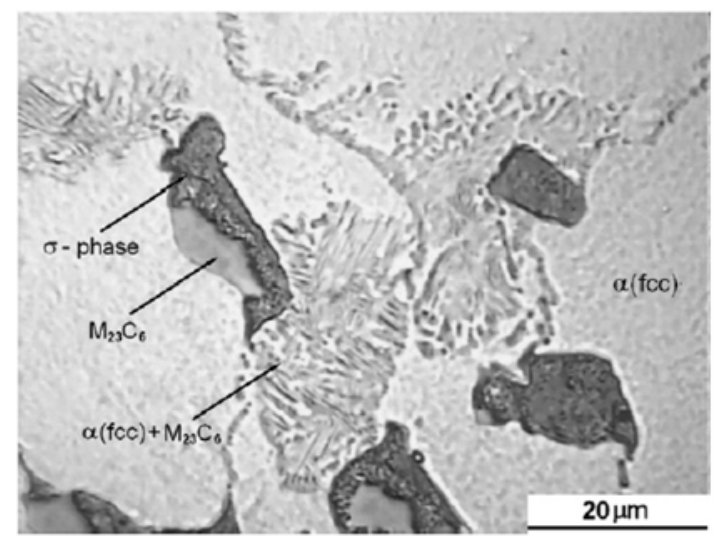

Figure 3.6: Carbides and Phases in CoCrMo Microstructure 
The primary deformation of the as-cast F-75 alloy occurs through the presence of wide stacking faults and twins. These features contribute to the flow stress in the alloy and subsequently work hardening mechanism. The formation of stacking faults and twins are the structural precursors to the $\mathrm{FCC} \rightarrow \mathrm{HCP}$ transformation at room temperature [70]. It has been suggested that preheating an as-cast material at different temperatures can result in different carbide morphologies. Alloy preheating (4 hours) at $815^{\circ} \mathrm{C}$ shows eutectic carbides of blocky and globular morphology. Preheating at $950^{\circ} \mathrm{C}$ results in carbide precipitation at interdendritic boundaries. At $1100^{\circ} \mathrm{C}$ preheat, carbide morphology can be characterized as carbide spheroidization. Furthermore, solid solution treated at $1225^{\circ} \mathrm{C}(4$ hours) can create large equiaxial grain structures with fine precipitates dispersed inside the grains and at the grain boundaries. It was found that preheat at $815^{\circ} \mathrm{C}$ followed by solid solution treatment at $1225^{\circ} \mathrm{C}$ lead to maximum ductility and alloy strength [71]. In pure cobalt, FCC is the metastable phase and transition to HCP upon cooling to room temperature. This takes place at around $417^{\circ} \mathrm{C}$ by a by the sluggish martensitic process [57]. For Co-Cr-Mo-C alloy, the FCC metastable phase is retained at room temperature. At around $1220^{\circ} \mathrm{C} \sigma$ phase precipitates and it transforms to $\mathrm{M}_{23} \mathrm{C}_{6}$ carbides. 


\section{Chapter 4: Electrochemical and Corrosion Behavior of CoCrMo Alloys}

\subsection{Total Hip Arthroplasty Corrosion}

Adverse effects associated with THA range from mild pain to complete failure of implant. Research has found that one of the main underlying cause of pain, implant loosing, and/or implant failure results from localized corrosion. Various groups have offered excellent theories to the causes of corrosion and have utilized various resources to determine which factors are prone to accelerate corrosion. Many investigators (surgeons, research physicians) view corrosion from a clinical perspective. Some of the clinical concerns of a corroding implant include: elevated metal ion concentration in synovial fluid and blood, pseudotumor and necrotic black tissue around implants, and extraordinary patient discomfort as a result of implant loosening or fracture [72]. Although factors such as manufacturing conditions and tolerances and operator skill during implantation are additional variables to the potential causes of implant failure as emphasized by retrieval studies, implant corrosion is a phenomenon that inherently affects the outcome of all patients with implants. Furthermore, no metal implant can avoid corroding. Mitigating an implants susceptibility to corrosion can improve longterm patient outcomes. Thereby, it is important to review corrosion theory.

\subsection{Corrosion Thermodynamics}

Most metals and alloys used in industry application are naturally found as ores - a metal oxide state. For pure metals, such as cobalt, chromium, and molybdenum, the metal oxide state is the most thermodynamically stable condition, meaning, the metal oxide phase is the lowest energy level. Metals are purified in a process known as extraction metallurgy under high temperature and pressure within a reducing atmosphere. Thus pure metals are 
less thermodynamically stable than metal-oxides. Metals exist in a metastable state because they are at higher energy levels than metal oxides. Kinetic barriers such as passive oxide layer prevent pure metals from instantaneous oxidation under standard temperature and pressure conditions. Many pure metals are alloyed to produce enhanced material properties [73]. In engineering applications, corrosion is viewed as an unwarranted electrochemical degradation of a material with the surrounding environment. Most metals, except the noble metals, are susceptible to corrosion. Corrosion is unfavorable because the material loss structural and functional integrity; however, corrosion should also be viewed as the natural and reversible process of extraction metallurgy whereby metals return to their lower energy oxide state. Corrosion is a surface phenomenon where by the metal and the surrounding environment undergo a series of oxidation-reduction (redox) reactions, involving the transfer of electrons in an electrolytic solution [74]. Thus, corrosion involves the simultaneous mass and charge transfer between metal/solution interfaces. A simple half-cell redox reaction is shown in equations 1-3.

Metal Oxidation (anodic reaction)

Hydrogen Ion Reduction (cathodic reaction)

Net Reaction

$$
\begin{aligned}
& \mathrm{M} \rightarrow \mathrm{M}^{2+}+2 \mathrm{e}^{-} \\
& 2 \mathrm{H}^{+}+2 \mathrm{e}^{-} \rightarrow \mathrm{H}_{2} \\
& \mathrm{M}+2 \mathrm{H}^{+} \rightarrow \mathrm{H}_{2}+\mathrm{M}^{2+}
\end{aligned}
$$

An oxidation (anodic) reaction is the generation of electrons. In all metallic corrosion processes, the rate of the anodic reaction must equal the rate of the cathodic reaction. 
Equation 2 above is also known as hydrogen evolution. A few common cathodic reactions, equations 3-4, include:

$$
\begin{array}{ll}
\text { Oxygen reduction }(\mathrm{pH}<7) & \mathrm{O}_{2}+4 \mathrm{H}^{+}+2 e^{-} \rightarrow 2 \mathrm{H}_{2} \mathrm{O} \\
\text { Water reduction }(\mathrm{pH} \geq 7) & \mathrm{O}_{2}+2 \mathrm{H}_{2} \mathrm{O}+2 e^{-} \rightarrow 4 \mathrm{OH}^{-}
\end{array}
$$

Equations 1-4 are were reproduced from Fontana et. al [75]. Corrosion thermodynamics is the driving force that causes metals to degrade because of a difference in energy level between the metal and metal oxide state. Thermodynamics can be used to determine whether or not a corrosion process will occur. Thermodynamics is the study of energy. All systems seek to exist in the lowest energy state (ground state). Lowering one's energy state can be accomplished by releasing energy. The change in Gibbs Free Energy $(\Delta \mathrm{G})$ will ultimately determine if a reaction will spontaneously occur. $\Delta \mathrm{G}$ is defined by equation 5.

$\Delta G=\Delta H-T \Delta S(5)$

$\Delta G=$ Change in Gibbs Free Energy; negative values indicate spontaneous reactions

$\Delta H=$ Change in enthalpy

$\mathrm{T}=$ Temperature and is assumed to be constant.

$\Delta S=$ Change in entropy

The derivation of equation 5 can be obtained here [76]. 
Thus a spontaneous reaction is thermodynamically favorable because energy is released from the system. The free energy change for an electrochemical process [77] is defined as

$\Delta G=-n F E(6)$

$\mathrm{n}=$ number of electrons transferred

$\mathrm{F}=$ Faraday's constant

$\mathrm{E}=$ half cell potential

For most metals, corrosion is a spontaneous process. This concept can be better understood through a simple analysis of the electromotive force series (emf) of reduction reactions for metals. The emf values for the main metals in the alloy CoCrMo are listed below in order from less active species [78].
$\mathrm{H}_{2} \rightarrow 2 \mathrm{H}^{+}+2 \mathrm{e}^{-}$
$\mathrm{E}=0.000 \mathrm{~V}$
(standard hydrogen electrode; SHE)
$\mathrm{Mo} \rightarrow \mathrm{Mo}^{3}++3 \mathrm{e}^{-}$
$\mathrm{E}=0.200 \mathrm{~V}$
$\mathrm{Co} \rightarrow \mathrm{Co}^{2+}+2 \mathrm{e}^{-}$
$\mathrm{E}=0.280 \mathrm{~V}$
$\mathrm{Cr} \rightarrow \mathrm{Cr}^{3+}+3 \mathrm{e}^{-}$
$\mathrm{E}=0.744 \mathrm{~V}$

Because the potentials of the three-oxidation reaction are positive, from equation 6 , this results in negative free energy change - oxidation of the three pure elements (equation 810) is spontaneous. The utilization of the EMF series is quite limited since several key assumption are made such as unit activity, reactivity varies in different media, and that the emf series can only be applied to pure metals and never alloys. Many reactions are 
not at 1 molar concentration (unity). Therefore, a more useful equation, equation 10, in corrosion thermodynamics is the Nernst Equation -equation 10 [83]:

$E=E^{\circ}-\frac{2.303 R T}{n F} \log (K)$

Where $\mathrm{E}$ is the potential for a reaction not a unity. $\mathrm{E}^{\mathrm{O}}$ is the potential of 1 molar concentration. $\mathrm{R}$ is the universal gas constant. $\mathrm{T}$ is temperature in kelvin. $\mathrm{K}$ is the equilibrium constant for a reaction. The most useful application of the Nernst equation are Pourbaix diagrams. Marcel Pourbaix plot potential vs $\mathrm{pH}$ to determine which species is most thermodynamically stable at a particular potential and $\mathrm{pH}$. Pourbaix diagrams can be used to predict regions of immunity (pure metal), passivity (metal oxide), or corrosion (metal ion). These diagrams are built from the Nernst Equation. Pourbaix diagrams do not provide actual corrosion rates, but they can be used to provide thermodynamic trends to be verified with experimental results. The most important aspect to grasp with corrosion thermodynamics is its limitation. Corrosion of a metal is a non-equilibrium reaction. Experiments are required to determine corrosion rates.

\subsection{Corrosion Kinetics}

Corrosion thermodynamics is excellent at determining if a corrosion process will occur; however, from an engineering application standpoint, corrosion kinetics - the rate of corrosion - is perhaps more important. A corrosion processes may be thermodynamically favorable but a large energy barrier can prevent the reaction from proceeding. Furthermore corrosion reactions are not at equilibrium, a required parameter in thermodynamics. Short-circuiting two half-cell electrodes is one method to measure corrosion. The potential of the individual electrodes will no longer be at their equilibrium potentials. The deviation from the equilibrium potential is known as polarization. Since 
oxidation and reduction reaction are equal the electron exchange rate can be measured in current density. Faraday's law defines the relationship between reaction rate and current density as defined by equation 11 :

$r_{\text {red }}=r_{o x i}=\frac{i_{o}}{n F}$

red $=$ the equilibrium reduction rate

$r_{\text {oxi }}=$ the equilibrium oxidation rate

$i_{0}=$ the exchange current density

Polarization of the electrode can be determined by two mechanisms activation polarization and concentration polarization. Activation polarization occurs when electron transfer is the slowest step as defined by equation 12 and is also known as Tafel's equation.

$\eta_{a}= \pm \beta \log \frac{i}{i_{o}}$

$\eta_{a=}$ activation polarization overvoltage

$\beta=$ Tafel's constant

$i=$ rate of oxidation or reduction in terms of current density 
Concentration polarization occurs when diffusion of some ionic species (hydrogen ions) is the limiting rate. This is know as limiting diffusion current density

$i_{L}=\frac{D n F C_{B}}{x}$

$i_{\mathrm{L}}=$ limiting diffusion current density

$\mathrm{D}=$ diffusion coefficient of the reacting ions

$\mathrm{C}_{\mathrm{B}}=$ the concentration of the reacting ions in bulk solution

$\mathrm{x}=$ thickness of the diffusion layer

Concentration polarization is defined in equation 14:

$\eta_{c}=2.3 \frac{R T}{n F} \log \left(1-\frac{i}{i_{L}}\right)$

$\eta_{\mathrm{c}}=$ activation polarization overvoltage

The kinetics of every corrosion reaction can be determined by known $\beta$, io, and iL.

Total Polarization $\left(\eta_{\mathrm{T}}\right)$ is the summation of concentration and activation polarization and defined by equation 15 :

$\eta_{T}=\eta_{a}+\eta_{c}$ 
Equations 11-15 were referenced from Corrosion Engineering [79].

Mixed potential theory states that the reduction reaction must equal the oxidation reaction for the corrosion of an electrically isolated metal sample. This equilibrium will provide the corrosion potential $\mathrm{E}_{\mathrm{corr}}$ and corrosion current density $\mathrm{i}_{\text {corr. }}$ If $\beta$ values and exchange current density of the system is known then corrosion rates can be determined. At this point a few key terms are necessary to be defined. Anode refers to the electrode at which oxidation occurs. Cathode refers to the electrode at which reduction occurs. Furthermore anodic reaction is equal to oxidation reaction and cathodic reaction is the same as reduction reactions.

Faraday's law (equation 16) states that the mass (W) of metal corroded,

$W=\frac{I t A}{n F}$

$\mathrm{I}=$ current in amperes

$\mathrm{t}=$ time in seconds

$\mathrm{A}=$ the atomic weight of the metal

$\mathrm{n}=$ the number of equivalents transferred per mole of metal

Corrosion rates are expressed in mils per year by in equation 17 . 
$m p y=\frac{534 W}{D A T}$

$\mathrm{W}=$ weight loss in $\mathrm{mg}$

$\mathrm{D}=$ density $\mathrm{g} / \mathrm{cm}^{3}$

$\mathrm{A}=\operatorname{area~in~}^{2}$

$\mathrm{T}=$ exposure time in hours

A corrosion rate of $1 \mu \mathrm{A} / \mathrm{cm}^{2}$ approximates $1 \mathrm{mpy}$ for most metals. Additionally corrosion current densities greater than $100 \mu \mathrm{A} / \mathrm{cm}^{2}$ are considered unacceptable. Equations 16 and 17 are referenced from [80].

\subsection{Forms of Corrosion}

Fontana et al., highlighted eight unique corrosion mechanisms: 1) uniform or general attack, 2) galvanic corrosion, 3) crevice corrosion, 4) pitting, 5) intergranular corrosion, 6) selective leaching, 7) erosion corrosion, and 8) stress crack corrosion [81]. Although corrosion at its core is the oxidation of metal, the process by which it occurs, varies. Galvanic corrosion, crevice corrosion, pitting corrosion, and intergranular corrosion have been reported in clinical journals as possible failure mechanisms. At this moment it is worth differentiating them.

Galvanic corrosion occurs when two dissimilar metals are immersed in a conductive solution. This is because there is a potential difference between the two metals. If the metals are placed in contact, the potential difference causes electrons to flow between the metals. The more active metal becomes anodic and the more noble metal becomes 
cathodic. Potentials between metals are determined by taking the absolute difference between their standard emf potentials; however, the galvanic series is a better indicator than emf series to determine corrosion susceptibility. Titanium and its alloys are significantly nobler than cobalt-chromium based alloys. Potentials generated by a galvanic cell of dissimilar metals may change with time. In galvanic corrosion, polarization of the reduction reaction (cathodic polarization) usually predominates. Environment, distance from dissimilar metal junction, and area effect (current density is greater for a small electrode than for a larger one) all play a role in galvanic corrosion process. Galvanic corrosion can be one corrosion mechanism that occurs at taper junctions of modular implants, especially when CoCrMo alloy femoral heads are mated to titanium alloy femoral stems [81].

Crevice corrosion is an intensive localized corrosive process that occurs within crevices and other shielded areas on metal surfaces exposed to oxidizing environments. Additionally, crevice corrosion occurs in many mediums, although it is usually most intense in ones containing chloride ions. It is associated with small volumes of stagnant solution caused by holes and cracks. To function as a corrosion site, a crevice must be wide enough to permit liquid entry but narrow enough to maintain a stagnant zone. As metal is oxidized, and oxygen from solution is reduced, the concentration of oxygen becomes depleted. A large depletion of oxygen, without replenishment, cause a higher concentration of positively charged metal ions. To maintain charge neutrality, chloride ions tend to migrate to the crevice. The fluid within crevices exposed to neutral dilute sodium chloride $(\mathrm{NaCl})$ solutions has been observed to contain 3 to 10 times as much 
chloride as the bulk solution and to posses a $\mathrm{pH}$ of 2 to 3 . As the corrosion within the crevice increases, the rate of oxygen reduction on adjacent surfaces also increases. Crevice corrosion attacks localized sites, while the remaining bulk structure can be unaffected. Crevice corrosion can be delayed for some time; however, once started the mechanism accelerates quickly [81].

Pitting corrosion is a form of extremely localized attack that results in holes in the metal. Pitting corrosion is similar to crevice corrosion. Pits are isolated or close together and look like a rough surface. A pit may be described as a cavity or hole with the surface diameter about the same as or less than its depth. Pitting is most destructive because it causes equipment to fail with only a small percent weight loss of the entire structure. Pitting is difficult to predict by laboratory tests and tend to grown in the direction of gravitational force. Corrosion pit is a unique type of anodic reaction because it is selfstimulating and self-propagating. Most pitting failures are caused by chloride and chlorine-containing ions [81].

Grain boundaries are of a higher energy state than grains. The difference may not be significant under uniform attack. Under certain conditions, grain interfaces can be of significantly higher energy state, compared to grains, and thus very reactive. This form of localized corrosion based on heterogeneity within the solid solution of an alloy is known as intergranular corrosion. This form of corrosion occurs along the grain boundaries. Intergranular corrosion can be caused by impurities at the grain boundaries, enrichment of one of the alloying elements, or depletion of one of these elements in the grain- 
boundary areas. For example, depletion of chromium in the grain-boundary regions results in intergranular corrosion of stainless steels and cobalt-chromium alloys. Carbide precipitation can deplete passivation elements from corrosion. Although carbides themselves are quite corrosion resistant, the regions around carbides can be depleted of chromium. These areas may not have enough chromium to resist oxidation [81].

\subsection{Corrosion of CoCrMo Implants}

CoCrMo alloys forms a protective oxide $\left(\mathrm{Cr}_{2} \mathrm{O}_{3}\right)$ layer. The protective nature of this film is determined by the film's composition, structure, thickness, and the amount of defects present. Additionally, environmental factors ( $\mathrm{pH}$, chloride ions, exposure duration, etc.) can alter the protectiveness of this film. The corrosion behavior of such alloys is influenced by several factors that include material characteristics (chemical composition, microstructure, surface features), external environment $(\mathrm{pH}$, temperature, oxygen content), and manufacturing conditions (cold working and thermal treatments) [82]. The passive film of CoCrMo is the kinetic limiting barrier to corrosion in aqueous solution. It acts as a physical barrier to both cation and anion transport to the metal surface, and a barrier to electron dissolution. The human body is a highly corrosive environment for metals and this oxide film is the barrier between a well functioning hip replacement and revision surgery. Body fluids (human plasma, synovial fluid of joints) are composed of $0.9 \%$ sodium chloride $(\mathrm{NaCl})$ with additional inorganic salts and various protein molecules (synovial fluid has hyaluronic acid and lubricin, and the plasma has albumin, transferrin, globulin, fibrinogen) [82]. Body fluids are highly corrosive in nature as a result of the high concentration of chlorine ions in solution, which can accelerate pitting corrosion. Such forms of localized corrosion attack inhomogeneity found along the 
material surface (passive oxide scratches) and high-energy regions of the alloy's microstructure such as grain boundaries. Additionally, crevices such as found between components joint prosthesis, and inflammation after prosthesis implantation can result in significantly lower $\mathrm{pH}$ values $(\mathrm{pH}<5)$ that can accelerate crevice corrosion. Furthermore $\mathrm{pH}$ in tissue surrounding aseptic and septic loosening can have a 1000 fold greater concentration of hydrogen ions $\left(\mathrm{H}^{+}\right)$[83]. CoCrMo alloys typically fail by transpassive dissolution. In vitro studies of CoCrMo have shown transpassive dissolution of the alloy leads to activation of the surface due to oxidation of the insoluble $\mathrm{Cr}$ (III) oxide layer to the soluble $\operatorname{Cr}(\mathrm{IV})$ species [84]. Electrochemical simulated experiments have reported metastable pitting corrosion in the form of current transients in the region of passivity. For example, pitting nucleation takes place, however, pitting propagation to form large cavities fails. Relatively fast repassivation prevents deterioration of the system [83]. Previous investigation of the electrochemistry properties of CoCrMo alloys has been tested with a variety of simulated physiological solutions (SPS). These solutions include simple isotonic saline $0.9 \% \mathrm{NaCl}$ [85-87] to phosphate buffer saline [67, 88-91], ringer's solution, and hanks solution [92-95]. Ionic solutions similar to body fluids require the addition of biomolecules such as proteins, phospholipids, and glycoproteins. Studies tend to add serum proteins to the electrolyte. CoCrMo alloys spontaneously form a thin passive layer in an oxygen rich environment. The passivation potential is approximately between $-0.3 \mathrm{~V}$ to $+0.5 \mathrm{~V}$ vs standard calomel electrode (SCE) at more positive potentials, the alloy will begin to experience transpassive oxidation [95]. This passivation behavior is profound in many types of simulated physiological solutions (SPS); however differences have been found. For instance, the presence of phosphate ions tends to lower 
the transpassive range to more negative potentials [87]. The explanation for this behavior is the $\mathrm{Cr}(\mathrm{IV})$ species is formed in the presence of phosphate ions due to the creation of $\mathrm{CrPO}_{4}$ layer. It has been found that phosphate ions adsorb strongly on CoCrMo [96]. The passivation of Co is limited to alkaline solutions, with hardly occurs in vivo since biological $\mathrm{pH}$ is tightly regulated at 7.4 ; thereby, $\mathrm{Cr}_{2} \mathrm{O}_{3}$ tends to be the key alloying element responsible for the corrosion resistance of CoCrMo. Additionally Co and Mo oxides make minor contributions to the oxide film [27, 53]. Acidity of solution has an important influence on the formation of the oxide layer. One group reported that the repassivation rate decreased with increasing $\mathrm{pH}$ as unstable Co oxides hinder the formation of chromium oxide passive film [97]. Angle resolved X-ray photoelectron spectroscopy (XPS) indicates that $\mathrm{Cr}$ and Mo oxides are formed on the outer layer whereas Co oxide is present mainly in the interior oxide part [96]. The passive films have been described in detail by surface techniques such as X-ray photoelectron spectroscopy [99] and auger electron spectroscopy [97]. The absorption of biomolecules onto the passive layer of CoCrMo has been found with mixed opinions. For example, one group has suggested that the presence of proteins can act as an accelerated to corrosion [98], while other have found that this new biofilm (a combination of proteins and surface oxide layer) acts as a lubricating film reducing both the coefficient of friction articulating surface and loss of wear material [99], thus reducing degradation. It is important to be mindful the different variables that can alter the corrosion behavior of CoCrMo alloys. 


\section{Chapter 5: Materials and Methods}

\subsection{Sample Preparation}

CoCrMo metallurgical samples were sectioned from explanted hip prostheses. The stem and the head components of each implant were first separated. A linear precision saw (ISOMET 4000) with a diamond tipped blade was used to make cross sectional cuts to both the femoral stem and femoral head components. Each component was secured to a vice, which is fastened to a rigid component within the cutting chamber of the high-speed saw. Lubricant/coolant was mixed with standard tap water that provided a continuous removal of excess heat that may generate during the cutting cycles. Additionally, this may prevent any microstructural changes caused by thermal strains. Furthermore, the stream of water over the cutting area will help eliminate material chips and debris at the cutting surface. Multiple cuts were made per sample to ensure an approximate diameter of $10 \mathrm{~mm}$ and a thickness no greater than $3 \mathrm{~mm}$ to fit inside a microcorrosion cell. Figure 5.1 below shows represented sample from a retrieved hip implant. 


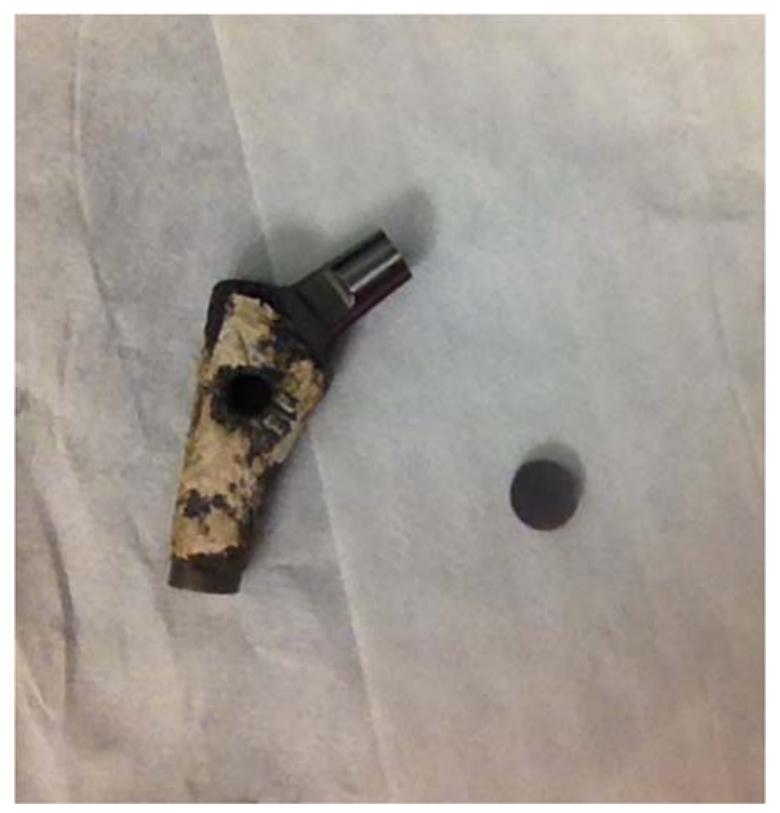

Figure 5.1: Sample Sectioned from Explanted Hip Prosthesis

Sectioned metallurgical samples were then placed in individual epoxy/resin molds to be used for mechanical grinding and polishing, figure 5.2. Silicon-carbide grit paper (Beuhler) attached to a rotating wheel disc was used to grind the metallurgical samples. Coarse grinding paper began at 120 and continued to 240, 320, 400, and 600. Fine grinding paper (p2400 and p4000) was then used to remove remaining surface scratches, figure 5.3. A continuous flow of water was incorporated into the grinding process to remove debris and prevent excessive heat generation. The samples were then polished with a polishing cloth and Alumina Oxide $\left(\mathrm{Al}_{2} \mathrm{O}_{3}\right)$ polishing paste. Samples were first polished with 1-micron $\mathrm{Al}_{2} \mathrm{O}_{3}$ particles then further polished to a mirror finish with 0.3 micron and 0.05 micron sized particles. Samples were then rinsed with water and dried in a stream of nitrogen gas. Samples were routinely inspected under an optical microscope to ensure that they were free of scratches and abrasive marks. 

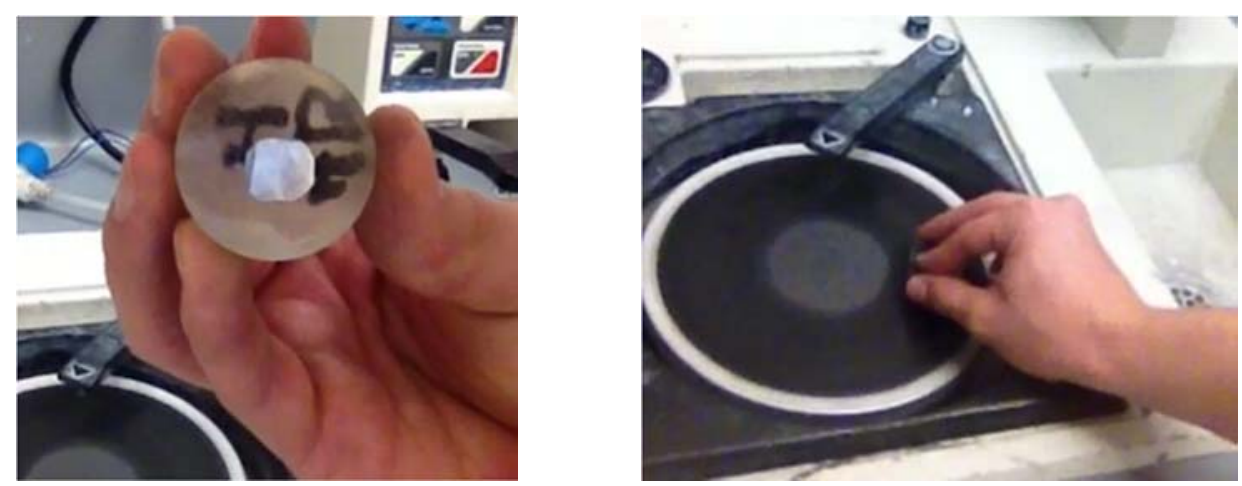

Figure 5.2: Metal Sample Mounted in Epoxy (left)

Figure 5.3: Manual Grinding of Metal Sample (right)

\subsection{Corrosion Test Set-up}

A treaded micro-corrosion glass tube was custom ordered from Ace Hardware along with a rubber O-ring, aluminum presser, a rubber treaded holder, carbon electrode and stopcork. Silver/silver chloride $(\mathrm{Ag} / \mathrm{AgCl})$ reference electrode was ordered from Warner Instruments. These parts were placed into an ultrasonic bath with deionized water for 15 minutes to remove residual debris. The sample was removed from the epoxy by placing in an incubator at $120^{\circ} \mathrm{C}$ for 40 minutes to allow the sample to be gently plucked out of the mold. Samples that were polished to a mirror finished were placed in an ultrasonic bath for 5 minutes with deionized water to remove any polishing powder/paste that may have become lodged into the sample. After cleaning, an O-ring was placed inside the micro-corrosion chamber. The metallurgical sample was placed above the O-ring. The aluminum rod held the metallurgical sample while the rubber fastener secured the aluminum rod to the micro-corrosion cell. This represents the base of the micro-corrosion cell, as illustrated in figure 5.4. The O-ring ensured that the electrolyte fluid would remain within the chamber of the test tube and that only the polished surface of the 
metallurgical sample was exposed to the solution. At the apex of the micro-corrosion cell, a stop-cork secures the $\mathrm{Ag} / \mathrm{AgCl}$ electrode while allowing the carbon electrode to pass through its through hole. It is important to ensure during set but that the $\mathrm{Ag} / \mathrm{AgCl}$ electrode and the carbon electrode are not in direct contact with each other.

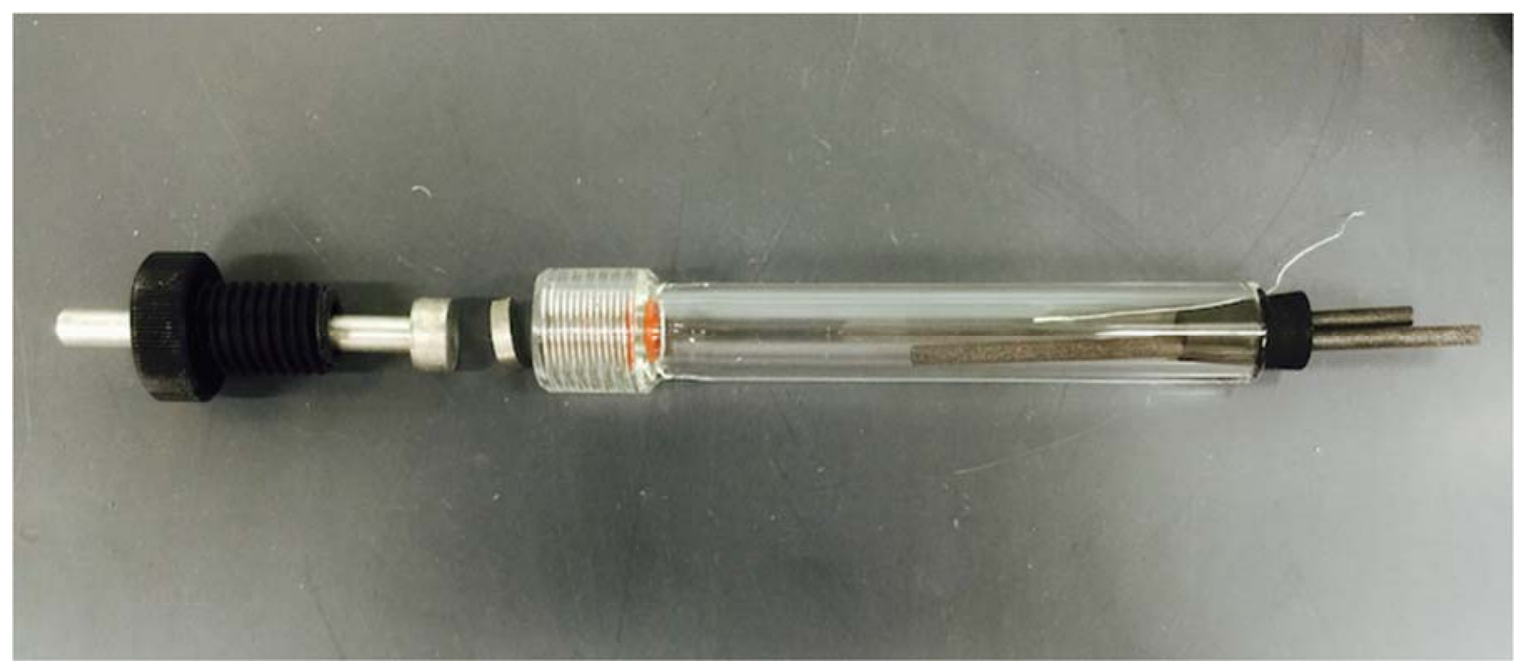

Figure 5.4 Assembly of Micro-corrosion Cell

Hanks Solution (Life Technologies) was selected as the electrolyte solution for accelerated corrosion testing. A list of the inorganic salts and their concentration in Hank's Solution can be found in table 5.1. Hank's Solution can be used to simulate physiological fluid; however, it lacks the biomolecules typically found in physiological fluid. Figure 5.5 provides an estimation of ionic and biomolecule species in blood plasma, interstitial fluid, and intracellular fluid. Physiologic fluid can be divided between intracellular fluid and extracellular fluid. Intracellular fluid is that fluid found inside a cell. All body fluids outside of cells are termed extracellular fluid. Blood plasma is the solution that suspends blood cells. Interstitial fluid is the solution that bathes the cells of 
tissue. Transcellular fluid is a small portion of extracellular fluid in unique locations.

Examples include cerebrospinal fluid, ocular fluid, and joint fluid.

\section{Inorganic Salts}

Calcium Chloride $(\mathrm{CaCl} 2)$ (anhyd.)

Magnesium Chloride ( $\mathrm{MgCl} 2-6 \mathrm{H} 2 \mathrm{O})$

Magnesium Sulfate (MgSO4-7H2O)

Potassium Chloride $(\mathrm{KCl})$

Potassium Phosphate monobasic (KH2PO4)

Sodium Bicarbonate (NaHCO3)

Sodium Chloride $(\mathrm{NaCl})$

Sodium Phosphate dibasic (Na2HPO4) anhydrous

D-Glucose (Dextrose)
Molecular Weight Concentration (mg/L) $\mathbf{m M}$

$\begin{array}{ccc}111 & 140 & 1.26 \\ 203 & 100 & 0.49 \\ 246 & 100 & 0.41 \\ 75 & 400 & 5.33 \\ 136 & 60 & 0.44 \\ 84 & 350 & 4.17 \\ 58 & 8000 & 137.93 \\ 142 & 48 & 0.34 \\ 180 & 1000 & 5.56\end{array}$

Table 5.1: Chemical Composition of Hank's Solution

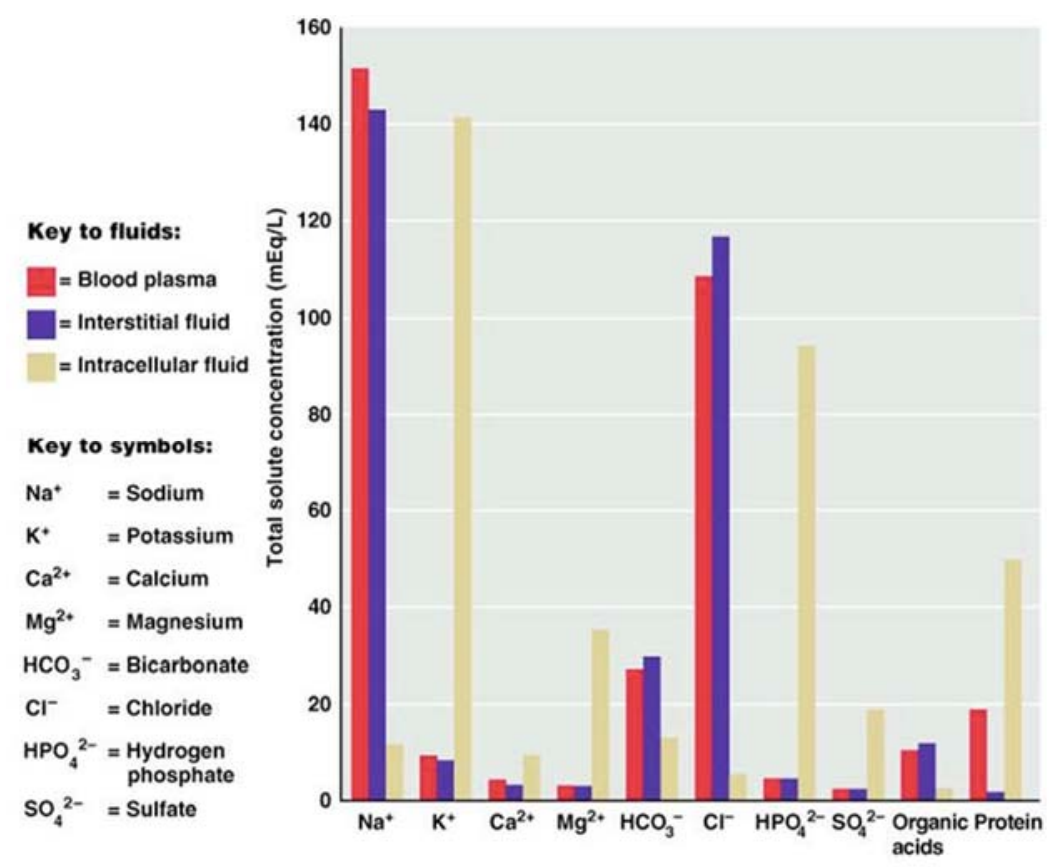

Figure 5.5: Chemical Composition of Body Fluids [100]

Aliquots of Hank's Solution are poured into $50 \mathrm{~mL}$ conical tubes for easy of use and transportation. These aliquots are placed inside an incubator to warm up to $37^{\circ} \mathrm{C}$. About $3-4 \mathrm{~mL}$ of Hank's Solution was poured into the micro-corrosion test tube. Once the microcorrosion cell was set up, the micro-corrosion cell was placed inside the incubator. A 
counter electrode (red alligator clip) within the incubator was connected to the carbon electrode. The reference electrode (white alligator clip) within the incubator was connected to the $\mathrm{Ag} / \mathrm{AgCl}$ electrode. The working electrode (green alligator clip) was within the incubator was connected to the aluminum rod. Figure 5.6 shows the color code combination when connecting the alligator clips to each electrode. Figure 5.7 shows the micro-corrosion cell properly set-up inside the incubator. The 3-electrode microcorrosion cell interphases with a potentiostat $\left(\mathrm{Gamry}^{\circledR}\right)$ to measure how the samples current density changes as a response to incremental change in electrode potential.

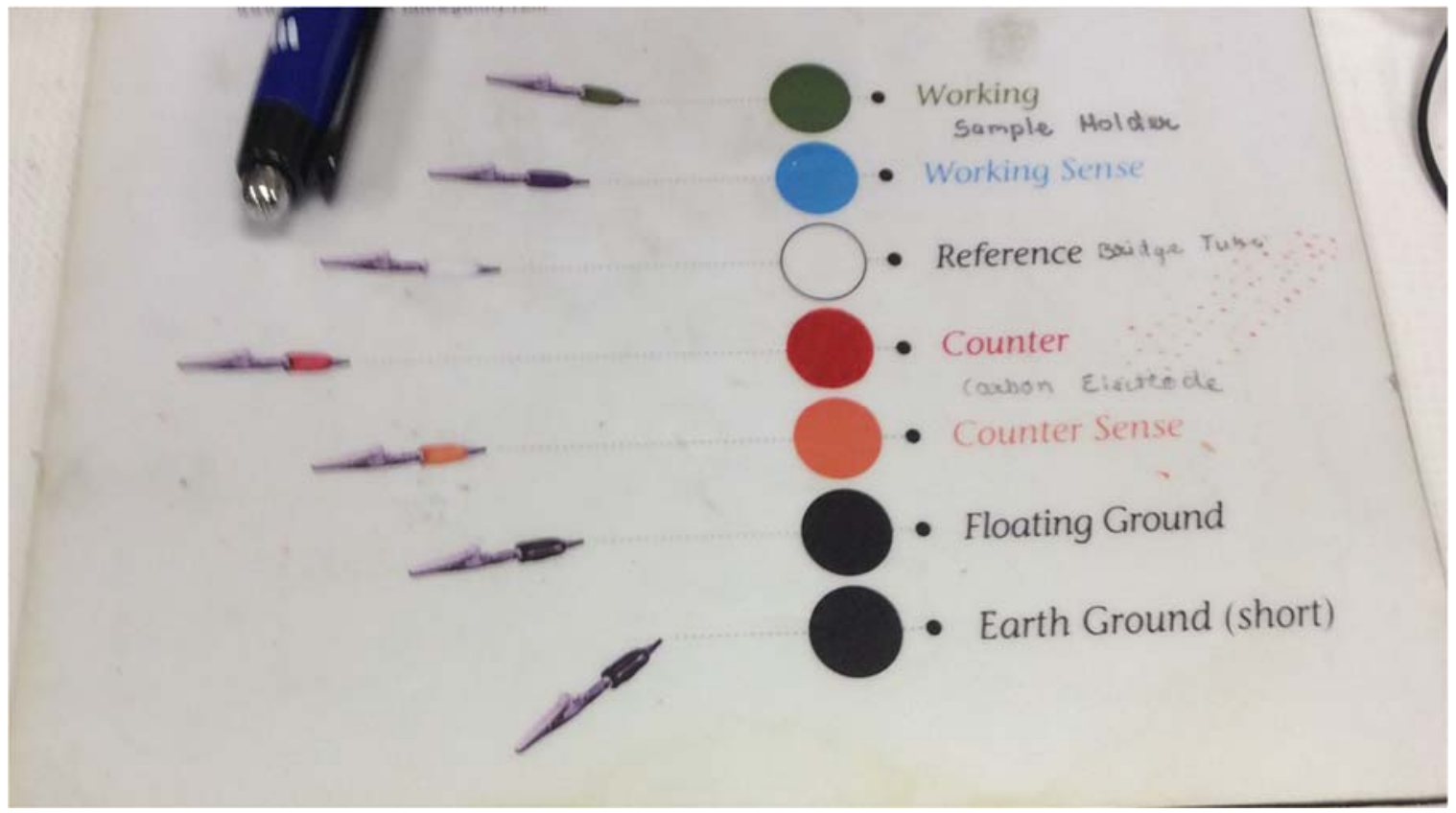

Figure 5.6: GAMRY Electrode Color Code

Once the micro-corrosion cell was properly setup inside the incubator, the incubator was allowed to reach a temperature equilibrium of $37^{\circ} \mathrm{C}$. Once the apparatus equilibrated, potentiodynamic cyclic polarization utilized DC voltage was implemented from $-1.5 \mathrm{~V}$ to $+1.5 \mathrm{~V}$ relative to open circuit potential. The scan was swept from cathodic (negative 
potential) to anodic (positive potential) at a scan rate of $1 \mathrm{mV} / \mathrm{s}$. Furthermore, the scan was reference at the apex to $+1 \mathrm{~V}$ relative to open circuit potential. Image 6 shows the accelerated corrosion apparatus inside the incubator and connected to the potentiostat.

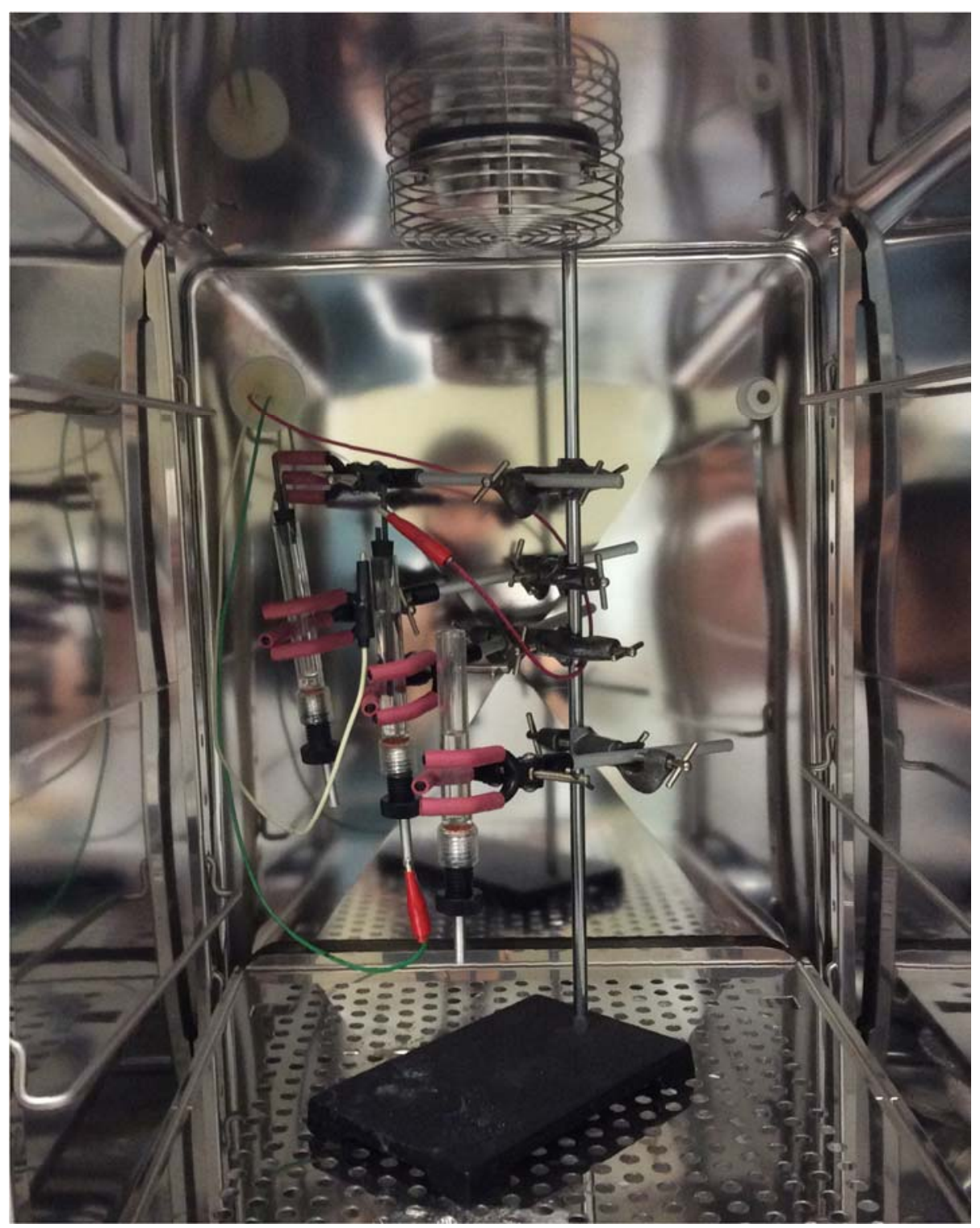

Figure 5.7: Micro-corrosion cell, inside incubator, connected to the Potentiostat 
Optical microscopy and scanning electron microscopy were used to study the surface integrity of the sample before and after corrosion. Furthermore, energy dispersive spectroscopy was utilized to study the chemical composition of the sample before and after corrosion. Corrosion data was obtained in the form of Tafel extrapolation of the polarization graphs generated from GAMRY analyst. 


\section{Chapter 6 Results}

\subsection{Microstructure Images Before and After Corrosion}

Low Carbon Wrought (LCW) CoCrMo Alloy Microstructure

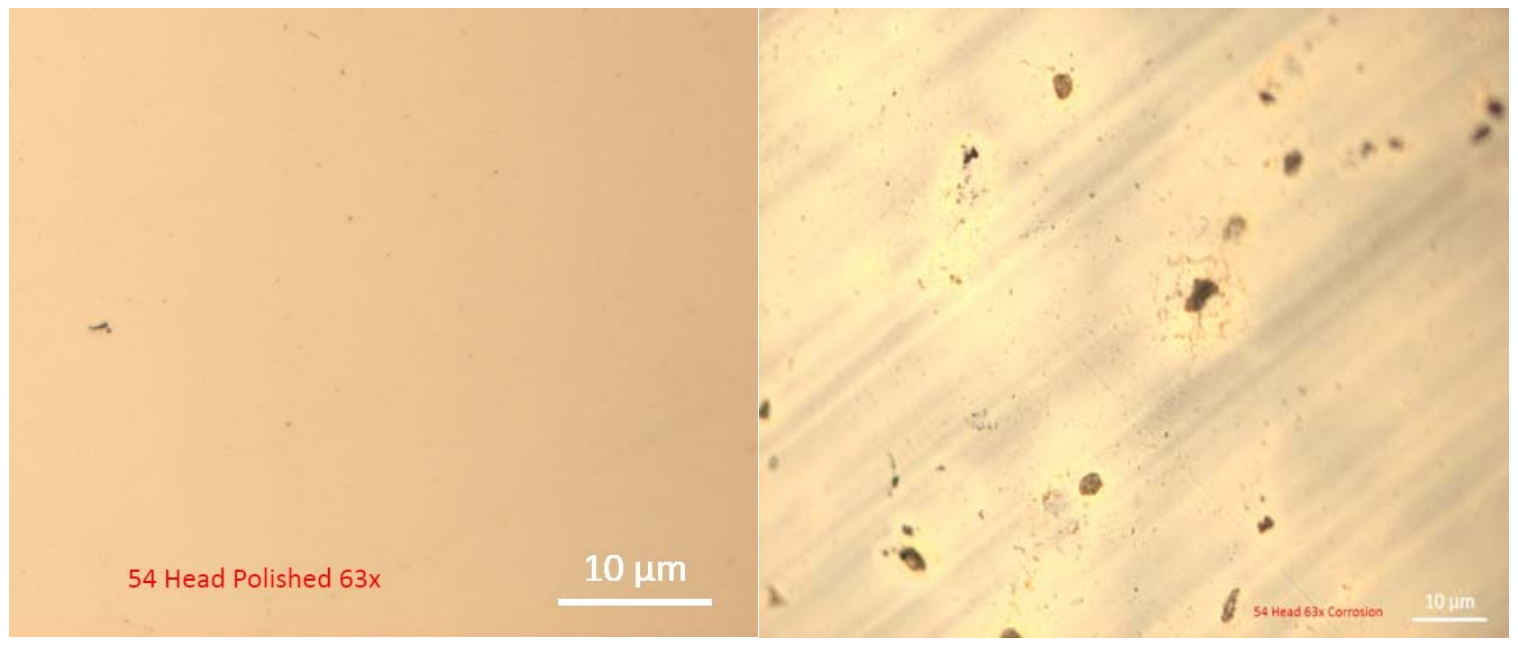

Figure 6.1: Polished Optical Micrograph of a Low Carbon Wrought Sample (left)

Figure 6.2: Corrosion Optical Micrograph of a Low Carbon Wrought Sample (right)

Figure 6.1 and 6.2 are optical micrographs at $63 \mathrm{x}$ magnification of a low carbon wrought alloy of the sample polished before corrosion and after corrosion, respectively. The purpose of polishing is to create a mirror finish on the surface of a metallurgical sample. Ideally, the surface should be free of defects such as scratches from the grinding process. A smooth mirror finish ensures that corrosion is more uniform along the surface. Figure 6.2 shows localized corrosion around pits, which also indicate more localized form of corrosion. One possible explanation is intergranular corrosion as a result of chromium depletion in this region. All optical microscope images in the results section were taken with a Carl Zeiss AxioCam (Germany) optical microscope. 


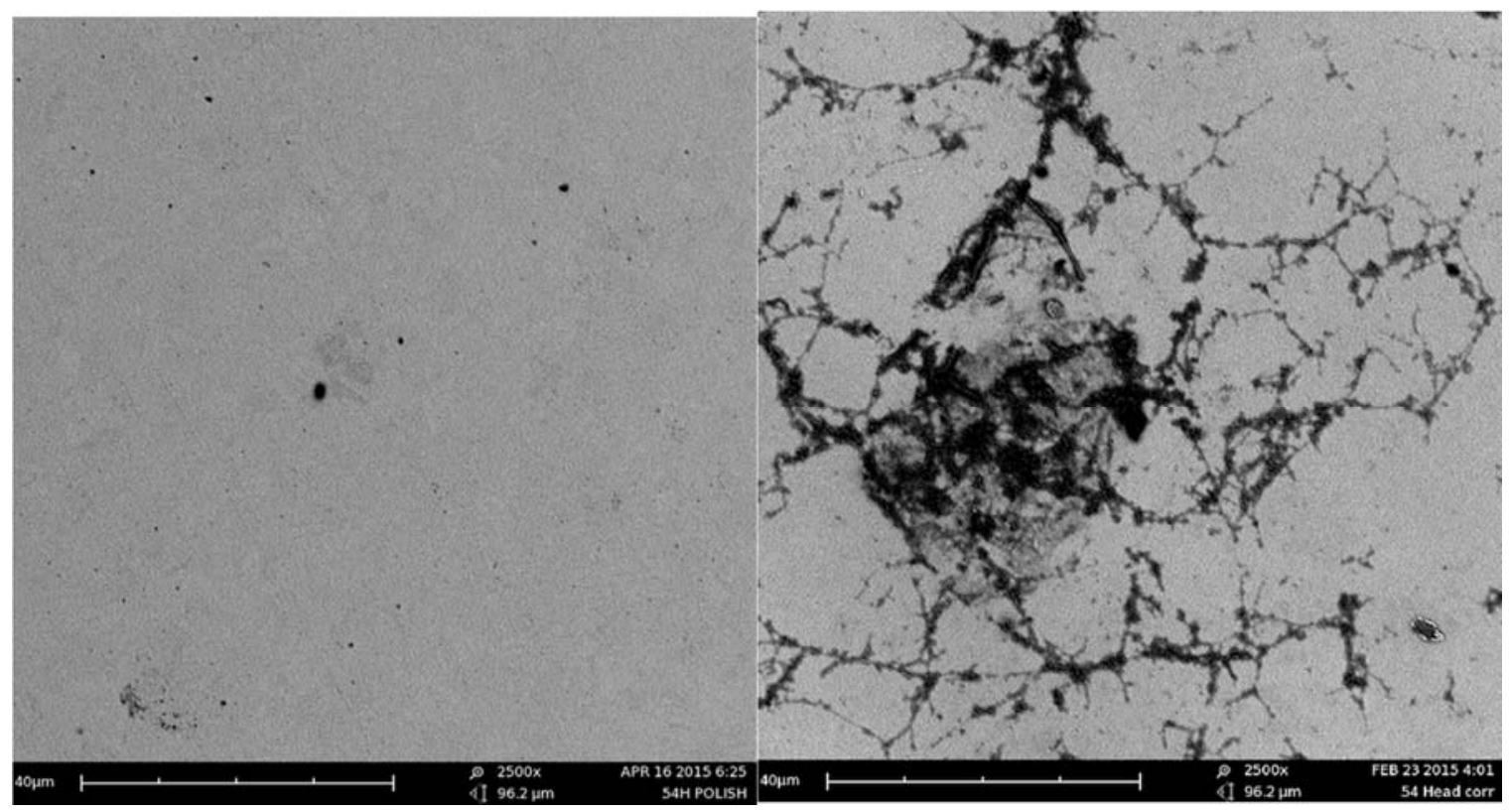

Figure 6.3: Polished SEM Micrograph of a Low Carbon Wrought Sample (left) Figure 6.4: Corrosion SEM Micrograph of a Low Carbon Wrought Sample (right)

Figure 6.3 and 6.4 are SEM images of the same sample in figure 6.1 and 6.2. These SEM images are at 2500x magnification. Figures 6.1-6.4 are from the same sample, which represents a low carbon, wrought CoCrMo alloy. Figure 6.4 is a higher magnification of one of the pits found in figure 6.2. All SEM images in the results section were obtained from a tabletop SEM (PhenomWorld, USA) with an accelerating voltage of $5 \mathrm{KeV}$. 


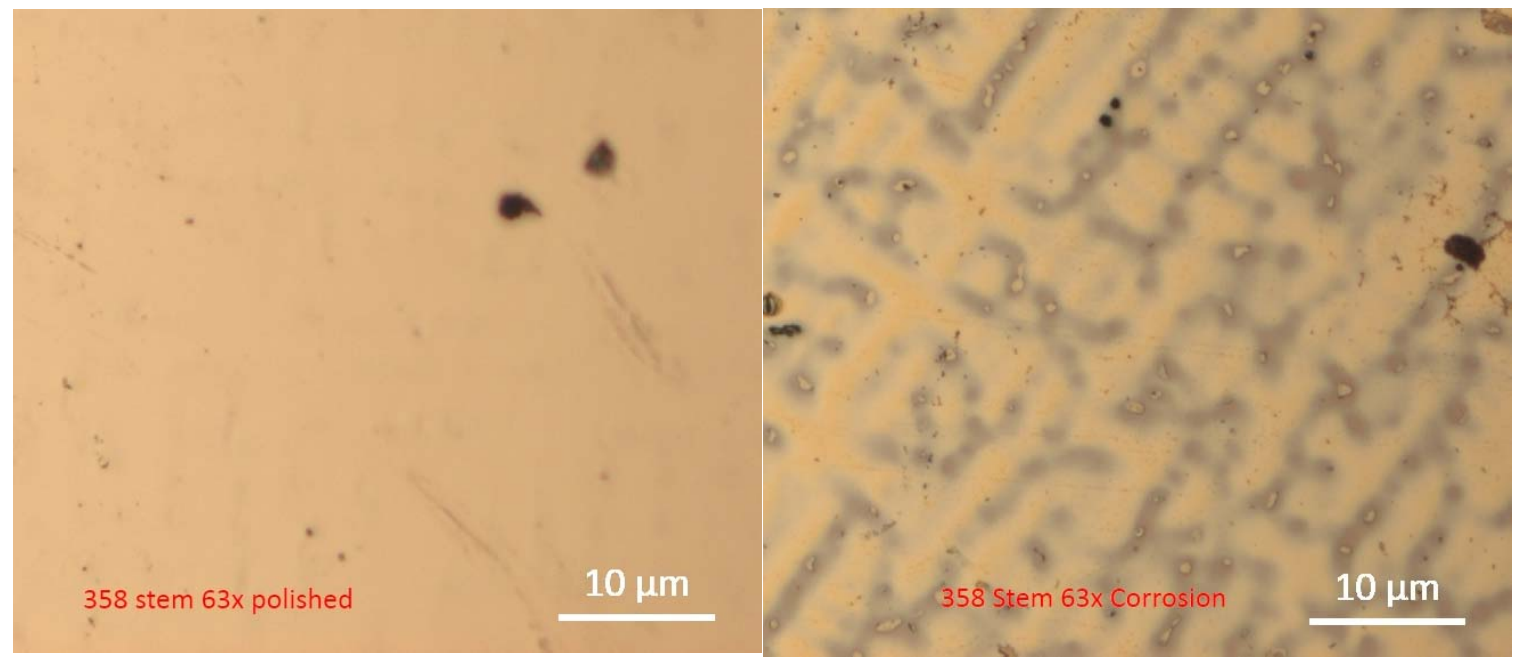

Figure 6.5: Polished Optical Micrograph of an As-Cast Sample (left) Figure 6.6: Corrosion Optical Micrograph of an As-Cast Sample (right)

Figures 6.5 and 6.6 are optical micrographs at $63 \mathrm{x}$ magnification of an as-cast alloy of the sample polished before corrosion and after corrosion, respectively. Figure 6.6 shows the characteristic dendritic microstructure of as-cast alloys. As-cast processing results in a heterogeneous chemical composition because of the fast cooling rates that result in a second phase precipitating out of a first phase. Generally the one of the alloying elements ( $\mathrm{Co}, \mathrm{Cr}$ or $\mathrm{Mo}$ ) with the higher liquidus point solidifies first. It is evident that the microstructures of as-cast alloys have large patterns observable under low magnification. 


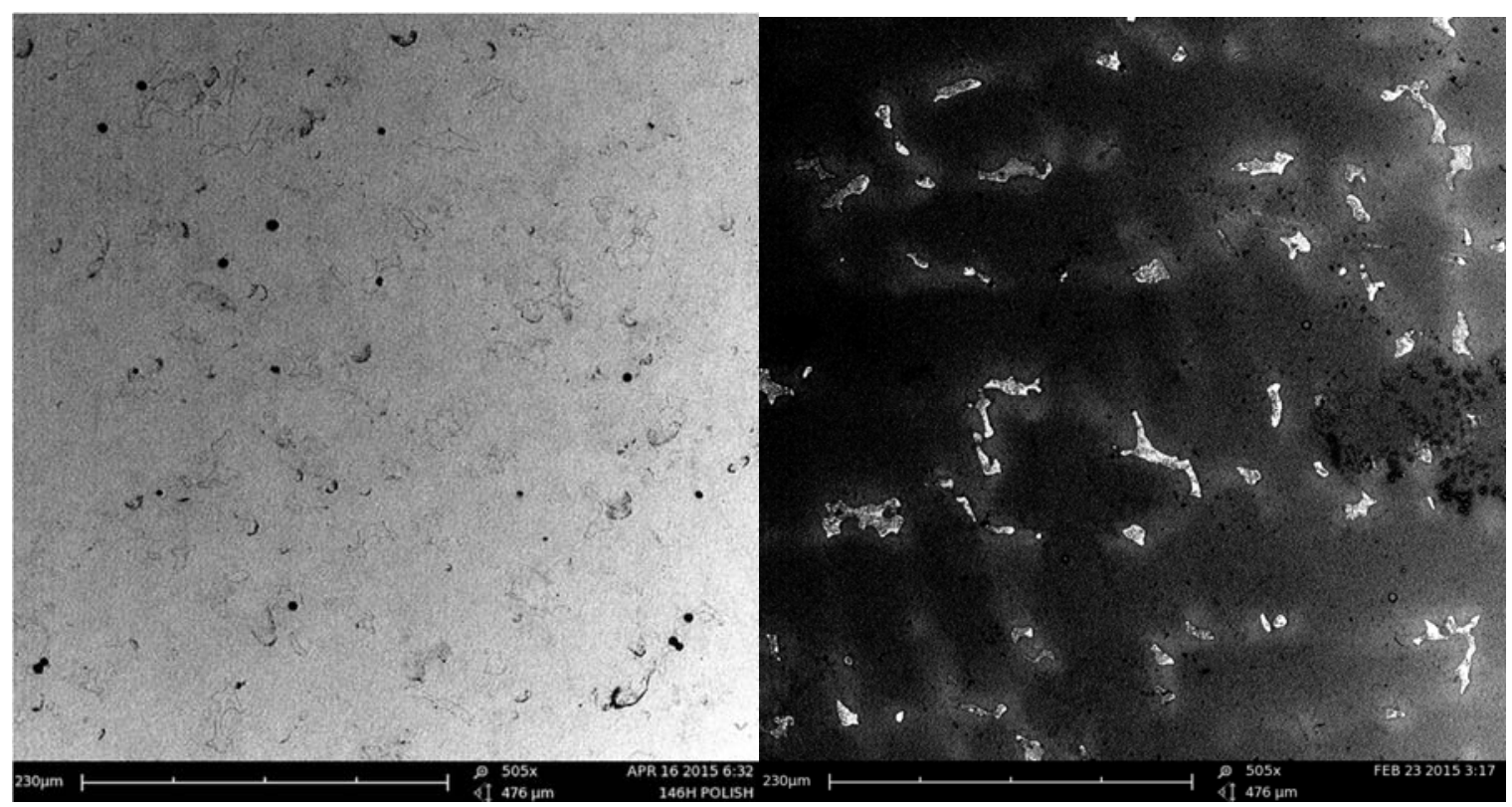

Figure 6.7: Polished SEM Micrograph of an As-Cast Sample (left)

Figure 6.8: Corrosion SEM Micrograph of an As-Cast Sample (right)

Figures 6.7 and 6.8 are scanning electron micrographs at 505x magnification of an as-cast alloy of a sample polished before corrosion and after corrosion respectively.

High Carbon Wrought CoCrMo Alloy Microstructure with Large Grains

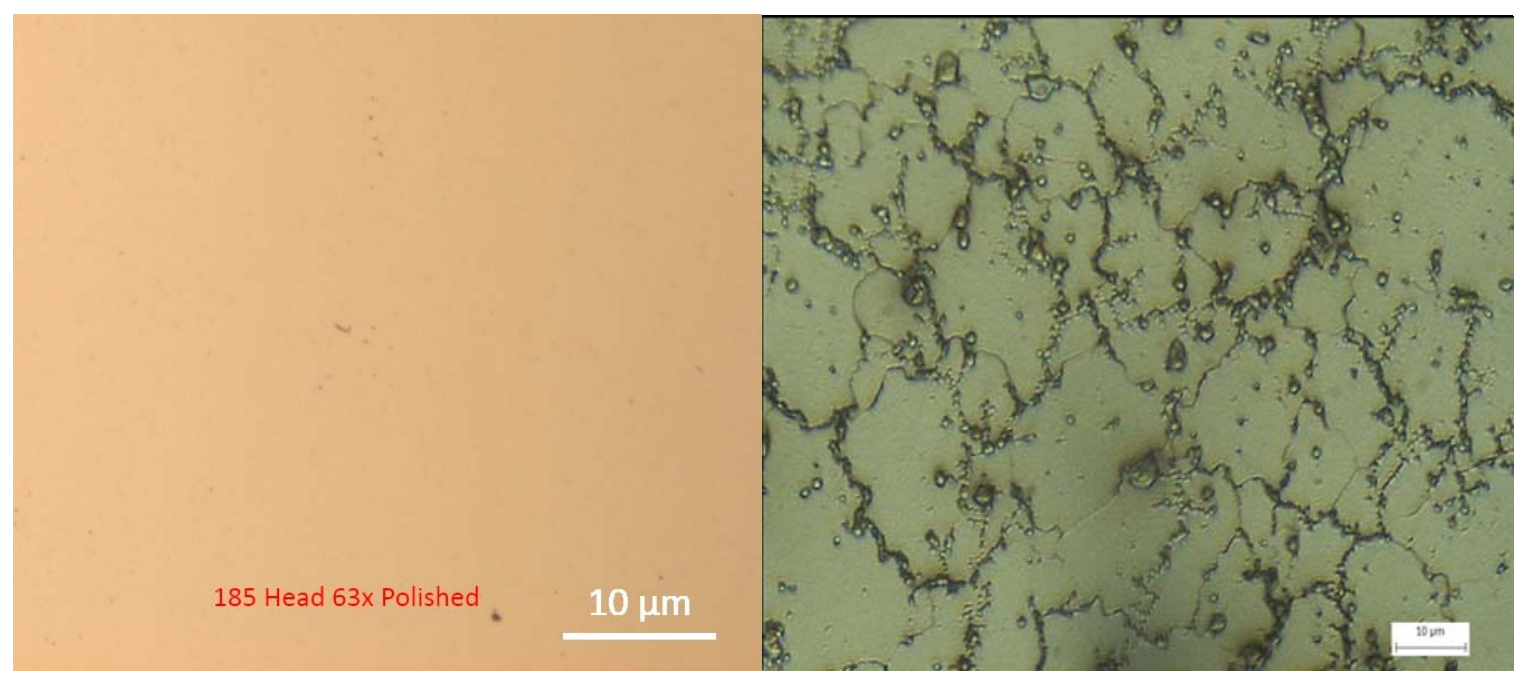

Figure 6.9: Polished Optical Micrograph of a High Carbon Wrought Sample (left) Figure 6.10: Corrosion Optical Micrograph of a High Carbon Wrought Sample (right) 
Figures 6.9 and 6.10 are the optical micrographs of a high carbon wrought CoCrMo alloy larger average grain diameters. Figure 6.9 is an optical micrographs at $63 \mathrm{x}$ magnification while image 6.10 is a shows the sample after corrosion at 500x magnification.

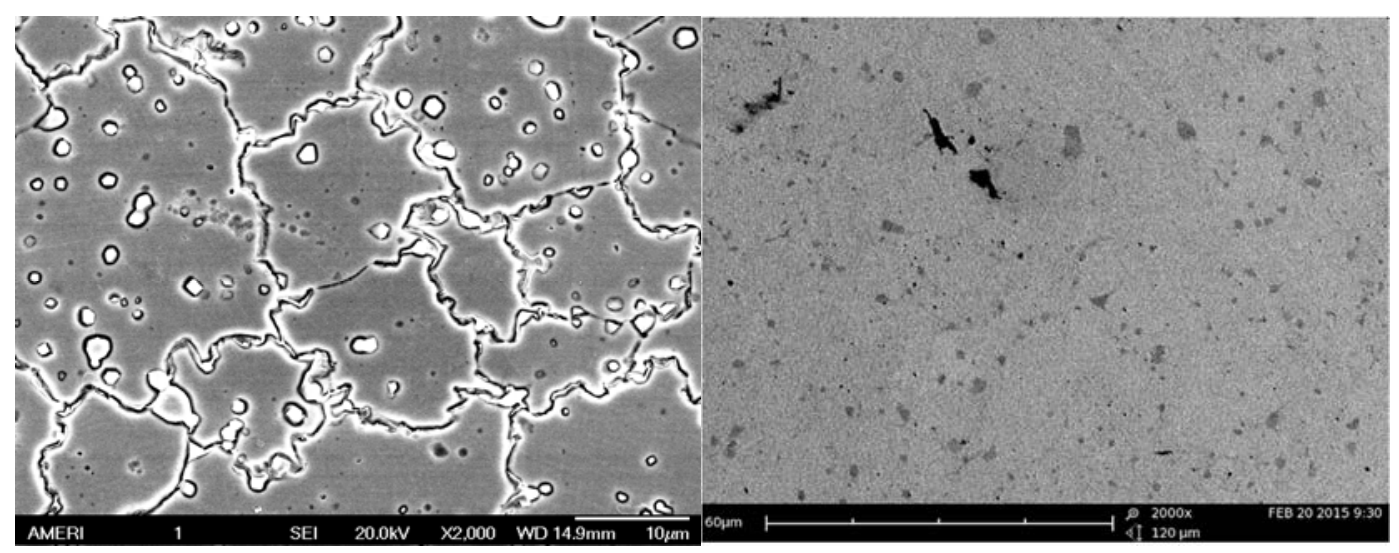

Figure 6.11: Polished SEM Micrograph of a High Carbon Wrought Sample (left) Figure 6.12: Corrosion SEM Micrograph of a High Carbon Wrought Sample (right)

Figures 6.11 and 6.12 are scanning electron micrographs at 2000x magnification of a high carbon wrought CoCrMo alloy of a sample before and after corrosion, respectively. This is the same sample as found in figures 6.9 and 6.10. It is evident that the wrought sample has significantly smaller microstructure than the as-cast sample as represented by figure 6.8. The grain boundaries are evident by the black outline surrounding the grey grains. During the solidification process, grains grow at different directions and orientations. When separate grains begin to consolidate the mismatch in direction and orientation creates high-energy sites, known as grain boundaries. Grain boundaries are a higher energy state than grains and subsequently more reactive relative to grains during chemical attack. This sample has secondary phase white precipitation formation both within the grains and at the grain boundaries. The grains have an approximate diameter of $20 \mu \mathrm{m}$ (estimated diameter range of $10-30 \mu \mathrm{m}$ ). The white precipitates have an 
approximate diameter of $1 \mu \mathrm{m}$ within the grains and with a range of $0.5 \mu \mathrm{m}$ to $2 \mu \mathrm{m}$ at both the grain boundaries and inside the grains.

High Carbon Wrought CoCrMo Alloy Microstructure with Small Grains

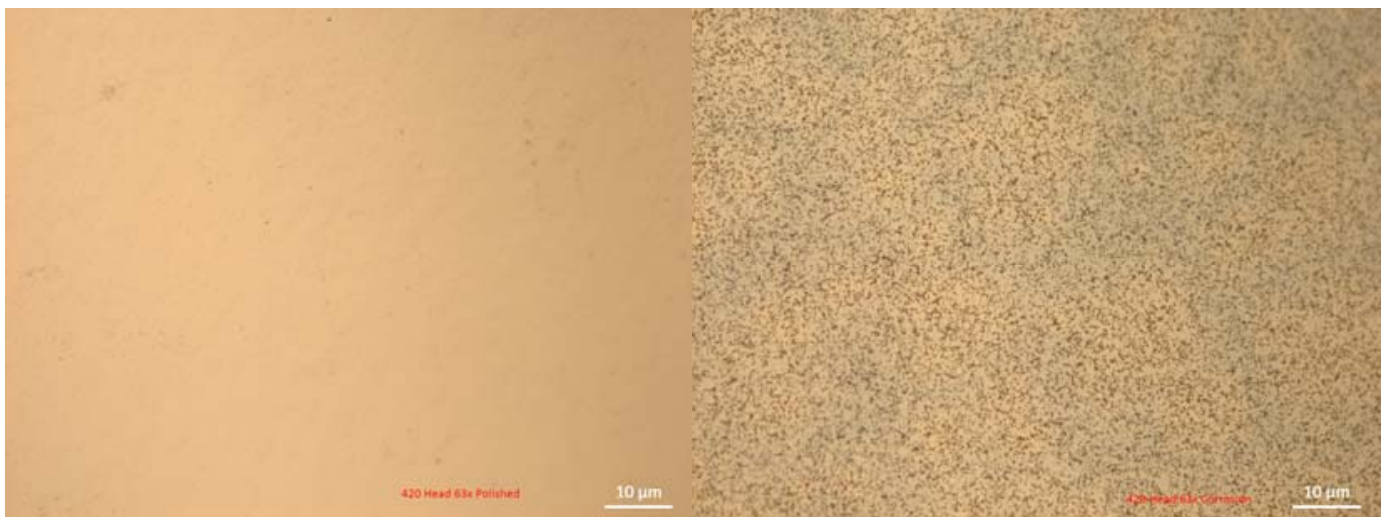

Figure 6.13: Polished Optical Micrograph of a High Carbon Wrought Sample Fine Grains (left)

Figure 6.14: Corrosion Optical Micrograph of a High Carbon Wrought Sample Fine Grains (right)

Figures 6.13 and 6.14 are optical micrographs at $63 \mathrm{x}$ magnification of a low carbon wrought alloy of the sample polished before corrosion and after corrosion, respectively. 


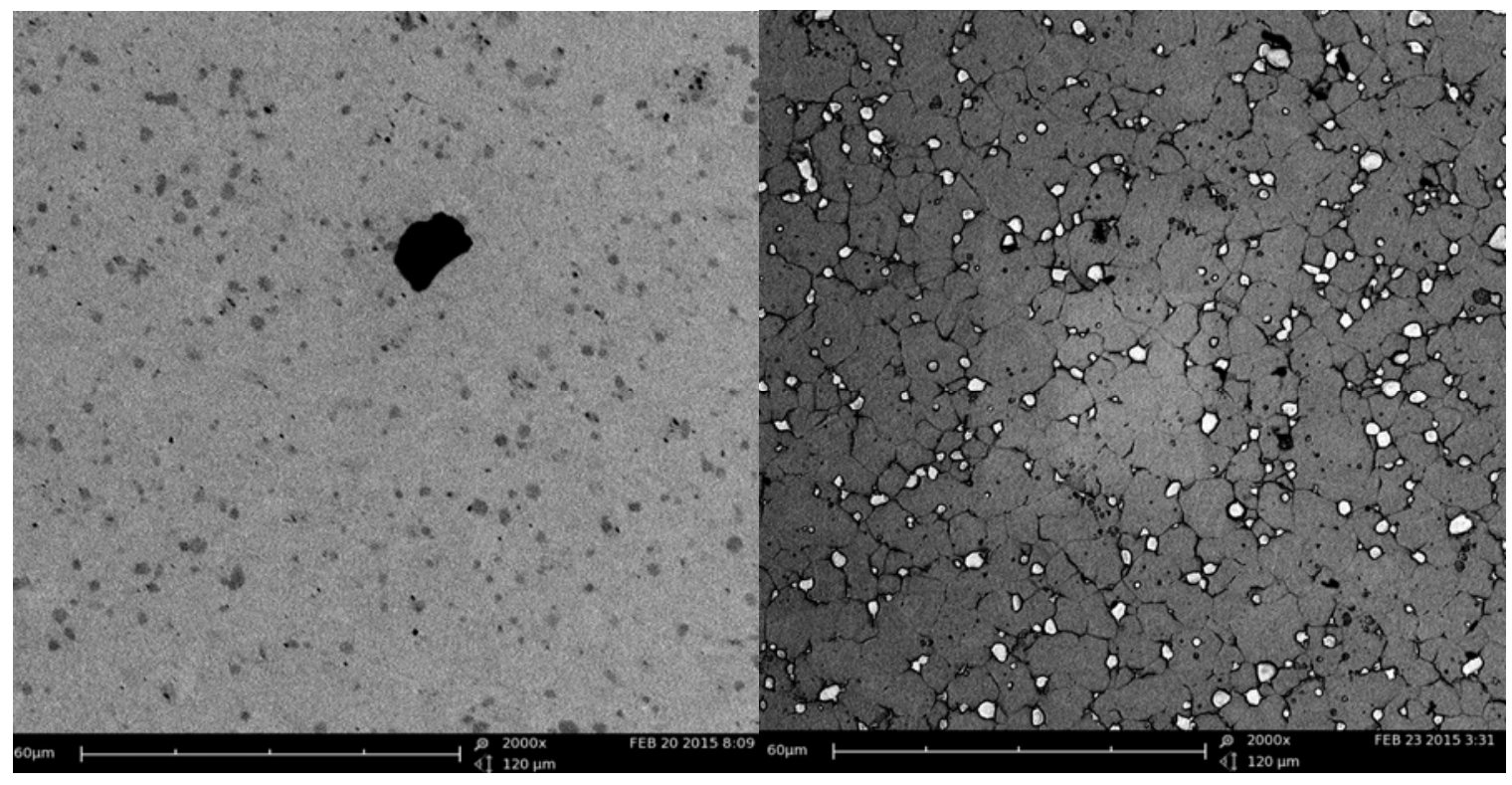

Figure 6.15: Polished SEM Micrograph of a High Carbon Wrought Sample Fine Grains (left)

Figure 6.16: Corrosion SEM Micrograph of a High Carbon Wrought Sample Fine Grains (right)

Figures 6.15 and 6.16 are scanning electron micrographs at 2000x magnification of a high carbon wrought alloy with small grains. It is important to realize that figures 6.96.12 are a different sample than figures 6.13-6.16 - even though both are classified as wrought high carbon CoCrMo. The first noticeable difference is that the average grain diameters in figure 6.16 are approximately $7 \mu \mathrm{m}(4 \mu \mathrm{m}-10 \mu \mathrm{m})$. These grains are smaller than the grains found in figure 6.12. The secondary white precipitates have a size of around $1.5 \mu \mathrm{m}$ with a range of $1-2 \mu \mathrm{m}$. Second, the secondary white phase precipitates only at the grain boundary. The different microstructures arise from different processing techniques. It is well established that forging (imparting thermal and mechanical strains) can reduce the grain size. It may be quite possible that the sample from figures $6.13-6.16$ went through a forging processing after wrought production whereas the sample in images did not. 


\subsection{Energy Dispersive Spectroscopy}

Electron dispersive spectroscopy (EDS) utilized a high-energy focused electron beam to emit an X-ray spectrum of a solid sample. EDS is able to identify the chemical composition that results from the contrast topography differences on an SEM image. Precision obtained from samples is limited by statistical error. For major alloying samples analytical accuracy is between $\pm 1-2 \%$. EDS was scanned on several different polished samples. Figure 6.17 shows three EDS point analysis taken per sample. Figure 6.18 shows the EDS spectrum of elements found in CoCrMo alloys. For simplicity and to avoid chemical analysis error only cobalt, chromium, and molybdenum where quantified because each element makes up more than 5 weight percent in the alloy. Chemical compositions from the EDS analysis can be found in table 6.1.

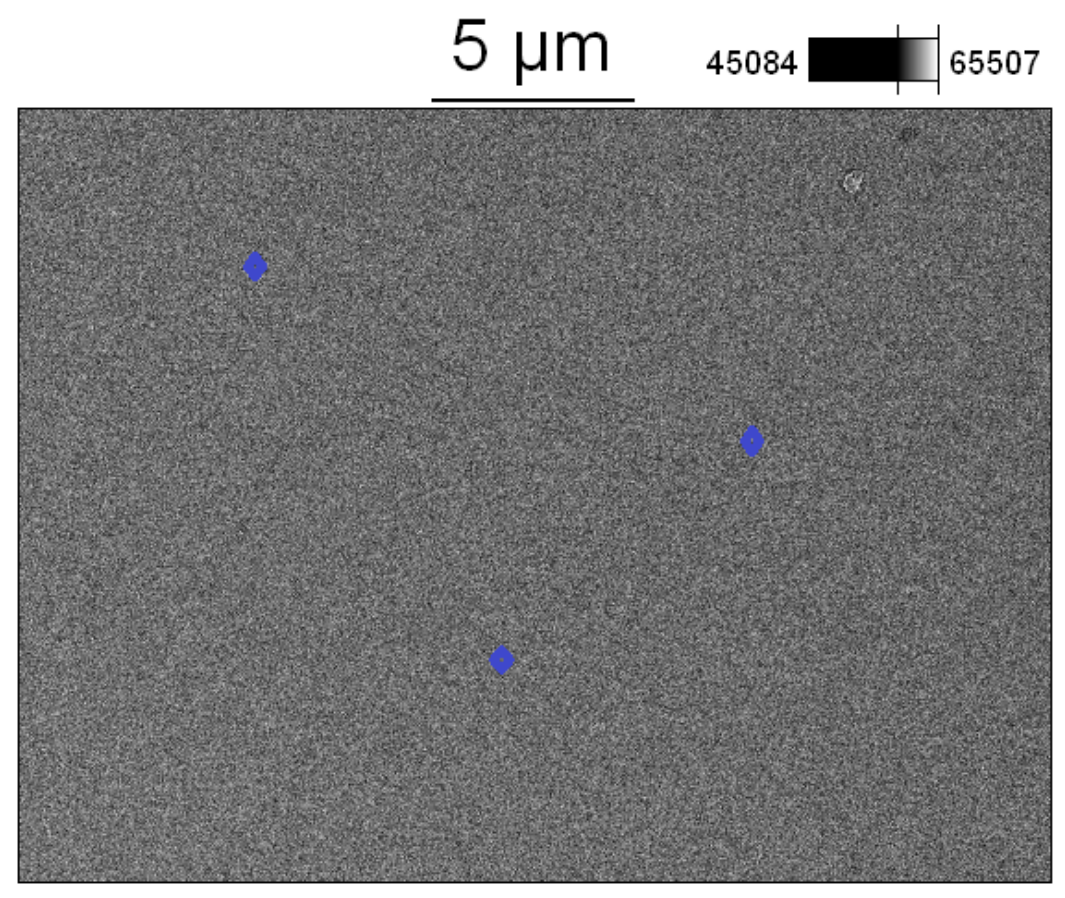

Figure 6.17: EDS Scan of a polished sample 


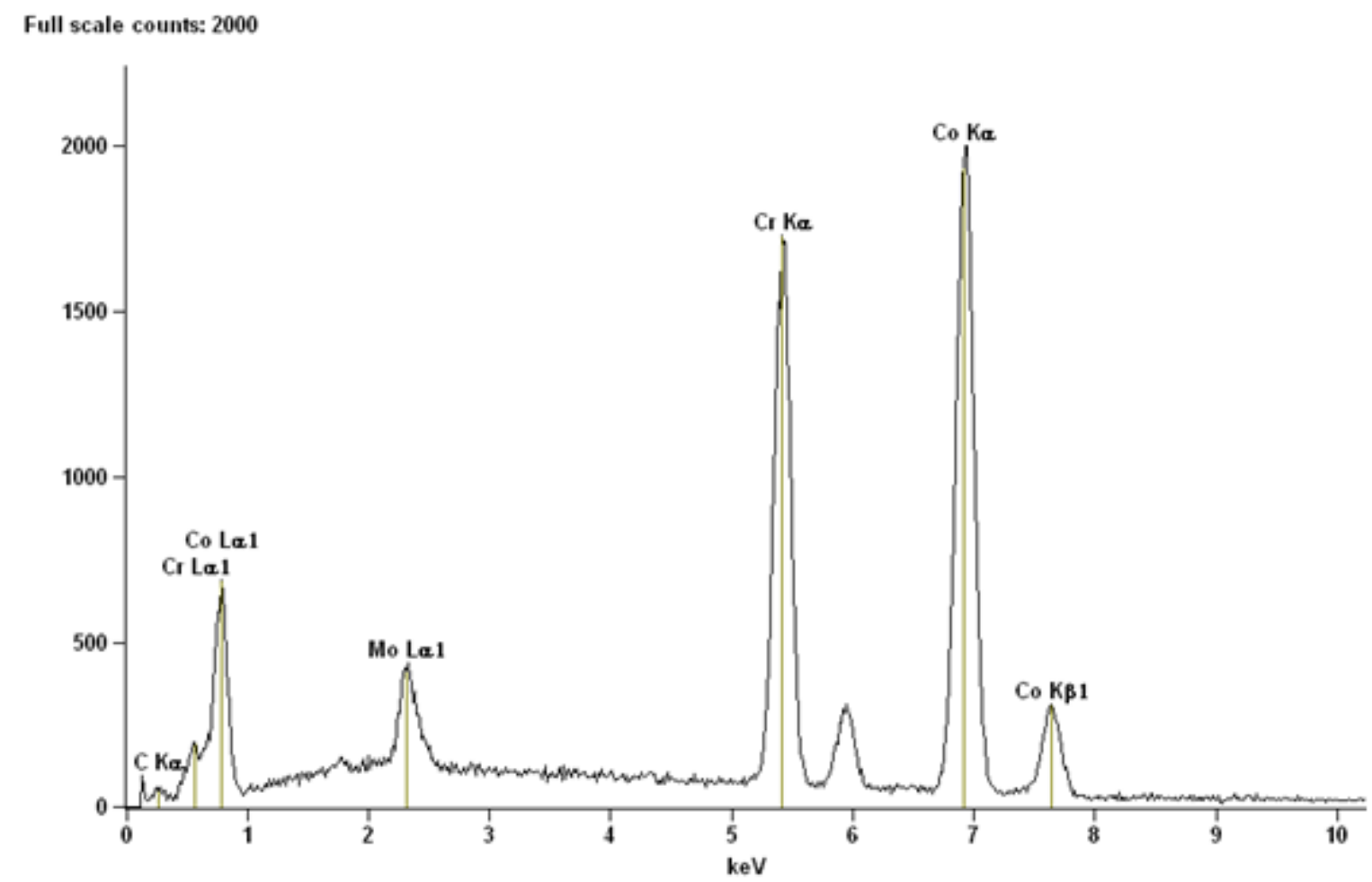

Figure 6.18: Representative EDS analysis of a polished sample

\begin{tabular}{|c|c|c|c|}
\hline \multicolumn{4}{|c|}{ Weight Percentage } \\
\hline Sample & Cobalt & Chromium & Molybdenum \\
\hline Wrought LC & 63.55 & 28.12 & 5.45 \\
\hline Wrought LC & 69.07 & 24.65 & 6.28 \\
\hline Wrought HC & 60.34 & 31.75 & 7.91 \\
\hline Wrought LC & 62.67 & 30.58 & 6.42 \\
\hline As-Cast & 59.72 & 31.47 & 8.81 \\
\hline Wrought HC & 63.92 & 29.77 & 5.28 \\
\hline Wrought LC & 65.80 & 26.94 & 4.47 \\
\hline Wrought LC & 69.70 & 24.65 & 5.65 \\
\hline Wrought HC & 62.31 & 27.12 & 7.17 \\
\hline Average & 64.12 & 28.34 & 6.38 \\
\hline St. Deviation & 3.50 & 2.72 & 1.38 \\
\hline
\end{tabular}

Table 6.1: Chemical analysis of figure 6.18 
Table 6.1 represents the EDS point scan of different CoCrMo samples after they were polished. Each sample represents a different component of an explanted retrieved hip prosthesis. Averages for each sample were not taken; however, table 6.1 provides both the average and standard deviation for the group. Both ASTM F75 and F1537 provide chemical weight percentage range for each element found in the CoCrMo alloys approved for use in orthopedic implants. Although minor alloying elements have at most $1 \%$ by weight in the alloy, it was preferred not to include elements in the EDS analysis. Elements such as carbon play an important role in the microstructure and material properties, such as hardness and strength; using EDS to measure carbon content can lead to erroneous estimates. Thereby, it was determined to conduct EDS analysis exclusively of the cobalt, chromium, and molybdenum. From both ASTM standards, chromium has a 26 to 30 -weight percentage, while molybdenum has a 5 to 7 weight percentage. From table 6.1, the average weight percentage is 64.12 for cobalt, 28.34 for chromium, and 6.38 for molybdenum. The average chemical composition of the samples is acceptable to the standards provided by both ASTM F75 and F1537.

\section{EDS After Corrosion}

Figures 6.19-6.21 show EDS point analysis of three microstructures. The EDS analyses of figures 6.19-6.21 are taken at 5000x magnification with an accelerating voltage of $20 \mathrm{KeV}$. The chemical composition of the grains and secondary phases were analyzed 3 times for each sample. The blue points represent EDS analysis of the secondary white phases. The representative EDS spectrum of the secondary white phase is found in figure 6.22. The chemical composition was recorded in table 5.2. The red points represent EDS 
analysis of the grains. The representative EDS spectrum of the grains is found in figure 6.23 with the chemical composition recorded in table 5.2.

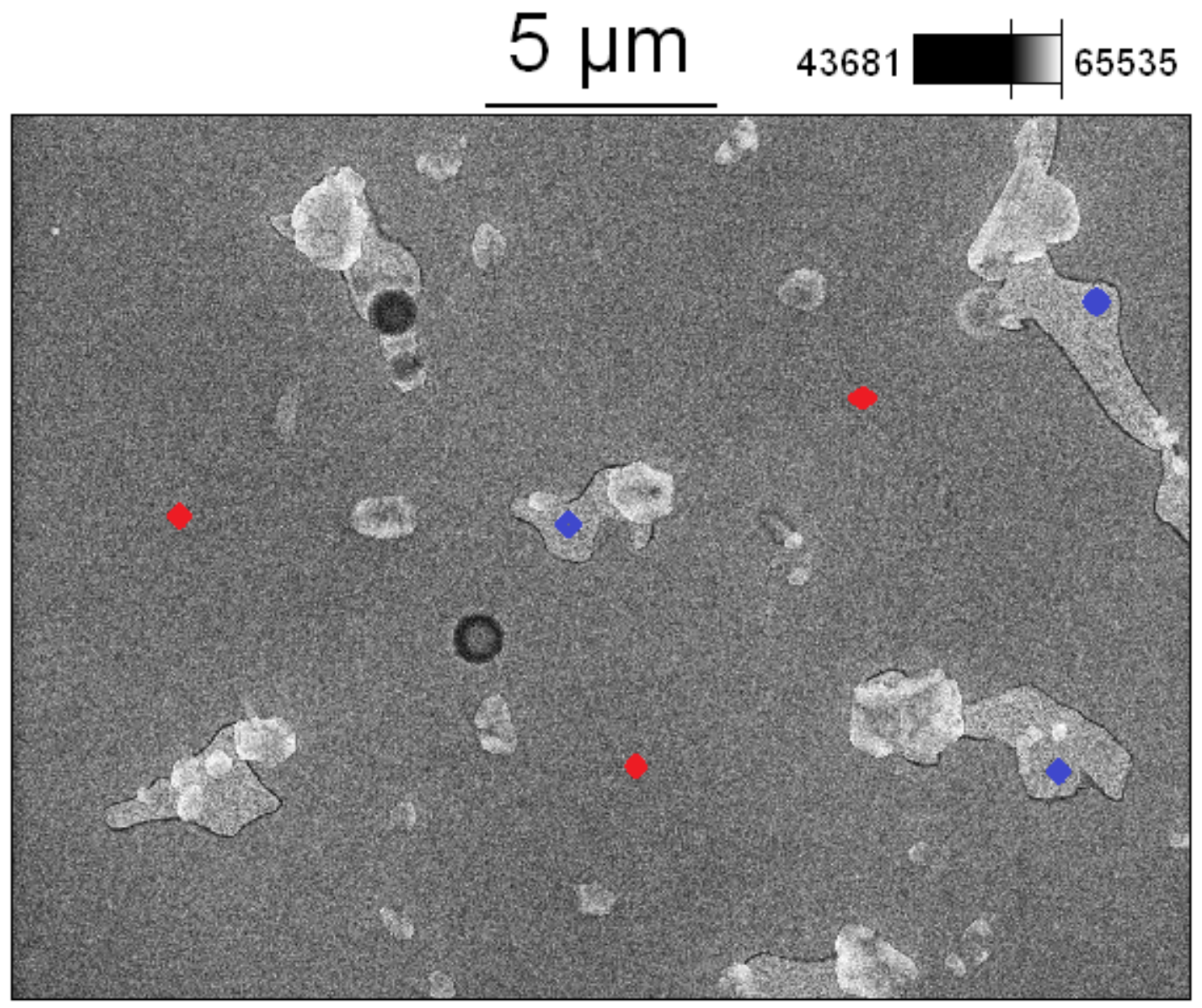

Figure 6.19: EDS point scans of an As-Cast CoCrMo Alloy after corrosion 


\begin{tabular}{|c|c|c|c|}
\hline \multicolumn{4}{|c|}{ Weight Percentage } \\
\hline As-Cast & Cobalt & Chromium & Molybdenum \\
\hline Red 1 & 50.00 & 26.95 & 23.05 \\
\hline Red 2 & 49.19 & 27.75 & 23.06 \\
\hline Red 3 & 46.86 & 32.07 & 21.08 \\
\hline Average & 48.68 & 28.92 & 22.40 \\
\hline St. Dev & 1.63 & 2.75 & 1.14 \\
\hline Blue 1 & 28.73 & 67.21 & 4.05 \\
\hline Blue 2 & 28.98 & 66.37 & 4.65 \\
\hline Blue 3 & 28.12 & 67.94 & 3.95 \\
\hline Average & 28.61 & 67.17 & 4.22 \\
\hline St. Dev & 0.44 & 0.79 & 0.38 \\
\hline
\end{tabular}

Table 6.2: Chemical Analysis of figure 6.19

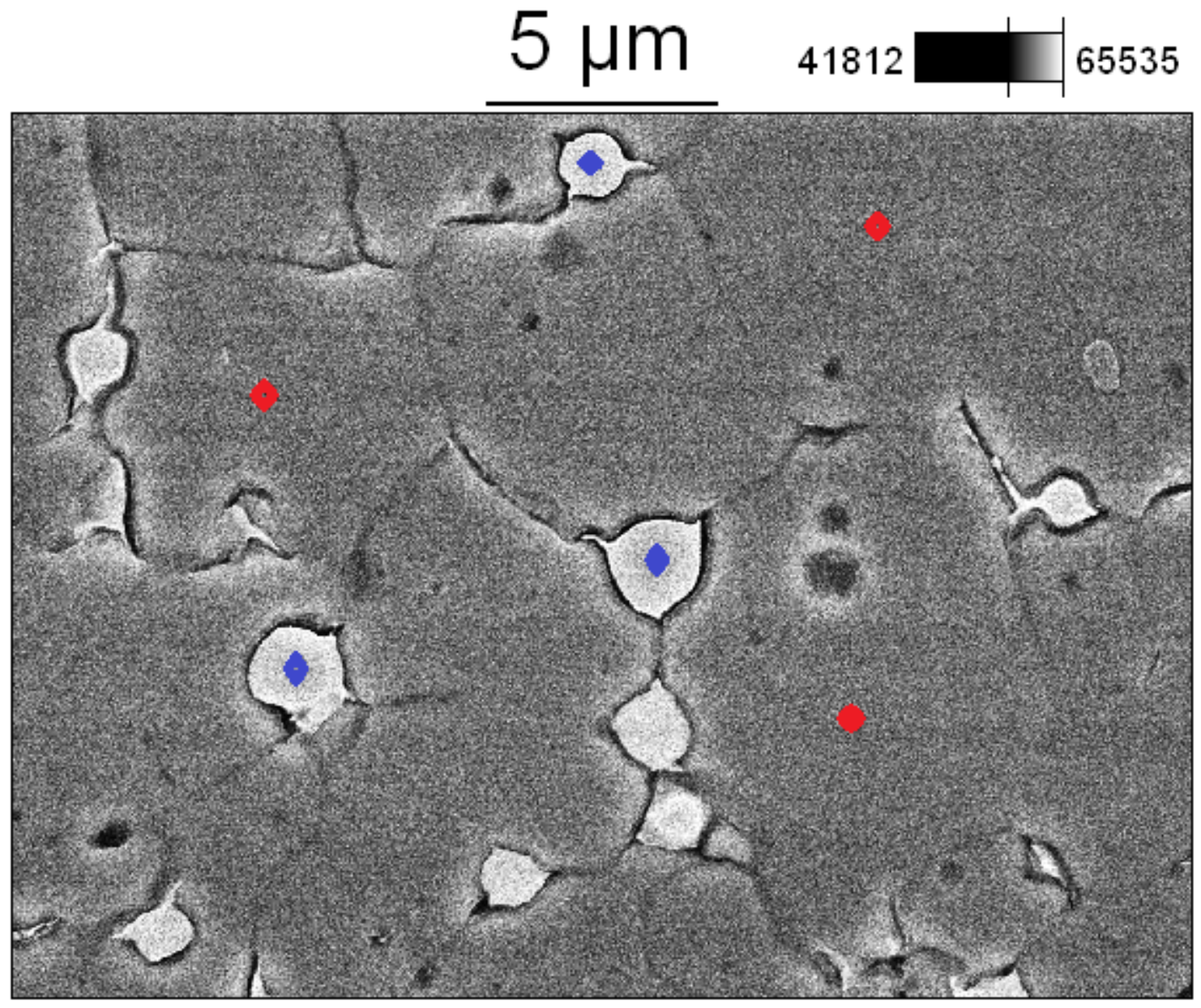

Figure 6.20: EDS Point Scans of High Carbon Wrought (fine grains) CoCrMo alloy after corrosion 


\begin{tabular}{lrrr}
\multicolumn{4}{c}{ Weight Percentage } \\
HC Wrought & $\begin{array}{r}\text { Cobalt } \\
\text { Chromium }\end{array}$ & Molybdenum \\
Red 1 & 70.42 & 16.98 & 12.60 \\
Red 2 & 70.71 & 16.68 & 12.61 \\
Red 3 & 66.96 & 21.31 & 11.73 \\
Average & 69.36 & 18.32 & 12.31 \\
St Dev & 1.70 & 2.12 & 0.41 \\
Blue 1 & 29.15 & 64.62 & 6.23 \\
Blue 2 & 28.75 & 64.94 & 6.31 \\
Blue 3 & 28.53 & 65.38 & 6.09 \\
Average & 28.81 & 64.98 & 6.21 \\
St Dev & 0.31 & 0.38 & 0.11
\end{tabular}

Table 6.3: EDS Chemical Analysis of figure 6.20

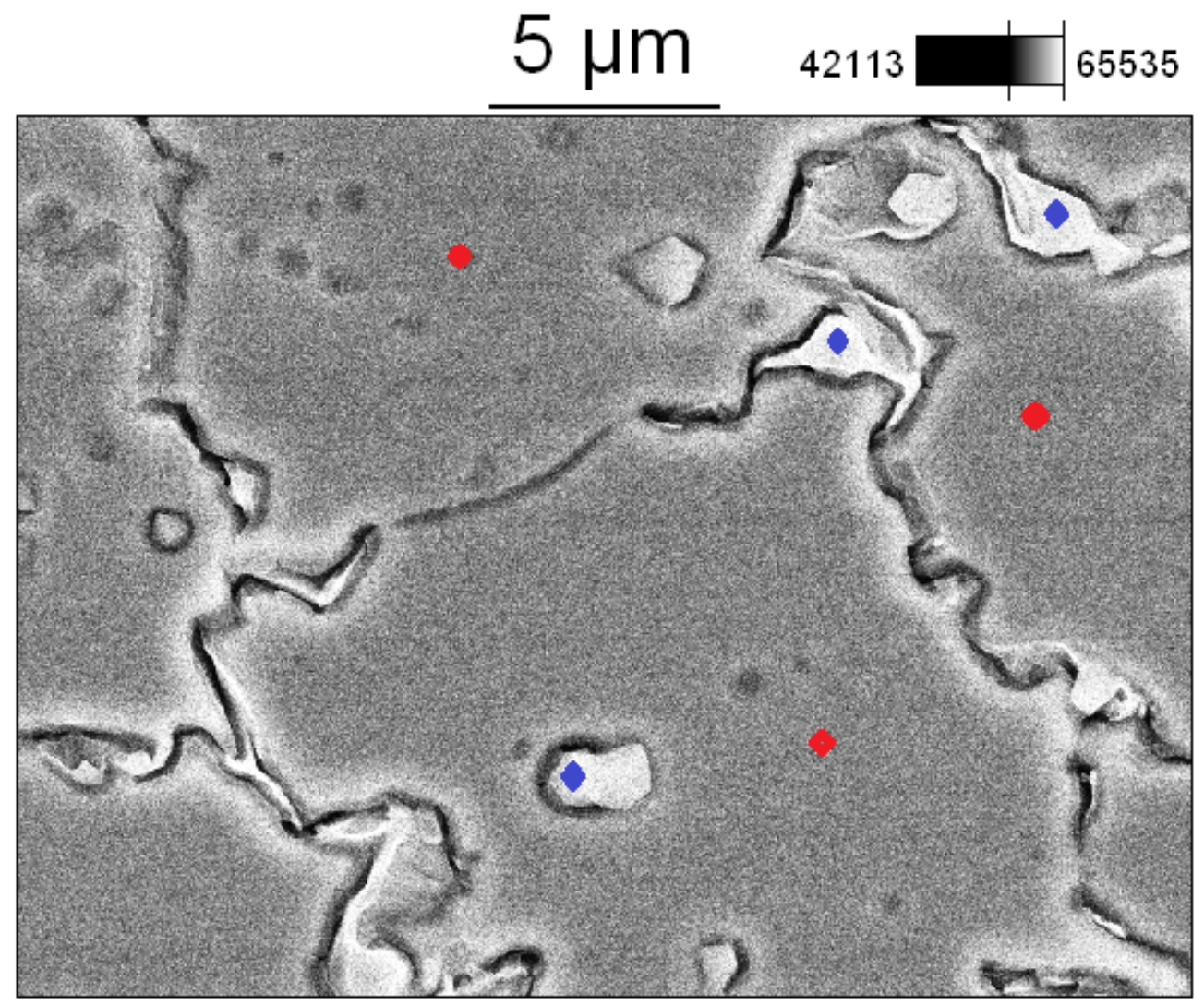

Figure 6.21: EDS Point scans of a high carbon wrought (course grains) of a CoCrMo alloy after corrosion 


\begin{tabular}{|c|c|c|c|}
\hline \multicolumn{4}{|c|}{ Weight Percentage } \\
\hline HC Wrought & Cobalt & Chromium & Molybdenum \\
\hline Red 1 & 59.55 & 29.05 & 11.40 \\
\hline Red 2 & 67.32 & 20.47 & 12.21 \\
\hline Red 3 & 65.42 & 22.45 & 12.13 \\
\hline Average & 64.10 & 23.99 & 11.91 \\
\hline St Dev & 4.05 & 4.49 & 0.45 \\
\hline Blue 1 & 29.39 & 63.94 & 6.68 \\
\hline Blue 2 & 29.17 & 64.34 & 6.48 \\
\hline Blue 3 & 29.36 & 64.36 & 6.28 \\
\hline Average & 29.31 & 64.21 & 6.48 \\
\hline St Dev & 0.12 & 0.24 & 0.20 \\
\hline
\end{tabular}

Table 6.4: EDS Chemical Analysis of Figure 6.21 


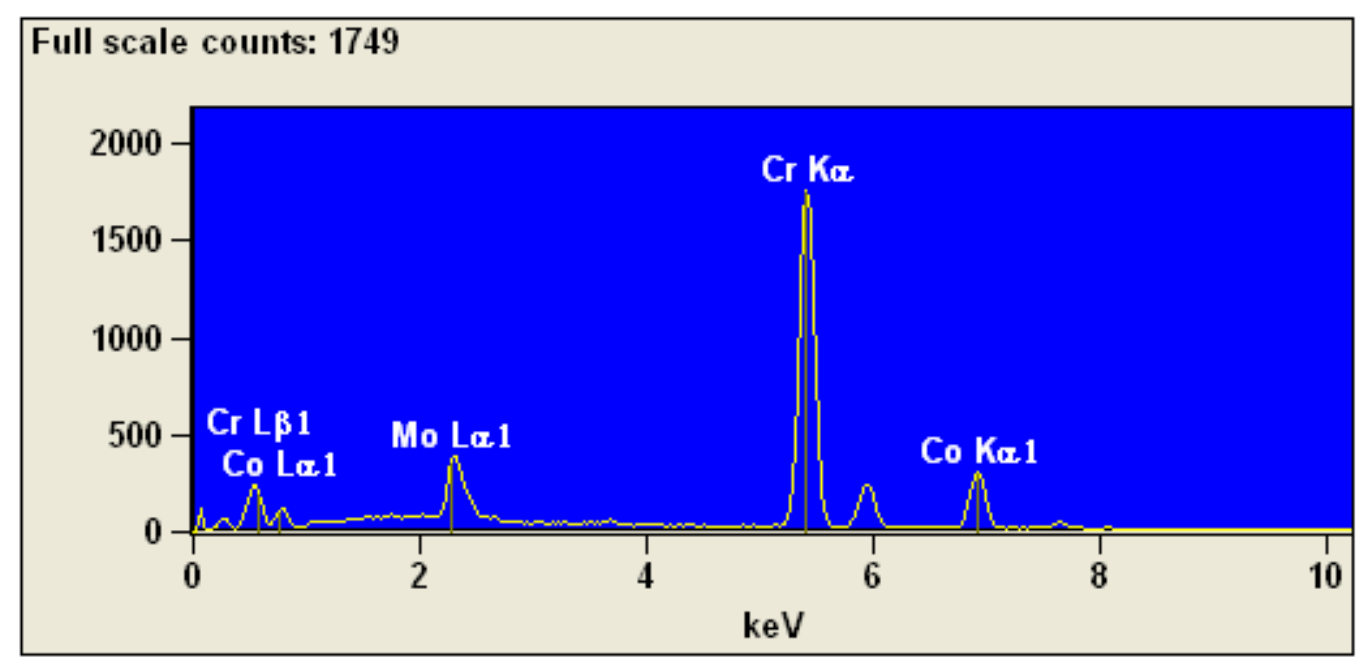

Figure 6.22: EDS Analysis of White Secondary Phase

Figure 6.22 represents the statistical chemical analysis of the white secondary phases found in figures 6.19-6.21 as represented by the blue dots in the image. It is clear that these regions are rich in chromium as evident by the average 65.46-weight percentage recorded in table 6.5. In the secondary phase, cobalt has an average weight percentage of 28.91. This is significantly less than the estimated 64.12 weight percentage found a polished sample. 


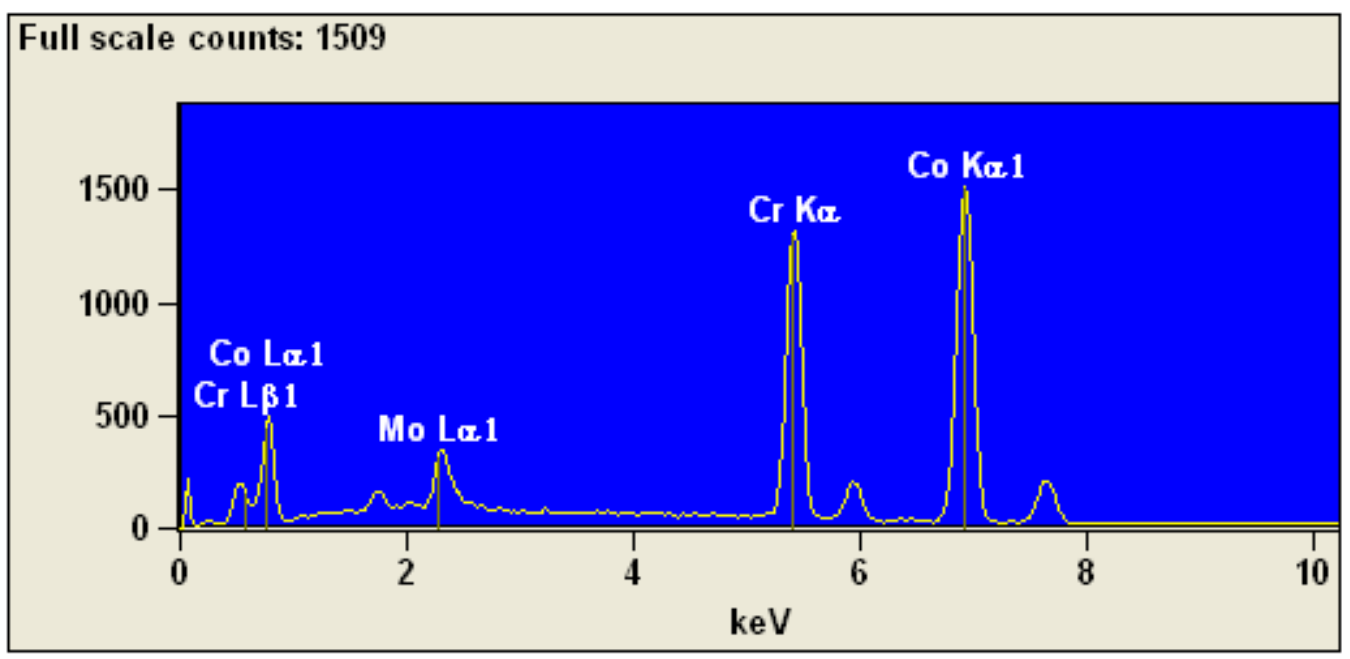

Figure 6.23: EDS Analysis of Grains

Figure 6.23 represents EDS analysis of the grains found in figures 6.19-6.21 and is represented by the red dots in these images. Just like in figure 6.18 the Co $\mathrm{K}_{\alpha 1}$ to be a higher peak than $\mathrm{Cr} \mathrm{K}_{\alpha}$ in figure 6.23. Both peaks are quite similar; however there is a slight difference in chemical composition of the polished sample and the grains as recorded in table 6.5. The most imminent difference is that molybdenum is found at more than twice the amount in the grains than in the polished sample.

\begin{tabular}{|c|c|c|c|}
\hline \multicolumn{4}{|c|}{ Weight Percentage } \\
\hline Sample & Cobalt & Chromium & Molybdenum \\
\hline Polished & 64.12 & 28.34 & 6.38 \\
\hline St Deviation & 3.50 & 2.72 & 1.38 \\
\hline Grains & 60.71 & 23.75 & 15.54 \\
\hline St Deviation & 10.75 & 5.30 & 5.94 \\
\hline Secondary Phase & 28.91 & 65.46 & 5.64 \\
\hline St Deviation & 0.36 & 1.54 & 1.24 \\
\hline
\end{tabular}

Table 6.5: Chemical Composition of Samples

Interestingly enough, the taken random EDS point analysis of samples results in an average chemical composition that has balanced cobalt - 28-weight percentage chromium - 6 weight percentage molybdenum. However the grains had higher amounts of 
molybdenum while the white secondary phases have significantly higher amounts of chromium and significantly lower amounts of cobalt. As the major alloying element, cobalt is found in the grains - as expected.

\subsection{Potentiodynamic Polarization Graphs}

From an engineering standpoint, the most important aspect of corrosion studies is to determine corrosion rates of the metal of interest. Corrosion systems are not equilibrium processes and thus thermodynamics cannot be applied. The anode is the electrode at which oxidation occurs, and the cathode is the electrode at which net reduction occurs. The exchange current density for any system must be determined experimentally. One such technique is potentiodynamic polarization whereby the potential of the system is incrementally scanned from cathodic to anodic potential. Alloys that contain iron, titanium, and chromium have an active-passive transition as detailed in figure 6.24.

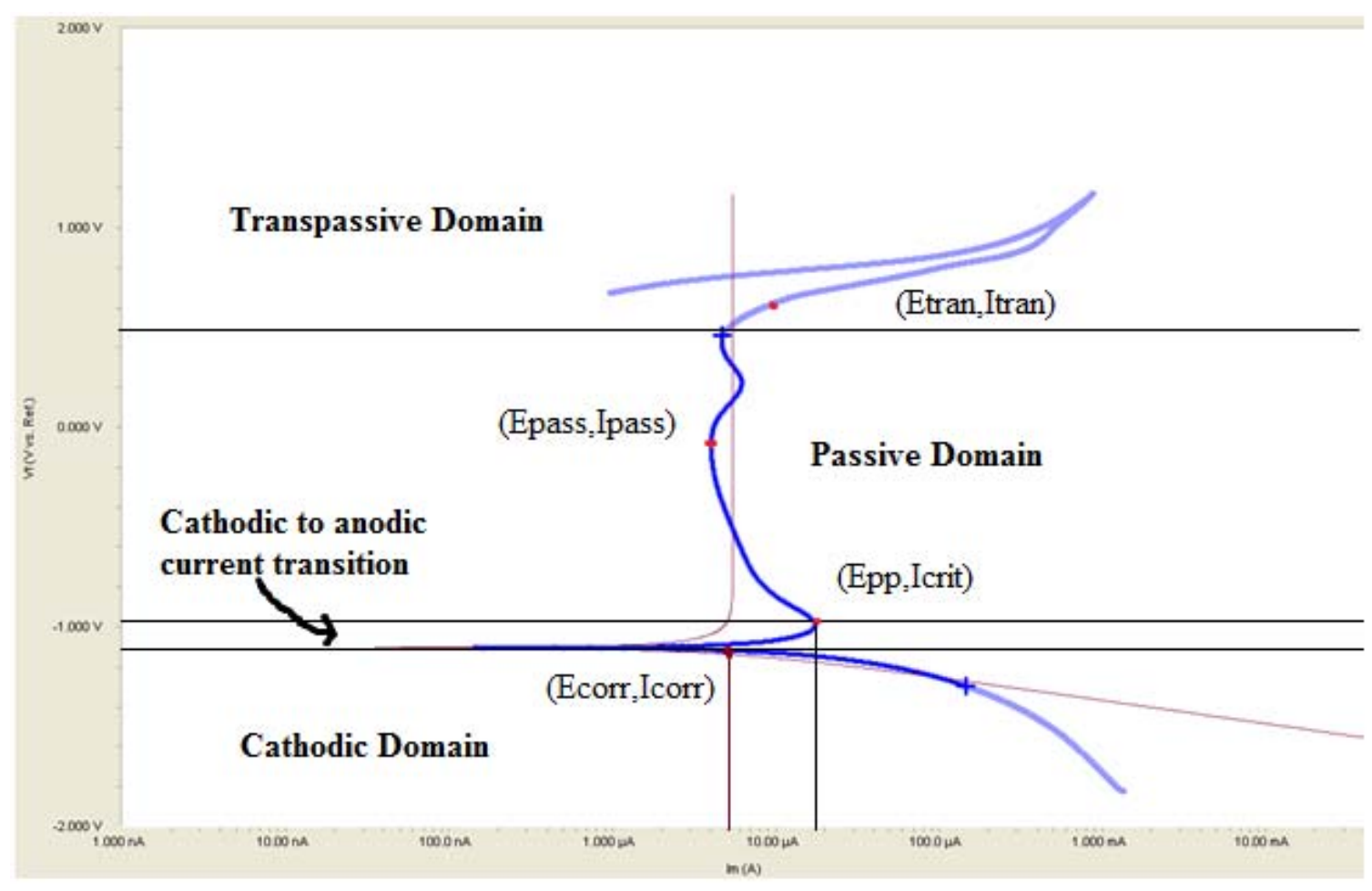

Figure 6.24: Typical potentiodynamic polarization graph for CoCrMo alloy 
At first these metals behave like non-passivating metals. As the potential is increased in the anodic direction, the metal exhibits a typical Tafel behavior. The dissolution rate rapidly increases in the cathodic domain. As the potential increases to a more noble value, passivity occurs. The dissolution rate drastically decreases and becomes independent of potential - known as the passive domain. Finally at very noble values, dissolution rate increases again in the transpassive domain. At $\mathrm{E}_{\text {corr }}$ the surface of the sample is undergoing oxidation and reduction at equal but opposite rates. Thereby, no net current is measured. Potentials negative of $\mathrm{E}_{\text {corr }}$ give rise to cathodic current while potentials positive of Ecorr give rise to anodic current. In transitioning towards passivity, exchange current density shifts from cathodic to anodic. An important aspect of activepassive metal transition is the position of the anodic current density maximum characterized by the primary passive potential $\left(\mathrm{E}_{\mathrm{pp}}\right)$ and the critical anodic current density for passivity ( $\left.\mathrm{I}_{\text {crit }}\right)$. The decrease is dissolution rate above $\mathrm{E}_{\mathrm{pp}}$ is a result of the passivation film growth. The ideal region for inducing passivity with the lowest exchange current density is at $-0.100 \mathrm{mVs}$ indicated by $E_{\text {pass }}$ and $i_{\text {pass. }}$ The beginning of the transpassive potential (Etrans) is marked with a transpassive exchange current density (itrans) at $10 \mu \mathrm{A} / \mathrm{cm}^{2}$. The rapid increase of the exchange current density in the transpassive domain is a result of the destruction of the passive surface oxide film at very positive potentials. Increases in temperature and hydrogen ion concentration tend to increase the critical anodic current density. It follows that an alloy tends to more readily passivate if it has a small critical anodic current density and an active primary passive potential. 


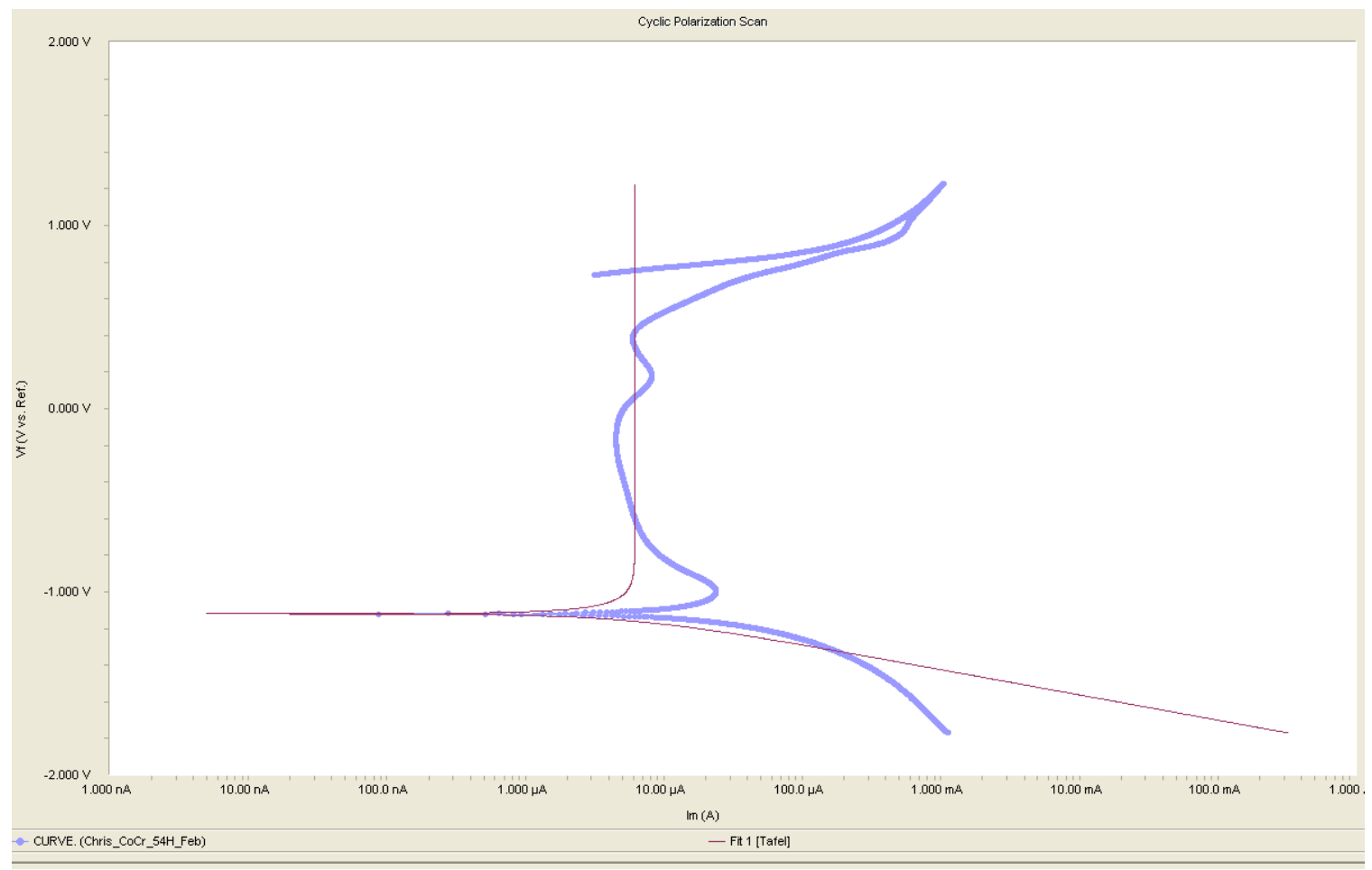

Figure 6.25: Potentiodynamic Polarization Curve for Low Carbon Wrought CoCrMo alloy

Figure 6.25 is the potentiodynamic polarization curve for low carbon wrought CoCrMo alloys. This graph is similar to the one found in Figure 6.24. It displays the cathodic domain, passive domain, and transpassive domain. Figure 6.25 is the corrosion response of the microstructure observed in images 6.1-6.4. For detailed potential and exchange current density for this type of microstructure refer to table 6.6 below. 


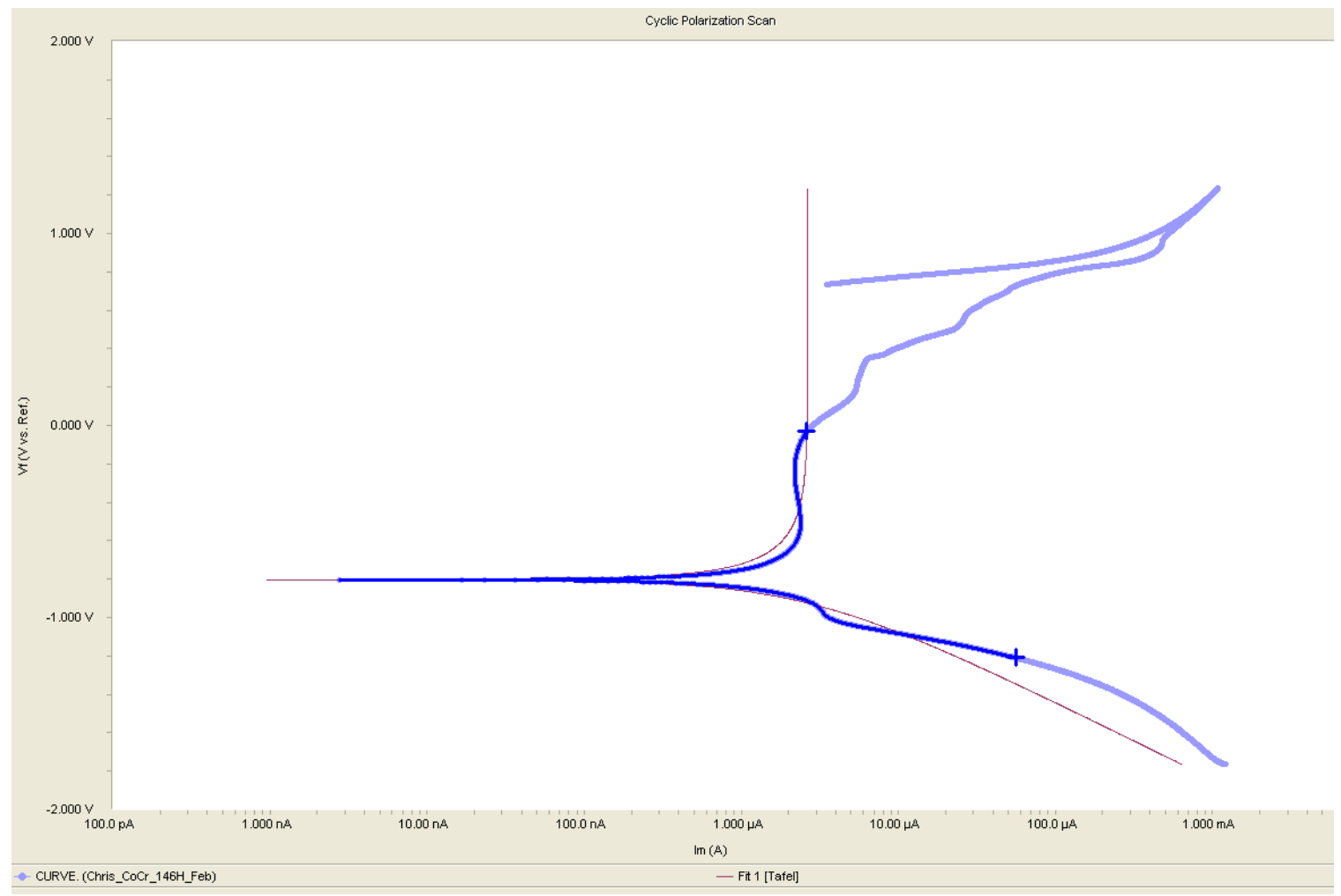

Figure 6.26: Potentiodynamic Polarization Curve for As-Cast CoCrMo Alloy

Figure 6.26 is the potentiodynamic polarization curve for as-cast CoCrMo alloys. It is slightly different from the one found in Figure 6.6. It displays the cathodic domain, passive domain, and transpassive domain; however, the passive domain is less pronounced and $\mathrm{E}_{\mathrm{pp}}$ and $\mathrm{i}_{\text {crit }}$ are difficult to distinguish. Figure 6.26 is the corrosion response of the microstructure observed in figures 6.5-6.8. Furthermore, figure 6.26 is representative of the chemical composition of the as-cast EDS found in figure 6.19 and chemical composition in table 6.5. For detailed potential and exchange current density for this type of microstructure refer to table 6.6 below. 


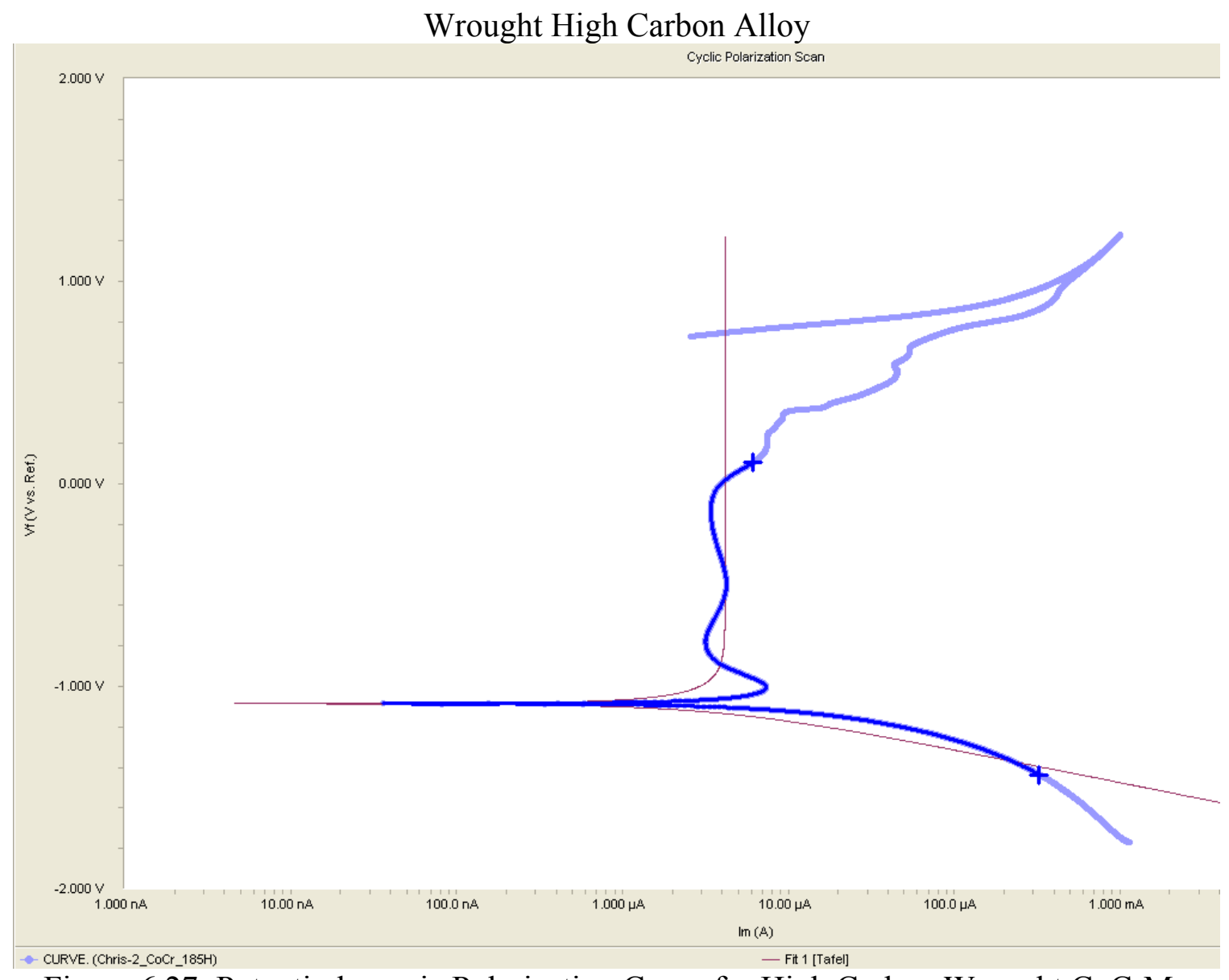

Figure 6.27: Potentiodynamic Polarization Curve for High Carbon Wrought CoCrMo Alloy

Figure 6.27 is the potentiodynamic polarization curve for high carbon wrought $\mathrm{CoCrMo}$ alloys. It is more similar to potentiodynamic polarization curve for the low carbon wrought $\mathrm{CoCrMo}$ alloy (figure 6.24) than the as-cast $\mathrm{CoCrMO}$ alloy (figure 6.25). It displays the cathodic domain, passive domain, and transpassive domain; however the transpassive domain is more pronounced and jagged. Figure 6.27 is the corrosion response of the microstructure observed in images 6.9-6.16. For detailed potential and exchange current density for this type of microstructure refer to table 6.6 below. 


\begin{tabular}{|c|c|c|c|c|c|c|}
\hline & As-Cast & st. deviation & LC Wrought & st. deviation & HC Wrought & st. deviation \\
\hline $\operatorname{Ecorr}(\mathrm{mV})$ & -693.00 & 96.60 & -1110.00 & 10.00 & -1086.67 & 5.77 \\
\hline $\operatorname{Icorr}\left(\mu \mathrm{A} / \mathrm{cm}^{\wedge} \wedge\right)$ & 4.95 & 0.37 & 5.80 & 0.36 & 4.23 & 0.81 \\
\hline $\operatorname{Epp}(\mathbf{m V})$ & N/A & $\mathrm{N} / \mathrm{A}$ & -994.10 & 5.90 & -996.13 & 22.49 \\
\hline $\operatorname{Icp}\left(\mu \mathrm{A} / \mathbf{c m}^{\wedge} 2\right)$ & N/A & N/A & 19.07 & 4.50 & 8.05 & 0.62 \\
\hline Epass (mV) & -100.60 & 0.00 & -101.53 & 1.62 & -100.60 & 0.00 \\
\hline Ipass $\left(\mu \mathrm{A} / \mathrm{cm}^{\wedge} 2\right)$ & 2.70 & 0.43 & 4.18 & 0.34 & 3.16 & 0.28 \\
\hline Epasspeak (mV) & 329.40 & 19.02 & 443.13 & 35.33 & 254.43 & 38.84 \\
\hline Ipasspeak $\left(\mu \mathrm{A} / \mathrm{cm}^{\wedge}{ }^{\wedge}\right)$ & 6.17 & 0.05 & 5.44 & 0.55 & 7.79 & 0.56 \\
\hline Etran1 (mV) & 394.50 & 19.02 & 577.93 & 61.58 & 337.27 & 21.31 \\
\hline $\operatorname{Itran} 1\left(\mu \mathrm{A} / \mathrm{cm}^{\wedge}{ }^{\wedge}\right)$ & 10.02 & 0.04 & 10.05 & 0.02 & 10.03 & 0.08 \\
\hline Etran2 (mV) & 783.03 & 17.09 & 792.97 & 0.12 & 769.23 & 27.09 \\
\hline $\operatorname{Itran} 2\left(\mu \mathrm{A} / \mathrm{cm}^{\wedge}{ }^{\wedge}\right)$ & 99.99 & 0.35 & 100.06 & 0.24 & 99.72 & 0.12 \\
\hline deltaE (mV) & 1084.50 & N/A & 1572.03 & N/A & 1333.40 & N/A \\
\hline
\end{tabular}

Table 6.6: in-Vitro Corrosion data of three different CoCrMo alloys

Table 6.6 is the in-Vitro corrosion data of three types of CoCrMo alloys: As-cast, low carbon (LC) wrought, and high carbon (HC) wrought. The as-cast alloy is represented by the ASTM F75 alloy, while the LC and HC wrought alloys are two subsets of the ASTM F1537 with different concentrations of carbon within the alloy. Three in-Vitro corrosion potentiodynamic polarization tests were conducted for each alloy. Table 6.6 provides the averages and standard deviations for each metric. The electrode potential is measured in millivolts $(\mathrm{mV})$ in reference to the silver/silver chloride $(\mathrm{Ag} / \mathrm{AgCl})$ electrode, while the exchange current density is measured in microampere per centimeter squared $\left(\mu \mathrm{A} / \mathrm{cm}^{2}\right)$. The exposed surface area for each sample was standardized with by using a rubber O-ring with an inner cross sectional area of $0.5901 \mathrm{~cm}^{2}$. The samples have a density of $8.5 \mathrm{~g} / \mathrm{cm}^{3}$ and equivalent weight of 26.069. The most noticeable difference, figure 6.28 , is that the as-cast group has higher $\mathrm{E}_{\text {corr }}$ values relative to LC and HC wrought; however, all allow groups have similar $\mathrm{i}_{\text {corr }}$ values. In order from lowest to highest $\mathrm{i}_{\text {corr }}$ values: $\mathrm{HC}$ wrought $<$ as-cast $<\mathrm{HC}$ wrought. Furthermore, LC Wrought has a critical passivation current density twice as high as $\mathrm{HC}$ wrought. To better compare the potentiodynamic 
polarization data several additional points were measured. The passivation current was measured at $-100 \mathrm{mV}(\mathrm{Ag} / \mathrm{AgCl})$. Furthermore the apex of the passivation domain was measured along with two points in the transpassive region. Etrans1 represents the potential at $10 \mu \mathrm{A} / \mathrm{cm}^{2}$, while Etrans 2 represents the potential at $100 \mu \mathrm{A} / \mathrm{cm}^{2}$. This provides the change in potential in the transpassive domain with an increase in one order of magnitude of exchange current density. DeltaE is the span of the passive domain calculated by $E_{\text {passpeak }}-E_{p p}$. This is an important measurement because it provides and estimation as to how width of the passivation domain for each alloy. The size of the passivation domain is in order from largest to smallest: LC Wrought > HC Wrought > As-Cast. 


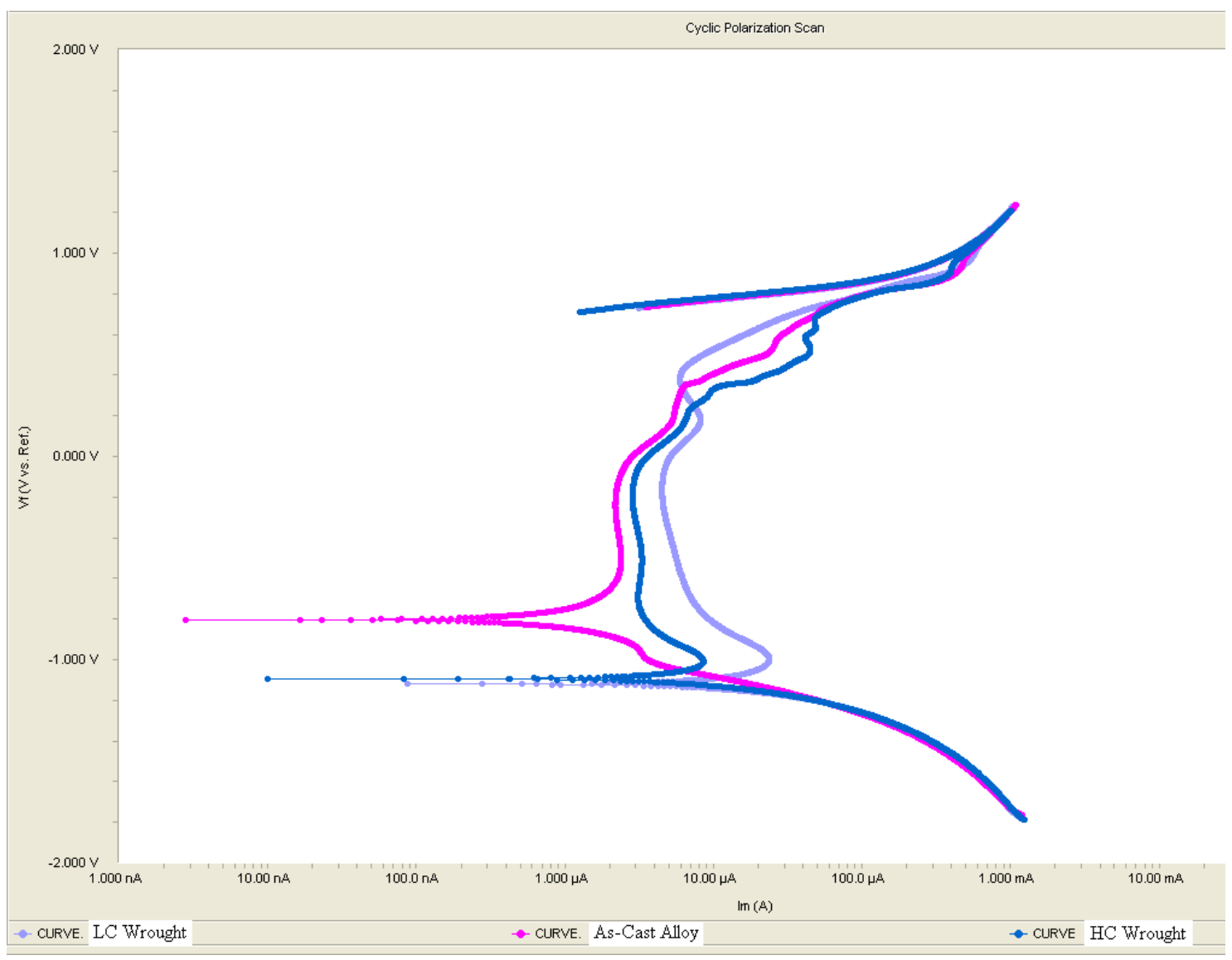

Figure 6.28 Overlay of the electrochemical behavior of the low carbon wrought, as-cast, and high carbon wrought alloys

\section{Chapter 7 Discussion}

\subsection{Processing and Microstructure}

As-cast CoCrMo alloys registered as ASTM F75 have been utilized for their ease of manufacturability. Casting conditions such as melt temperature, mold preheat, and cooling rate can influence the microstructure of the alloy and ultimately the material properties of the cast material. Chvorinov's rule states that total solidification time ( $\left.\mathrm{t}_{\mathrm{f}}\right)$ is related to volume $(\mathrm{V})$ and surface area $(\mathrm{A})$ of the casting

$t_{f}=C\left(\frac{V}{A}\right)^{2}$ 
Where C is Chvorinov's number, a constant specific to each material. Kaiser et al., reported that as secondary dendrite arm spacing (SDAS) increased the section thickness increased, and can be visualized in figure 7.1. Additionally a higher surface area to volume ratio results in a finer microstructure.

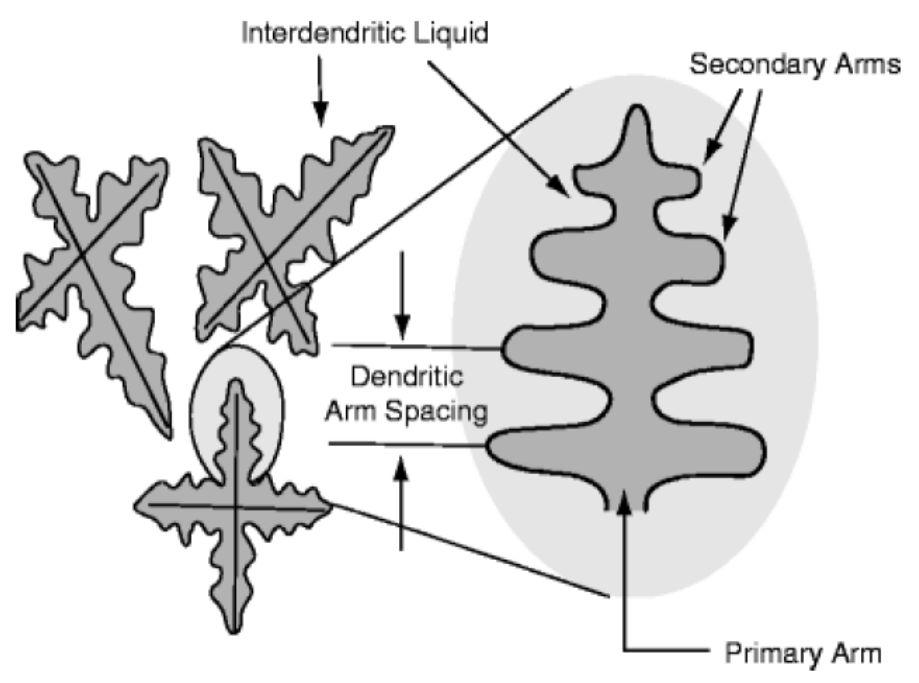

Figure 7.1: As-cast dendrite structure

A higher SDAS is a result from slower cooling rates or increased solidification time. Microhardness is inversely related to SDAS. Larger SDAS results in lower hardness values [101]. Thus the microstructure and microhardness fundamentally important parameters to better understand the processing history of a metallurgical sample. For example, the as-cast alloy has a dendrite matrix with interdendritic and grain boundary precipitation. Carbides are a common precipitate that improves the microhardness of this alloy, and ultimately its wear resistance. Processing parameters influence the structure and frequency of carbides in the microstructure. 


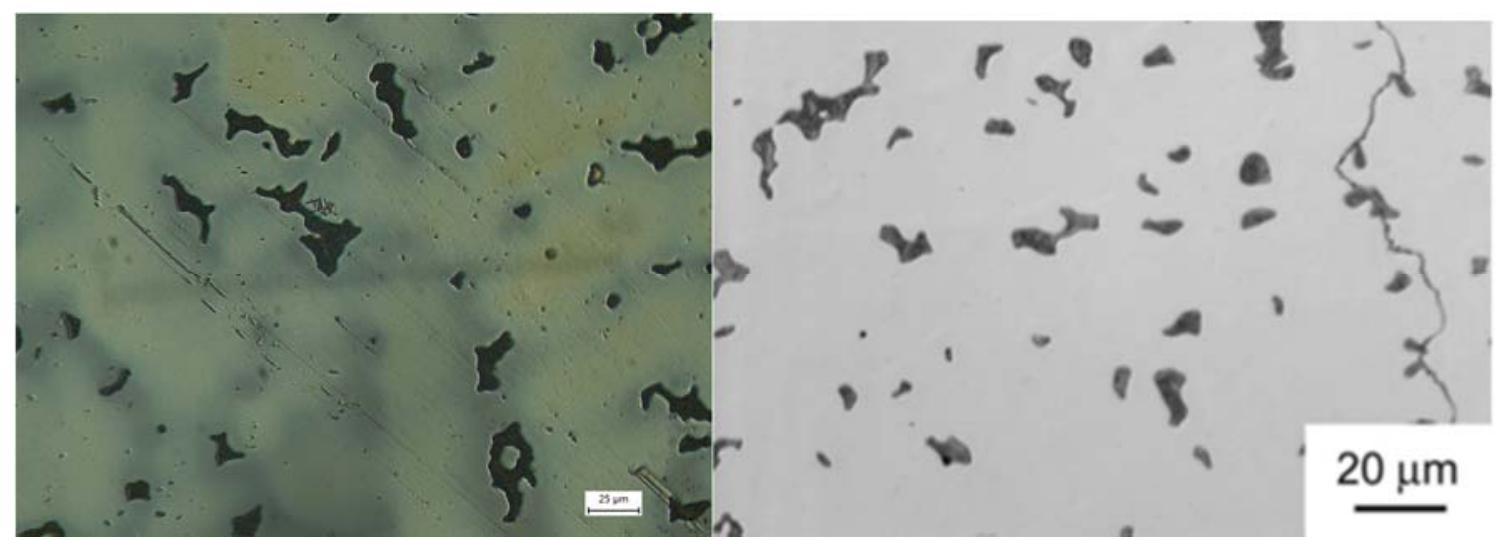

Figure 7.2: As-Cast CoCrMo microstructure unknown processing parameters (left)

Figure 7.3: As-Cast CoCrMo microstructure with $0.35 \mathrm{wt} \%$ carbon (right) [102]

Mineta et al., investigated the carbide formation and dissolution of the as-cast CoCrMo alloy with solution treatment at temperatures ranging from 1473 to $1548 \mathrm{~K}$ and hold times between 0 to 43.2 kiloseconds (ks). They found that increasing carbon content from 0.12 wt $\%$ to $0.35 \mathrm{wt} \%$ increased the precipitation frequency. Temperature up to $1548 \mathrm{~K}$ was required to completely dissolve all precipitates in the alloy with $0.35 \mathrm{wt} \%$ carbon. Increasing the hold time also resulted in decrease precipitate formation [102]. Carbide precipitation on the microstructure of CoCrMo alloy is a direct result of heat-treatment conditions, temperature and time. Figure 7.2 is a micrograph of the as-cast sample at 200x magnification with a $25 \mu \mathrm{m}$ measure bar. Based on microstructure comparison figure 1 has similar features to figure 2. Figure 7.3 is microstructure of an as-cast CoCrMo sample with $0.35 \mathrm{wt} \%$ carbon reproduced from Mineta et al. The microstructure in figure 7.3 is a result of processing at $1473 \mathrm{~K}$ with a hold time of $43.2 \mathrm{ks}$. The similarity in microstructure from the retrieved sample (figure 7.2) and the literature (figure 7.3) suggests that the retrieve as-cast sample may have been processed at a lower temperature $(<1500 \mathrm{k})$. The duration of this treatment can only be hypothesized; however it is clear the 
carbides precipitation is abundant. Therefore, it is also possible that this sample can be classified with carbon content near $0.35 \%$ weight. To completely dissolve carbide precipitates it is necessary for as-cast samples to undergo high heat-treatment temperature $(>1548 \mathrm{~K})$ and long holding times ( $>43.2 \mathrm{ks})$. Montero-Ocampo et al., reports that as-cast fabrication of CoCrMo alloys generates large defects in the microstructure. These defects include large grains, porosity, interdendritic carbides, and solute segregation. These defects result in a more brittle material with poor strength. Additionally the report that the interdendritic is a eutectic with a mixture of $\mathrm{M}_{23} \mathrm{C}_{6}$ carbides, $\sigma$ phase, and FCC rich cobalt face [71]. Kaiser et al., reports that the microstructure of investment cast specimens are dendritic matrix with secondary phases precipitated at grain boundaries and interdendritic zones. The group provided additional support with EDS analysis that the main matrix of the as-cast sample is high in cobalt while the precipitates indicate high concentration of chromium and molybdenum. This further supports the general acceptance that the main secondary phase is the $\mathrm{M}_{23} \mathrm{C}_{6}$ carbides composed of Chromium and Molybdenum. They also reported microhardness measurements between 310-350HV for the matrix, and 400-600HV for the carbides. The carbides clearly increase the overall hardness of the alloy [103]. Figure 7.4 shows the microstructure reported from Kaiser at a low and high magnification. This is in comparison to the retrieval sample in figure 7.5, showing a low (50x) and high (2000x) magnification image of the microstructure. An alternative method in determining cooling rates for dendritic microstructures is calculation of dendrite arm spacing. Secondary dendrite arm spacing is refined with increasing cooling rates by the equation 
$\lambda_{2}=5.5\left(M t_{f}\right)^{1 / 3}$

where $\mathrm{M}$ is a constant and to is the total solidification time.

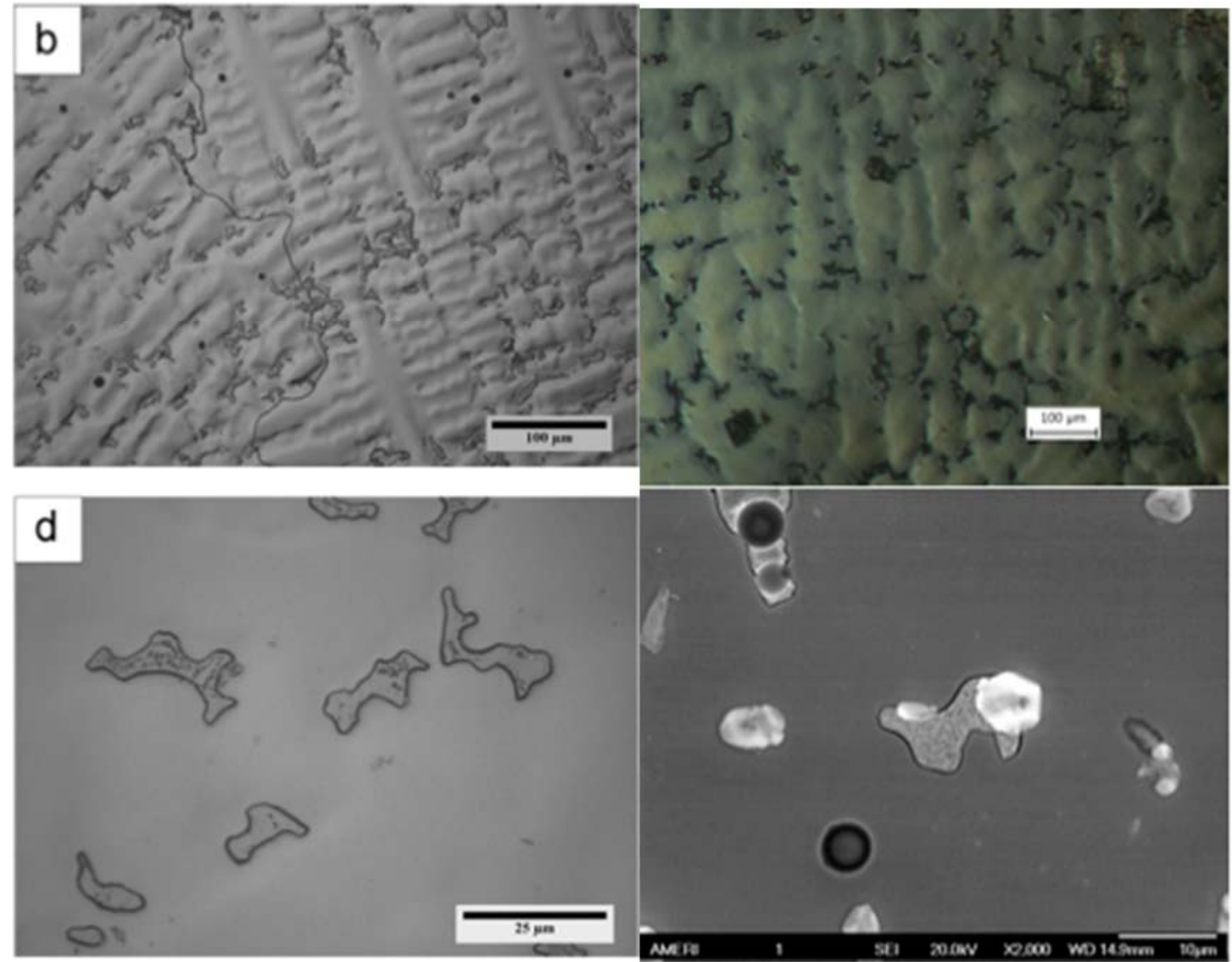

Figure 7.4: Typical as-cast microstructure low magnification and higher magnification (left)

Figure 7.5: As-cast microstructure low magnification and higher magnification (right) Wrought production can create CoCrMo alloy with better mechanical properties. Wrought production begins with vacuum induction melting of the primary alloy elements followed by electroslag improve ingot cleanliness and structure. The ingots are homogenized at $1250^{\circ} \mathrm{C}$ for 5 hours. After ingots are homogenized they are forged into a reroll billed and rolled to a final size. Controlled war/hot working or annealing can 
determine the final microstructure and mechanical properties. Forging creates alloy deformation, increasing the stored strain energy. Additionally forging can reduce the average grain size of the alloy. The Hall-Petch relates yield stress to grain size

$\sigma_{y}=\sigma_{0}+\frac{k_{y}}{\sqrt{d}}$

Whereby

$\sigma_{\mathrm{y}}$ is the yield stress

$\sigma_{0}$ is the lattice yield stress to dislocation motion (material specific)

$\mathrm{k}_{\mathrm{y}}$ is the strengthening coefficient (material specific)

$\mathrm{d}$ is the average grain diameter

Carbon content in the form of carbides can further increase the strength and hardness of this alloy. Turrubiates-Estrada et al., investigated the FCC $\rightarrow \mathrm{HCP}$ martensitic transformation in a $\mathrm{Co}-27 \mathrm{Cr}-5 \mathrm{Mo}-0.23 \mathrm{C}$ alloy processed by powder metallurgy. The investigators received a powder metallurgy sample that was processed through the ASTM F799 with a grain size of $3.15 \mu \mathrm{m}$. The microstructure from the as-received sample if found in figure 7.6. EDS analysis of the dark particle represented by the top area and the light grey/white secondary phase is labeled by the bottom arrow. 


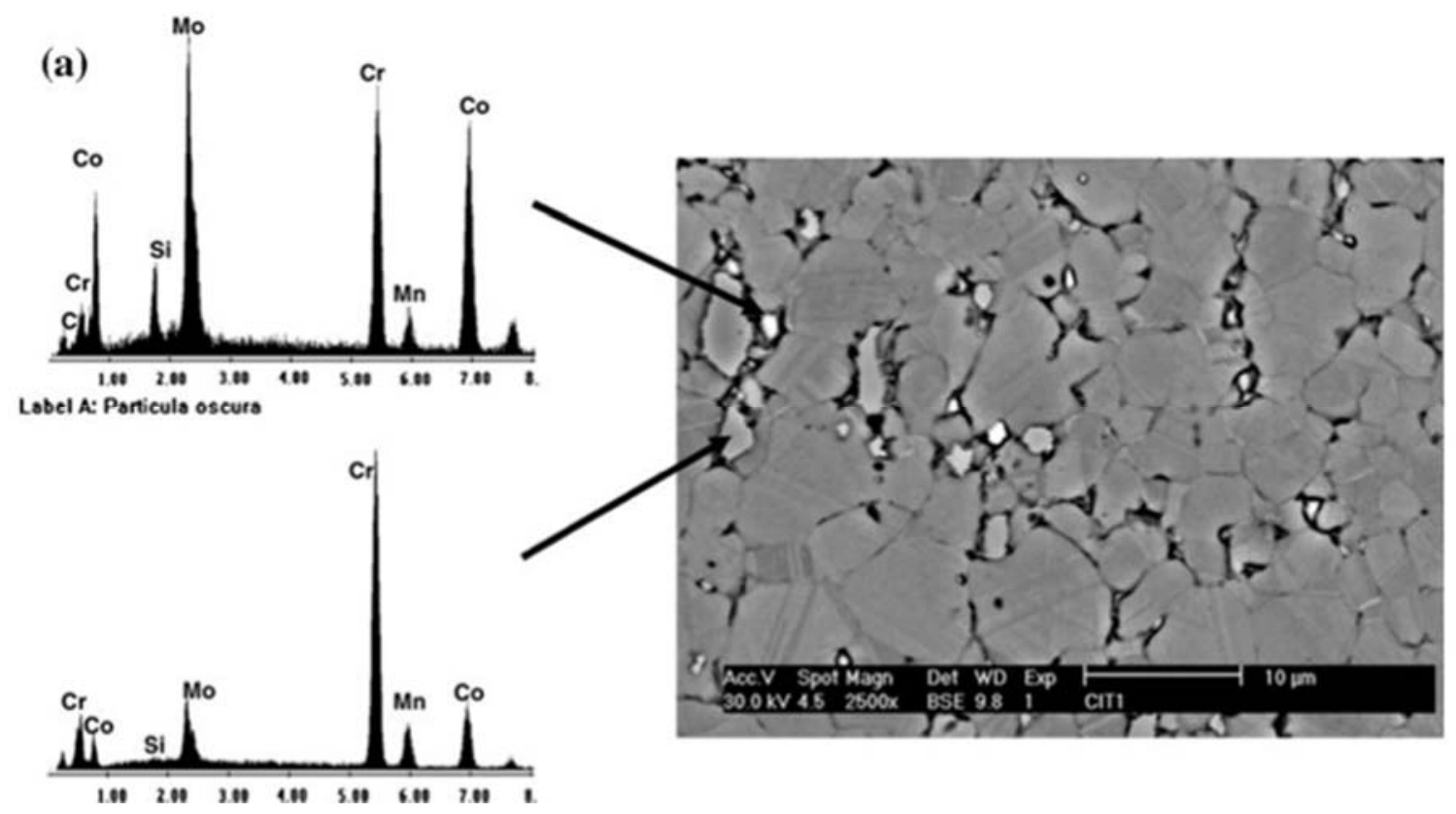

Figure 7.6: EDS analysis of as-received microstructure: Powder Metallurgy + Forging

Figure 7.6 shows the microstructural features of the co-based alloy in the as-received condition. The alloy was processed by powder metallurgy and exhibits some residual intrinsic porosity. Notice that the microstructure consists of a matrix of internally twinned equiaxed grains with second phases predominantly along the grain boundaries. An evaluation of the chemical composition of the second phases present using energy dispersive X-ray spectroscopy in the SEM indicated that these phases were probably alloyed carbides as they were $\mathrm{Cr}$ and Mo rich and they contained some carbon. [104] The HCP phase is formed from metastable FCC through a martensitic reaction whose nucleation stage is thermally activated at temperatures above 785 . Maximum isothermal transformation rates were found to occur at $825-850$. Figure 7.7 is the as-received hip implant microstructure with its EDS spectrum. The similarity in microstructure between figure 7.6 and figure 7.7 suggests that this sample possibly was formed through powder metallurgy and then the sample was forged. 


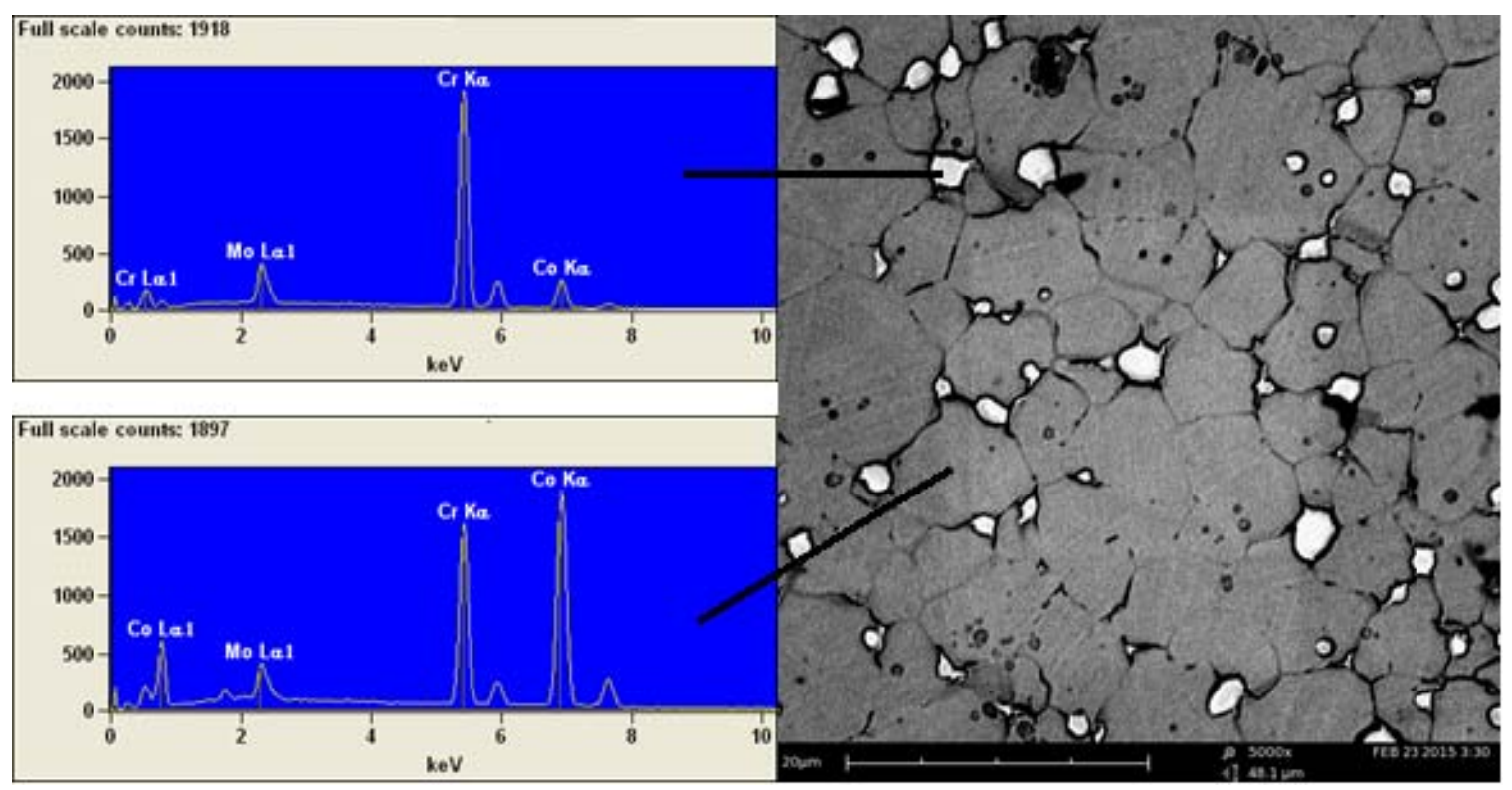

Figure 7.7: EDS analysis of as-received microstructure from hip prosthesis

From the EDS interpretations of Figure 7.6 and Figure 7.6 there are regions of the microstructure that are exclusively rich in chromium (small cobalt peak around $7 \mathrm{KeV}$ ).

\subsection{Electrochemical Behavior}

Milosev et al. studied the passive oxide film of CoCrMo alloys in various physiological solution reporting that the CoCrMo passive range extends from $-300 \mathrm{mV}$ to $+500 \mathrm{mV}$ versus saturated calomel electrode (SCE). A silver/silver chloride electrode is $-42 \mathrm{mV}$ versus SCE. The transpassive domain begins above $+500 \mathrm{mV}$. It was found that the oxide layer is composed mainly of chromium (III) oxide/hydroxide and is up to $2 \mathrm{~nm}$ thick. Calcium phosphate was found to precipitate on the surface if the electrolyte contained calcium and phosphate ions, such as Hanks solution. Phosphate ions tend to shift the transpassive domain to more negative values and induces the release of $\mathrm{Cr}(\mathrm{IV})$ to produce 
chromium phosphate precipitates - $\mathrm{CrPO}_{4}$. Ultimately, solution chemistry affects both the anodic and cathodic parts of the polarization curve [105]. Vidal et al., studied the polarization behavior of CoCrMo alloy in different solutions. The polarization curve can be divided into four regions: 1) cathodic domain, 2) transition from cathodic to anodic current, 3) passive plateau, and 4) transpassive domain. The cathodic domain includes potentials below $-1.1 \mathrm{~V}_{(\mathrm{Ag} / \mathrm{AgCl})}[67]$. This transition is visualized in both figure 7.8 and figure 7.9 below and discussed in the results section. One limitation of in-Vitro testing is that it does not fully replicate the in-Vivo behavior at the taper junction. Simulated physiological solutions lack biomolecules. Studies have incorporated albumin into solution and have found that is acts as a competitive inhibitor, while phosphate acts an anodic inhibitor $[67,105]$. 

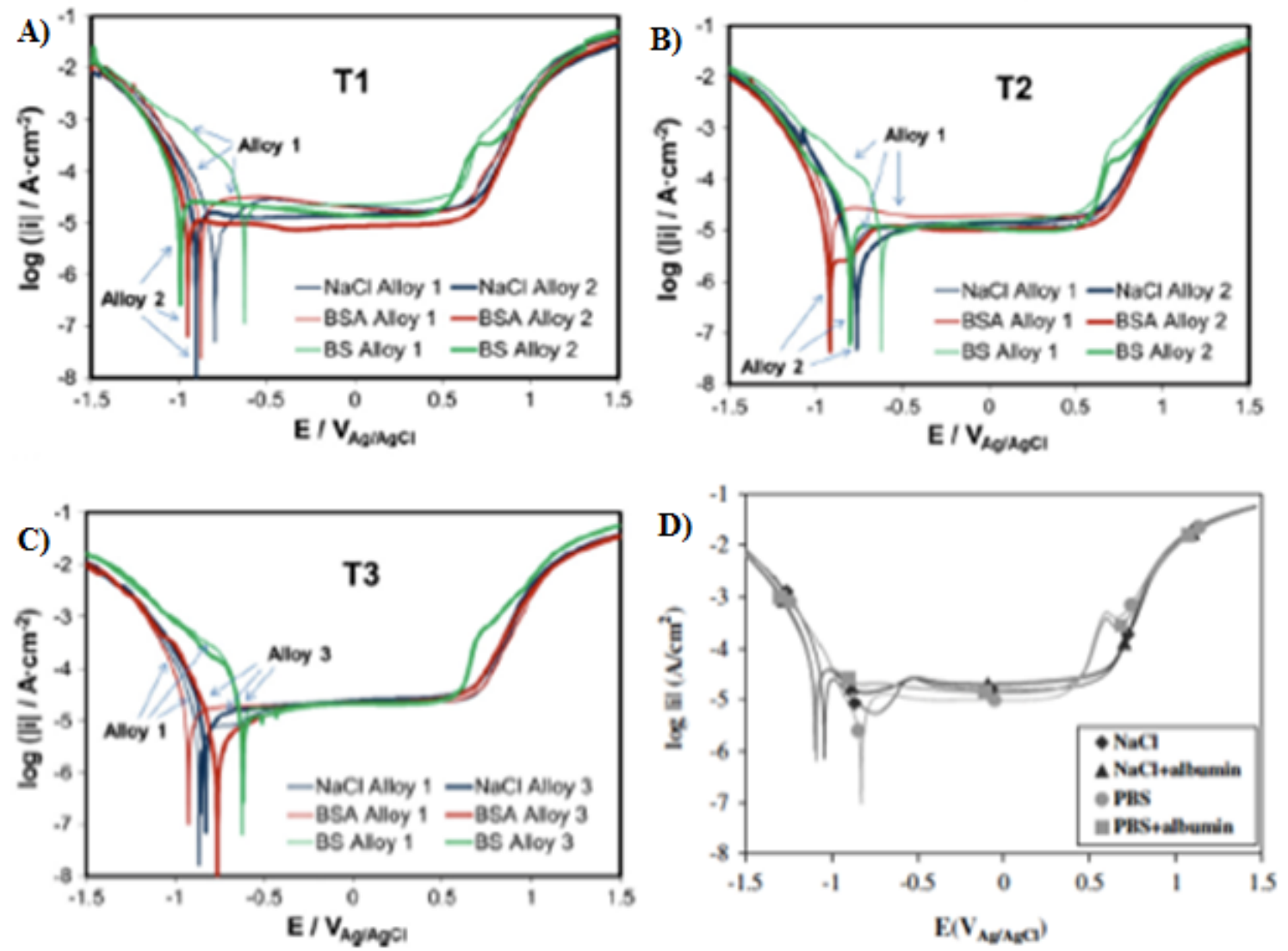

Figure 7.8: CoCrMo electrochemical behavior Images A-C were reproduced from (Vidal 2013 [106]) and image D was reproduced from (Vidal 2008 [67])

Vidal et al., (2008 and Figure 7.8D) investigated the chemical behavior of the CoCrMo alloy Protasul-20 (chemical composition in accordance to ISO standard 5832-12) ion four electrolytic solutions. From figure 7.8D it is clear that solution chemistry effects the electrochemical behavior of the CoCrMo alloy especially the corrosion potential $\mathrm{E}_{\mathrm{corr}}$, corrosion current density $\mathrm{i}_{\text {corr, }}$ and transpassive dissolution $\mathrm{E}_{\text {tran. }}$ The group reported that albumin increased the corrosion resistance of this alloy. Additionally CoCrMo forms a thicker and more protective film than AISI 316L stainless steel. Electrochemical impedance spectroscopy was utilized to study the surface film oxide thickness. This 
technique was not utilized in this thesis; however, it may be beneficial as part of future investigation. Vidal et al., (2013) investigated the electrochemical behavior of as-Cast CoCrMo alloy with different weight percentage of carbon content. Potential dynamic curves from this group are reproduced in figure 7.8A-C. They report that both the amount of carbides in CoCrMo alloys and the chemical composition of the simulated body fluid influence the electrochemical properties of the CoCrMo alloy. Lower carbide content (less weight percentage carbon) results in a more homogeneous microstructure and thus allows chromium to more readily form the passive oxide film. Passivation is controlled by microstructure and carbide content. The presence of carbides reduces the available of chromium to form a passive surface layer. Electrochemical data from both groups along with the data reported in the results section in this dissertation is reproduced in table 7.1 below. 


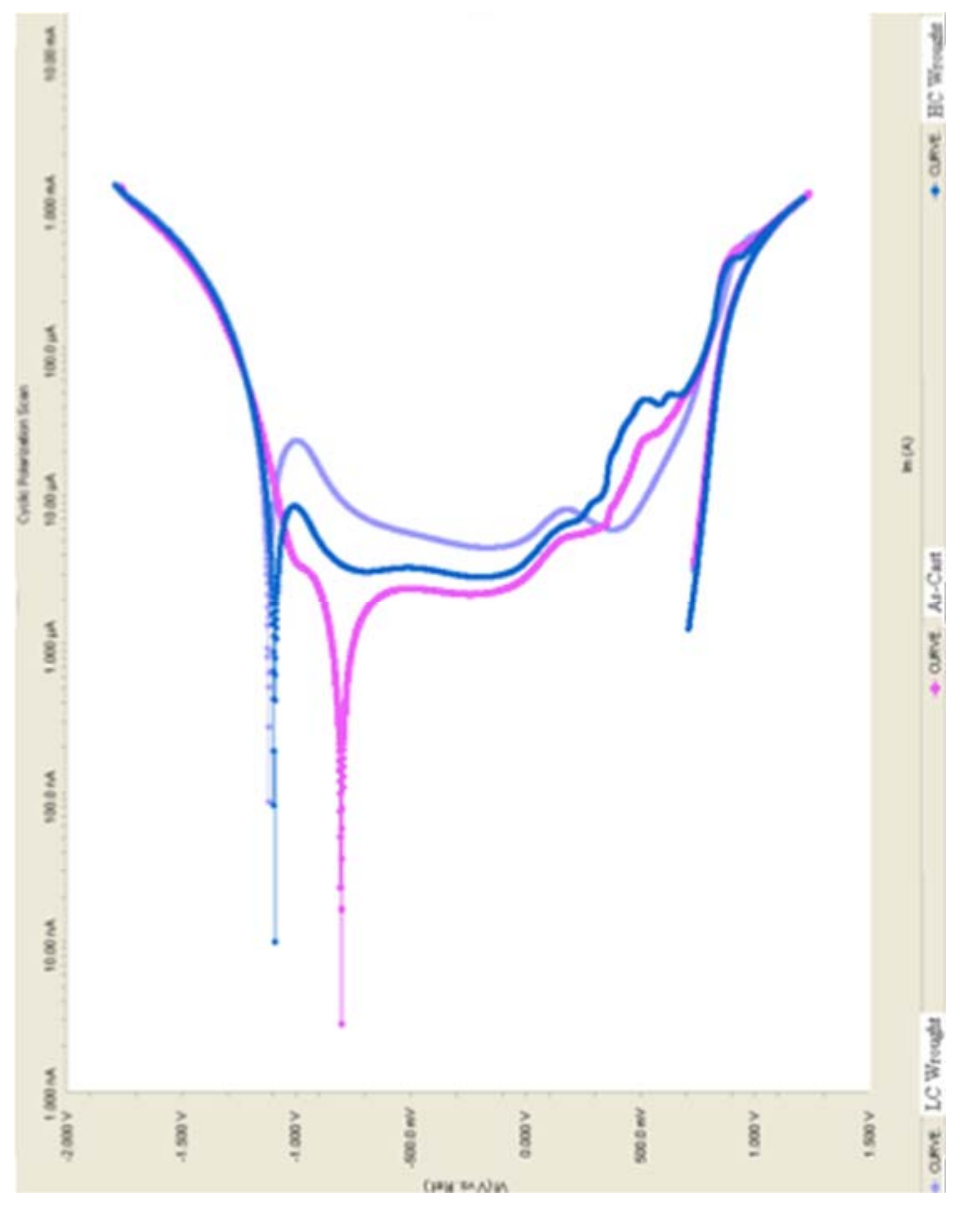

Figure 7.9: Inverted Overlay of potentiodynamic graphs from result section

Figure 7.9 is an inverted overlay of the potentiodynamic graphs of the low carbon (LC) wrought CoCrMo alloy (light blue curve), as-cast CoCrMo alloy (pink curve), and high carbon (HC) wrought CoCrMo alloy (dark blue curve) from the results section in Hanks solution. The inverted overlay of the data in figure 7.9 was generated to allow easier comparison to the potentiodynamic polarization graphs in figure 7.8. The most noticeable difference in electrochemical behavior (figure 7.9) is the corrosion potential between the as-cast microstructure and the wrought microstructure. Corrosion current density and passivation current density for all three microstructures are similar. However, the transpassive behavior of all three microstructures varies between 10 to $100 \mu \mathrm{A} / \mathrm{cm}^{2}$. 


\begin{tabular}{lllcccc} 
Source & Microstructure & Solution & Ecorr $(\mathbf{m V})$ & Icorr $(\boldsymbol{\mu A} / \mathbf{c m} \wedge \mathbf{2})$ & Ipass $(\boldsymbol{\mu A} / \mathbf{c m} \wedge \mathbf{2})$ & $\mathbf{E t r a n 1}(\mathbf{m V})$ \\
Thesis Results & As-Cast & Hanks & -693 & 4.95 & 4.18 & 553.27 \\
& LC Wrought & Hanks & -1110 & 5.80 & 2.70 & 439.87 \\
\multirow{5}{*}{ Vidal (2008) } & HC Wrought & Hanks & -1087 & 4.23 & 3.16 & 337.27 \\
& ISO 5832-12 & NaCl & -1090 & 9.60 & N/A & N/A \\
& ISO 5832-13 & NaCl+Alb & -1140 & 11.50 & N/A & N/A \\
& ISO 5832-14 & PBS & -880 & 2.10 & N/A & N/A \\
Vidal 2013 & ISO 5832-15 & PBS+Alb & -1140 & 11.60 & N/A & N/A \\
& Various & NaCl & -805 & 3.10 & 21.13 & 708 \\
& Various & NaCl+Alb & -854 & 6.49 & 20.75 & 637 \\
& Various & Bovine Serum & -721 & 10.02 & 21.33 & 543
\end{tabular}

Table 7.1: Summary of in-Vitro electrochemical behavior of CoCrMo alloys

Table 7.1 summarizes the electrochemical behavior of three groups. A key take away is that both microstructure and electrolyte solution chemistry influence the electrochemical behavior of CoCrMo alloys. Both Vidal 2008 and Vidal 2013 report different Ecorr and Icorr values. The authors were on both publications this highlights the difficulties of experiments to analysis the electrochemical behavior of this alloy. Hanks solution was utilized because it contained other ions such as magnesium, calcium, and bicarbonate that may not be included in PBS. In general, electrochemical behavior is within the bounds of that reported in the literature. The size of the passivation domain is in order from largest to smallest: LC Wrought $>$ HC Wrought $>$ As-Cast. Thereby, this study suggests that LC Wrought CoCrMO alloys have the best corrosion resistance properties. Similarly, prior literature has reported that $\mathrm{HC}$ Wrought CoCrMo alloys have lower corrosion resistance because carbides prevent chromium oxide passivation. This can lead to various pitting and intergranular corrosion around surface carbides. It has already been well established that as-cast alloys have inferior corrosion resistance properties compared to wrought alloys. The results in this thesis agrees with the literature. 


\section{Chapter 8: Conclusion}

Orthopedic manufactures may be changing the post processing mechanisms of orthopedic alloys such as CoCrMo. One of the limitations of retrieval studies is that implants under analysis were manufactured a decade ago. Manufactures may have altered their production processes. This makes it challenging when comparing past manufacturing techniques to newest generation of when comparing material properties. Furthermore there is a shift to use ceramic $\left(\mathrm{Al}_{2} \mathrm{O}_{3}\right)$ instead of metal $(\mathrm{CoCrMo})$ as the material of choice for the femoral head component of the alloy. It has been established that microstructure influences the both the electrochemistry and tribology properties of CoCrMo. Carbides are utilized to improve the overall hardness of the alloy and thus improve its wear resistance. This is an important material property for the femoral head component; however carbides may also deplete chromium in the metal matrix The lack of chromium to form a surface oxide reduces the alloy's corrosion resistance. Solution chemistry and surface finish will affect in-vitro corrosion results. By measuring the passivation span of the cyclic polarization curve, the results suggest that LC Wrought CoCrMo alloys have the best corrosion resistance properties. This is agreement with current literature theory. Although CoCrMo has been readily available as an orthopedic alloy since the 1930 s, continued research is needed to enhance the material properties. Future studies will include investigating in-vitro corrosion generated from mating different CoCrMo microstructures and dissimilar alloys (Ti-6Al-4V with Co-28Cr-6Mo). Hardness measurements will need to be acquired of all samples to be able to then perform tribology experiments. Wear is an equality important property to measure materials used in weight bearing prostheses. 


\section{References}

1. Chang, C.Y. and A.J. Huang, MR imaging of normal hip anatomy. Magn Reson Imaging Clin N Am, 2013. 21(1): p. 1-19.

2. Molini, L., et al., Hip: Anatomy and US technique. J Ultrasound, 2011. 14(2): p. 99-108.

3. Piriou, P., et al., Can hip anatomy be reconstructed with femoral components having only one neck morphology? A study on 466 hips. J Arthroplasty, 2013. 28(7): p. 1185-91.

4. MD, J.R.H.F. Total HIp Replacement. OrthoInfo 20112011 [cited 2015; Available from: http://orthoinfo.aaos.org/topic.cfm?topic=a00377.

5. $\quad$ Netter, J.T.H.F.H., Chapter 5: Pelvis and Perineum, in Netter's Clinical Anatomy. 2010, Elsevier.

6. Bader, D.L., D.M. Salter, and T.T. Chowdhury, Biomechanical influence of cartilage homeostasis in health and disease. Arthritis, 2011. 2011: p. 979032.

7. Fitzgerald, J.B., et al., Mechanical compression of cartilage explants induces multiple time-dependent gene expression patterns and involves intracellular calcium and cyclic AMP. J Biol Chem, 2004. 279(19): p. 19502-11.

8. $\quad$ Clements, K.M., et al., How severe must repetitive loading be to kill chondrocytes in articular cartilage? Osteoarthritis Cartilage, 2001. 9(5): p. 499-507.

9. Buckwalter, J.A., C. Saltzman, and T. Brown, The impact of osteoarthritis: implications for research. Clin Orthop Relat Res, 2004(427 Suppl): p. S6-15.

10. Helmick, C.G., et al., Estimates of the prevalence of arthritis and other rheumatic conditions in the United States. Part I. Arthritis Rheum, 2008. 58(1): p. 15-25.

11. Lee, A.S., et al., A current review of molecular mechanisms regarding osteoarthritis and pain. Gene, 2013. 527(2): p. 440-7.

12. Hunter, D.J., J.J. McDougall, and F.J. Keefe, The symptoms of osteoarthritis and the genesis of pain. Med Clin North Am, 2009. 93(1): p. 83-100, xi.

13. Li, X., et al., Osteoarthritic tissues modulate functional properties of sensory neurons associated with symptomatic OA pain. Mol Biol Rep, 2011. 38(8): p. 5335-9. 
14. Leiro, J., et al., Effects of cis-resveratrol on inflammatory murine macrophages: antioxidant activity and down-regulation of inflammatory genes. J Leukoc Biol, 2004. 75(6): p. 1156-65.

15. Baker, E.N. and H.M. Baker, A structural framework for understanding the multifunctional character of lactoferrin. Biochimie, 2009. 91(1): p. 3-10.

16. Brand, C.A., et al., Chronic disease management: a review of current performance across quality of care domains and opportunities for improving osteoarthritis care. Rheum Dis Clin North Am, 2013. 39(1): p. 123-43.

17. Nelson, A.E., et al., A systematic review of recommendations and guidelines for the management of osteoarthritis: The chronic osteoarthritis management initiative of the U.S. bone and joint initiative. Semin Arthritis Rheum, 2014. 43(6): p. 701-12.

18. Rannou, F. and S. Poiraudeau, Non-pharmacological approaches for the treatment of osteoarthritis. Best Pract Res Clin Rheumatol, 2010. 24(1): p. 93106.

19. Lieberman, J.R., et al., Is Intra-Articular Hyaluronic Acid Effective in Treating Osteoarthritis of the Hip Joint? J Arthroplasty, 2015. 30(3): p. 507-511.

20. Lohmander, L.S. and E.M. Roos, Clinical update: treating osteoarthritis. Lancet, 2007. 370(9605): p. 2082-4.

21. Srinivasan, A., E. Jung, and B.R. Levine, Modularity of the femoral component in total hip arthroplasty. J Am Acad Orthop Surg, 2012. 20(4): p. 214-22.

22. Scheerlinck, T. and P.P. Casteleyn, The design features of cemented femoral hip implants. J Bone Joint Surg Br, 2006. 88(11): p. 1409-18.

23. Illgen, R., 2nd and H.E. Rubash, The optimal fixation of the cementless acetabular component in primary total hip arthroplasty. J Am Acad Orthop Surg, 2002. 10(1): p. 43-56.

24. Wassef, A.J. and T.P. Schmalzried, Femoral taperosis: an accident waiting to happen? Bone Joint J, 2013. 95-B(11 Suppl A): p. 3-6.

25. Soong, M., H.E. Rubash, and W. Macaulay, Dislocation after total hip arthroplasty. J Am Acad Orthop Surg, 2004. 12(5): p. 314-21.

26. Lemons, B.D.R.A.S.H.F.J.S.J.E., Biomaterials science: an introduction to materials in medicine. 1996: Academic Press. 
27. Valero Vidal, C., A. Olmo Juan, and A. Igual Munoz, Adsorption of bovine serum albumin on CoCrMo surface: effect of temperature and protein concentration. Colloids Surf B Biointerfaces, 2010. 80(1): p. 1-11.

28. T. Schmalzried, I.C., H. McKelop, Bearing Surfaces, in The Adult HIp, A.G.R.M. John J. Callaghan MD, Editor. 2006.

29. Mahoney, O.M. and J.H. Dimon, 3rd, Unsatisfactory results with a ceramic total hip prosthesis. J Bone Joint Surg Am, 1990. 72(5): p. 663-71.

30. Clarke, I.C. and A. Gustafson, Clinical and hip simulator comparisons of ceramic-on-polyethylene and metal-on-polyethylene wear. Clin Orthop Relat Res, 2000(379): p. 34-40.

31. McKellop, H., et al., Development of an extremely wear-resistant ultra high molecular weight polyethylene for total hip replacements. J Orthop Res, 1999. 17(2): p. 157-67.

32. Glyn-Jones, S., et al., The John Charnley Award: Highly crosslinked polyethylene in total hip arthroplasty decreases long-term wear: a double-blind randomized trial. Clin Orthop Relat Res, 2015. 473(2): p. 432-8.

33. Bozic, K.J., et al., The epidemiology of bearing surface usage in total hip arthroplasty in the United States. J Bone Joint Surg Am, 2009. 91(7): p. 1614-20.

34. Berry, D.J., et al., What are the current clinical issues in wear and tribocorrosion? Clin Orthop Relat Res, 2014. 472(12): p. 3659-64.

35. Babovic, N. and R.T. Trousdale, Total hip arthroplasty using highly cross-linked polyethylene in patients younger than 50 years with minimum 10-year follow-up. J Arthroplasty, 2013. 28(5): p. 815-7.

36. Jarrett, C.A., et al., The squeaking hip: a phenomenon of ceramic-on-ceramic total hip arthroplasty. J Bone Joint Surg Am, 2009. 91(6): p. 1344-9.

37. Andrews, R.E., et al., Effects of cobalt and chromium ions at clinically equivalent concentrations after metal-on-metal hip replacement on human osteoblasts and osteoclasts: implications for skeletal health. Bone, 2011. 49(4): p. 717-23.

38. National Joint Registry 10th Annual Report. 2013.

39. Annual Report 2011. 2011, Austrialia Orthopaedic Association: Australian Joint Registry.

40. Lehil, M.S. and K.J. Bozic, Trends in total hip arthroplasty implant utilization in the United States. J Arthroplasty, 2014. 29(10): p. 1915-8. 
41. Esposito, C.I., et al., What is the trouble with trunnions? Clin Orthop Relat Res, 2014. 472(12): p. 3652-8.

42. Collier, J.P., et al., Corrosion at the interface of cobalt-alloy heads on titaniumalloy stems. Clin Orthop Relat Res, 1991(271): p. 305-12.

43. Goldberg, J.R., et al., A multicenter retrieval study of the taper interfaces of modular hip prostheses. Clin Orthop Relat Res, 2002(401): p. 149-61.

44. Gill, I.P., et al., Corrosion at the neck-stem junction as a cause of metal ion release and pseudotumour formation. J Bone Joint Surg Br, 2012. 94(7): p. 895900.

45. Duwelius, P.J., et al., Clinical results of a modular neck hip system: hitting the "bull's-eye" more accurately. Am J Orthop (Belle Mead NJ), 2010. 39(10 Suppl): p. 2-6.

46. Kurtz, S.M., et al., Do ceramic femoral heads reduce taper fretting corrosion in hip arthroplasty? A retrieval study. Clin Orthop Relat Res, 2013. 471(10): p. 3270-82.

47. Cooper, H.J., et al., Corrosion at the head-neck taper as a cause for adverse local tissue reactions after total hip arthroplasty. J Bone Joint Surg Am, 2012. 94(18): p. 1655-61.

48. Haynes, E., Metal alloy, U.S.P. Office, Editor. 1907, Haynes, E.: USA.

49. Klarstrom, D., Wrought cobalt- base superalloys. Journal of Materials Engineering and Performance, 1993. 2(4): p. 523.

50. Temenoff, J.S.M.A.G., Biomaterials: The Intersection of Biology and Materials Science. 2008: Prentice Hall.

51. Kilner, T., et al., Phase identification and incipient melting in a cast Co--Cr surgical implant alloy. J Biomed Mater Res, 1982. 16(1): p. 63-79.

52. Scales, J.T., Journal of Bone and Joint Surgery, 1972. 53B.

53. Katti, K.S., Colloids and Surfaces B: Biointerfaces, 2004. 29.

54. Standard Specification for Cobalt-28 Chromium - 6 Molybdenum castings and casting alloy for surgical implants. ASTM International, 2012. 13. 
55. Standard Specification for Wrought Cobalt - 28Chromium - 6Molybdenum Alloys forSurgical Implants ASTM International, 2011.

56. Standard Specification for Cobalt-28Chromium-6Molybdenum Alloy Forgings for Surgical Implants>. ASTM International, 2011. 13.

57. López, H.F. and A.J. Saldivar-Garcia, Martensitic Transformation in a Cast CoCr-Mo-C Alloy. Metallurgical and Materials Transactions A, 2008. 39(1): p. 8.

58. Gupta, K., The Co-Cr-Mo (Cobalt-Chromium-Molybdenum) System. Journal of Phase Equilibria and Diffusion, 2005. 26(1): p. 87.

59. Wang, Q., L. Zhang, and J. Dong, Effects of Plasma Nitriding on Microstructure and Tribological Properties of CoCrMo Alloy Implant Materials. Journal of Bionic Engineering, 2010. 7(4): p. 337.

60. Caudillo, M., et al., On carbide dissolution in an as-cast ASTM F-75 alloy. Journal of Biomedical Materials Research, 2002. 59(2): p. 378.

61. Escobedo, J., et al., Effect of nitrogen on the microstructure and mechanical properties of a Co-Cr-Mo alloy. Materials and Design, 1996. 17(2): p. 79.

62. Bauer, S., et al., Engineering biocompatible implant surfaces:Part I: Materials and surfaces: Part I: Materials and surfaces. Progress in Materials Science, 2012.

63. Salinas-Rodrfguez, A. The Role of the FCC-HCP Phase Transformation During the Plastic Deformation of Co-Cr-Mo-C Alloys for Biomedical Applications,. in Cobalt-Base Alloys for Biomedical Applications, ASTM STP 1365. 1999. West Conshohocken, PA,: American Society for Testing and Materials,.

64. Nalbant, M., A. Altın, and H. Gökkaya, The effect of cutting speed and cutting tool geometry on machinability properties of nickel-base Inconel 718 super alloys. Materials \& Design, 2007. 28(4): p. 1334-1338.

65. Bordin, A., S. Bruschi, and A. Ghiotti, The Effect of Cutting Speed and Feed Rate on the Surface Integrity in Dry Turning of CoCrMo Alloy. Procedia CIRP, 2014. 13(0): p. 219-224.

66. Giacchi, J.V., et al., Microstructural characterization of as-cast biocompatible Co-Cr-Mo alloys. Materials Characterization, 2011. 62(1): p. 53-61.

67. Valero Vidal, C. and A. Igual Muñoz, Electrochemical characterisation of biomedical alloys for surgical implants in simulated body fluids. Corrosion Science, 2008. 50(7): p. 1954-1961. 
68. Salinas A.R., S.M.A., Lopez H.F., The effect of carbon on the mechanical strength of heat treated cast Co27Cr5Mo alloys, in The Minerals, Metals, and Materials Society. 1996: Warrendale, PA. p. 70-79.

69. Dobbs, H.S. and J.L.M. Robertson, Heat treatment of cast Co-Cr-Mo for orthopaedic implant use. Journal of Materials Science, 1983. 18(2): p. 391-401.

70. Rajan, K. and J. Vander Sande, Room temperature strengthening mechanisms in a Co-Cr-Mo-C alloy. Journal of Materials Science, 1982. 17(3): p. 769-778.

71. Montero-Ocampo, C., H. Lopez, and M. Talavera, Effect of alloy preheating on the mechanical properties of as-cast Co-Cr-Mo-C alloys. Metallurgical and Materials Transactions A, 1999. 30(3): p. 611-620.

72. Jacobs, J.J., et al., What do we know about taper corrosion in total hip arthroplasty? J Arthroplasty, 2014. 29(4): p. 668-9.

73. Fontana, M.G. and N.D. Greene, Chapter 1.3 Definition of Corrosion, in Corrosion engineering. 1978, McGraw-Hill: New York. p. 4-5.

74. McCafferty, E., What is Corrosion?, in Introduction to corrosion science. 2010, Springer: New York. p. 13-14.

75. Fontana, M.G. and N.D. Greene, Chapter 2.3 Electrochemical Reactions, in Corrosion Engineering. 1978, McGraw-Hill: New York. p. 14-17.

76. Ragone, D.V., 3.2 The Functions $F$ and $G$, in Thermodynamics of materials. 1995, Wiley: New York. p. 65-67.

77. McCafferty, E., The Free Eenrgy and Electrode Potential, in Introduction to corrosion science. 2010, Springer: New York. p. 65-67.

78. Latimer, W.M., The oxidation states of the elements and their potentials in aqueous solutions. $2 \mathrm{~d}$ ed. ed. Prentice-Hall chemistry series. 1952, New York: Prentice-Hall.

79. Fontana, M.G. and N.D. Greene, Chapter 9 Modern Theory - Principles, in Corrosion engineering. 1978, McGraw-Hill: New York. p. 456-462.

80. Fontana, M.G. and N.D. Greene, Chapter 4.13 Corrosion Testing. Standard Expressions for Corrosion Rate, in Corrosion engineering. 1978, McGraw-Hill: New York. p. 171-174.

81. Fontana, M.G. and N.D. Greene, Chapter 3: Eight Forms of Corrosion, in Corrosion engineering. 1978, McGraw-Hill: New York. p. 39-85. 
82. Virtanen, S., et al., Special modes of corrosion under physiological and simulated physiological conditions. Acta Biomater, 2008. 4(3): p. 468-76.

83. Konttinen, Y.T., et al., Acid attack and cathepsin $K$ in bone resorption around total hip replacement prosthesis. J Bone Miner Res, 2001. 16(10): p. 1780-6.

84. Rajan, K., Phase transformations in a wrought Co-Cr-Mo-C alloy. Metallurgical Transactions A, 1982. 13(7): p. 1161-1166.

85. Hodgson, A.W.E., et al., Passive and transpassive behaviour of CoCrMo in simulated biological solutions. Electrochimica Acta, 2004. 49(13): p. 2167-2178.

86. Li, Y.-S., et al., Surface-enhanced Raman spectroelectrochemical studies of corrosion films on implant Co-Cr-Mo alloy in biosimulating solutions. Journal of Raman Spectroscopy, 1999. 30(2): p. 97-103.

87. Muñoz, A.I. and S. Mischler, Interactive effects of albumin and phosphate ions on the corrosion of CoCrMo implant alloy. Journal of the electrochemical Society, 2007. 154(10): p. C562-C570.

88. Haeri, M., S. Goldberg, and J.L. Gilbert, The voltage-dependent electrochemical impedance spectroscopy of CoCrMo medical alloy using time-domain techniques: Generalized Cauchy-Lorentz, and KWW-Randles functions describing non-ideal interfacial behaviour. Corrosion Science, 2011. 53(2): p. 582-588.

89. Karimi, S., T. Nickchi, and A. Alfantazi, Effects of bovine serum albumin on the corrosion behaviour of AISI 316L, Co-28Cr-6Mo, and Ti-6Al-4V alloys in phosphate buffered saline solutions. Corrosion Science, 2011. 53(10): p. 32623272 .

90. Pound, B.G., Electrochemical behavior of cobalt-chromium alloys in a simulated physiological solution. Journal of Biomedical Materials Research Part A, 2010. 94A(1): p. 93-102.

91. Valero Vidal, C., A. Olmo Juan, and A. Igual Muñoz, Adsorption of bovine serum albumin on CoCrMo surface: Effect of temperature and protein concentration. Colloids and Surfaces B: Biointerfaces, 2010. 80(1): p. 1-11.

92. Kocijan, A., et al., Electrochemical Study of Co-Based Alloys in Simulated Physiological Solution. Journal of Applied Electrochemistry, 2004. 34(5): p. 517524.

93. Kocijan, A., I. Milošev, and B. Pihlar, Cobalt-based alloys for orthopaedic applications studied by electrochemical and XPS analysis. Journal of Materials Science: Materials in Medicine, 2004. 15(6): p. 643-650. 
94. Metikoš-Huković, M., et al., Influence of alloying elements on the corrosion stability of CoCrMo implant alloy in Hank's solution. Acta Biomaterialia, 2006. 2(6): p. 693-700.

95. Milošev, I. and H.H. Strehblow, The composition of the surface passive film formed on CoCrMo alloy in simulated physiological solution. Electrochimica Acta, 2003. 48(19): p. 2767-2774.

96. Ouerd, A., et al., Reactivity of CoCrMo alloy in physiological medium: Electrochemical characterization of the metal/protein interface. Electrochimica Acta, 2008. 53(13): p. 4461-4469.

97. Contu, F., B. Elsener, and H. Böhni, Electrochemical behavior of CoCrMo alloy in the active state in acidic and alkaline buffered solutions. Journal of the Electrochemical Society, 2003. 150(9): p. B419-B424.

98. Sargeant, A. and T. Goswami, Hip implants - Paper VI - Ion concentrations. Materials \& Design, 2007. 28(1): p. 155-171.

99. Yan, Y., A. Neville, and D. Dowson, Biotribocorrosion of CoCrMo orthopaedic implant materials-Assessing the formation and effect of the biofilm. Tribology International, 2007. 40(10-12): p. 1492-1499.

100. Body Fluids. 2013 [cited 2015; Available from: http://anatomyandphysiologyi.com/wp-content/uploads/2013/11/electrolytecomposition.jpg.

101. Kaiser, R., D.J. Browne, and K. Williamson, Investigation of the effects of cooling rate on the microstructure of investment cast biomedical grade Co alloys. IOP Conference Series: Materials Science and Engineering, 2012. 27(1): p. 012071.

102. Mineta, S., et al., Carbide Formation and Dissolution in Biomedical Co-Cr-Mo Alloys with Different Carbon Contents during Solution Treatment. Metallurgical and Materials Transactions A, 2010. 41(8): p. 2129-2138.

103. Kaiser, R., et al., The influence of cooling conditions on grain size, secondary phase precipitates and mechanical properties of biomedical alloy specimens produced by investment casting. Journal of the Mechanical Behavior of Biomedical Materials, 2013. 24(0): p. 53-63.

104. Turrubiates-Estrada, R., A. Salinas-Rodriguez, and H.F. Lopez, FCC to HCP transformation kinetics in a Co-27Cr-5Mo-0.23C alloy. Journal of Materials Science, 2011. 46(1): p. 254-262. 
105. Milošev, I., The effect of biomolecules on the behaviour of CoCrMo alloy in various simulated physiological solutions. Electrochimica Acta, 2012. 78(0): p. 259-273.

106. Valero Vidal, C. and A. Igual Muñoz, Study of the adsorption process of bovine serum albumin on passivated surfaces of CoCrMo biomedical alloy. Electrochimica Acta, 2010. 55(28): p. 8445-8452. 